\title{
Chirality transfer from the oxidative dearomatization of axially chiral binols with Oxone under mild conditions
}

\author{
Antonio Urbano*a,b Sara Vallejo, ${ }^{\mathrm{a}}$ María J. Cabrera-Afonso, ${ }^{\mathrm{a}}$ Elena Yonte ${ }^{\mathrm{a}}$ \\ aDepartamento de Química Orgánica, Universidad Autónoma de Madrid (UAM), 28049-Madrid, Spain. \\ ' Institute for Advanced Research in Chemical Sciences (IAdChem), UAM, 28049-Madrid, Spain. \\ antonio.urbano@uam.es
}

\section{Experimental Procedures}

General. All starting materials were purchased from commercial sources and used without further purification. Solvents used for reactions, extractions and purifications were reagent grade and used as received. When necessary, solvents were dried under standard conditions. Reactions were monitored by thin layer chromathography (TLC) using TLC silicagel coated aluminium plates $60 F_{254}$ (Merck) and visualized by ultraviolet light lamp $(254 \mathrm{~nm})$ and by staining with phosphomolybdic acid, followed by heating. Flash chromatography was performed with silicagel $60(0.040-0.063 \mathrm{~mm})$, packed with the corresponding eluent and run under positive air pressure.

Instrumentation. ${ }^{1} \mathrm{H}-\mathrm{NMR}$ and ${ }^{13} \mathrm{C}-\mathrm{NMR}$ spectra were performed on Bruker, Advance 300 (300 MHz for ${ }^{1} \mathrm{H}$ and $75 \mathrm{MHz}$ for ${ }^{13} \mathrm{C}$ ). Chemical shifts are expressed in ppm using the residual non-deuterated solvent as internal standard ( $\left.\mathrm{CDCl}_{3},{ }^{1} \mathrm{H}-\mathrm{NMR}: \delta 7.26 \mathrm{ppm},{ }^{13} \mathrm{C}-\mathrm{NMR}: \delta 77.16 \mathrm{ppm}\right)$. The abbreviations used for the multiplicities are s (singlet), d (doublet), dd (doublet of doublets), $t$ (triplet), td (triplet of doublets), $t t$ (triplet of triplets) and $\mathrm{m}$ (multiplet); and the coupling constants $(J)$ are reported in $\mathrm{Hertz}(\mathrm{Hz})$. Enantiomeric excesses were measured in a HPLC Agilent technologies, 1200 series. The HRMS measurements were performed using a hybrid quadrupole-time of flight (QTOF) mass analyzer. The slow addition of aqueous solution of Oxone was performed using a syringe pump Chemyx Fusion 100. Specific rotations were determined at $\mathrm{rt}$ on a Perkin Elmer $241 \mathrm{MC}$ polarimeter with sodium lamp $(\lambda: 589 \mathrm{~nm})$ in a $10 \mathrm{~cm}$ glass tube. Circular dichroism spectra were measured in $\mathrm{CH}_{2} \mathrm{Cl}_{2}$ in a quarzt cuvette $(1 \mathrm{~cm})$ at 298 o $\mathrm{K}$, using a JASCO J-815 CD spectropolarimeter. 


\section{Synthesis of substituted binols}

(R)-6,6'-Dibromo-[1,1'-binaphthalene]-2,2'-diol (1b)<smiles>Oc1ccc2cc(Br)ccc2c1-c1c(O)ccc2cc(Br)ccc12</smiles>

Compound $(R)$-1b was obtained from commercially available binol $(R)-1 \mathbf{a}(e e>99 \%)$ following a reported method described in the literature. ${ }^{1}$

${ }^{1} \mathrm{H}$ NMR $\left(300 \mathrm{MHz}, \mathrm{CDCl}_{3}\right)$ 8: $8.06(\mathrm{bs}, 2 \mathrm{H}), 7.90(\mathrm{~d}, J=9.0 \mathrm{~Hz}, 2 \mathrm{H}), 7.43-7.34(\mathrm{~m}, 4 \mathrm{H}), 6.98(\mathrm{~d}, J=8.9 \mathrm{~Hz}$, $2 \mathrm{H}), 5.01(\mathrm{~s}, 2 \mathrm{H})$.

Chiral HPLC: Daicel Chiralpak IC, n-hexane / 2-propanol: 90/10, flow rate: $0.5 \mathrm{~mL} / \mathrm{min}, \lambda: 254 \mathrm{~nm}, \mathrm{t}_{\mathrm{R}}: 10.88$ $\min , \mathrm{T}: 25^{\circ} \mathrm{C}, \boldsymbol{e e}>\mathbf{9 9 \%}$.
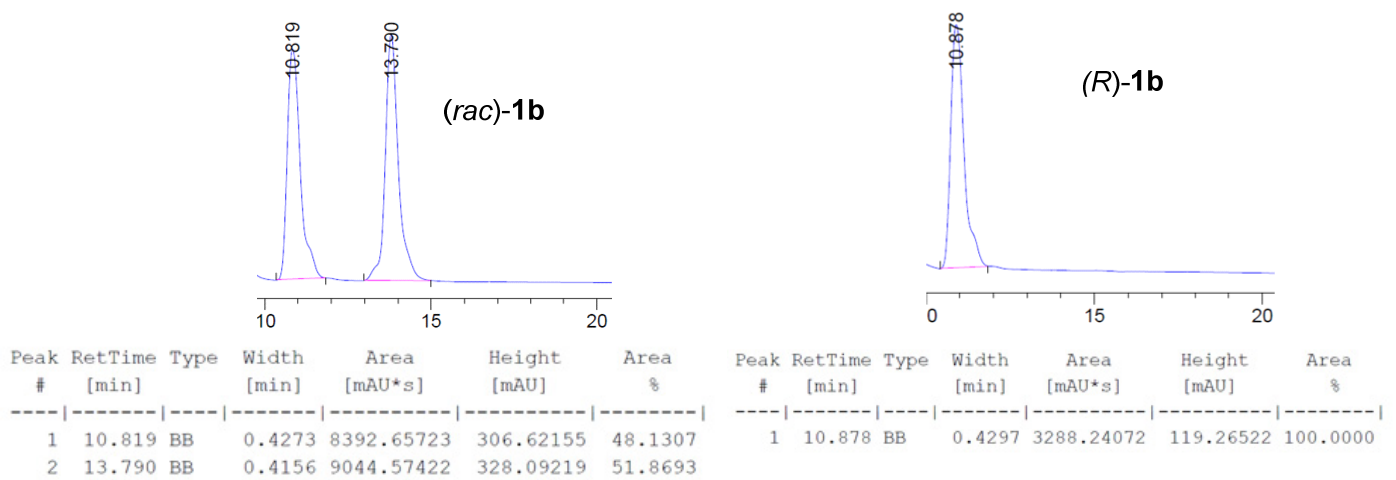

(R)-6,6'-Dimethyl-[1,1'-binaphthalene]-2,2'-diol (1c)<smiles>Cc1ccc2c(-c3c(O)ccc4cc(C)ccc34)c(O)ccc2c1</smiles>

${ }^{1}$ J. E. Beaver, B. C. Peacor, J. V. Bain, L. I. James, M. L. Waters, Org. Biomol. Chem. 2015, 13, 3220. 
To a mixture of binol (R)-1b (ee >99\%) (150 mg, $0.34 \mathrm{mmol}$ ) and $\mathrm{PdCl}_{2}(\mathrm{dppf}) \cdot \mathrm{CH}_{2} \mathrm{Cl}_{2}(55.4 \mathrm{mg}, 0.07 \mathrm{mmol}, 20$ $\mathrm{mol} \%)$ in dry THF $(3.4 \mathrm{~mL})$ at $0 \stackrel{\circ}{ }{ }^{\circ}$, under nitrogen, methylmagnesium bromide $(0.39 \mathrm{~mL}, 3.39 \mathrm{mmol})$ was added. The reaction mixture was refluxed for $17 \mathrm{~h}$ in an oil bath, cooled to rt, quenched with sat. $\mathrm{NH}_{4} \mathrm{Cl}$ (aq.) and extracted with EtOAc (x3). The combined organic layers were washed with sat. $\mathrm{NH}_{4} \mathrm{Cl}$ (aq.), $\mathrm{NaHCO}_{3}$ (aq.), brine, dried over $\mathrm{Na}_{2} \mathrm{SO}_{4}$ and concentrated under reduced pressure. The crude was purified by flash chromatography $\left(\mathrm{CH}_{2} \mathrm{Cl}_{2} / \mathrm{EtOAc} 80: 1\right)$ to give binol $(R)-1 \mathrm{c}$ in $99 \%$ yield $(105.5 \mathrm{mg}, 0.34 \mathrm{mmol})$, as a white solid. Spectral data were in agreement with those reported in the literature. ${ }^{2}$

${ }^{1} \mathrm{H} \mathrm{NMR}\left(300 \mathrm{MHz}, \mathrm{CDCl}_{3}\right)$ 8: $7.86(\mathrm{~d}, J=8.9 \mathrm{~Hz}, 2 \mathrm{H}), 7.68(\mathrm{bs}, 2 \mathrm{H}), 7.33(\mathrm{~d}, J=8.9 \mathrm{~Hz}, 2 \mathrm{H}), 7.17(\mathrm{dd}, J=8.6,1.6 \mathrm{~Hz}$, $2 \mathrm{H}), 7.08(\mathrm{~d}, J=8.6 \mathrm{~Hz}, 2 \mathrm{H}), 5.03(\mathrm{~s}, 2 \mathrm{H}), 2.50(\mathrm{~s}, 6 \mathrm{H})$.

Chiral HPLC: Daicel Chiralpak IC, n-hexane / 2-propanol: 90/10, flow rate: $1.0 \mathrm{~mL} / \mathrm{min}, \lambda: 254 \mathrm{~nm}, \mathrm{t}_{\mathrm{R}}$ (major): 8.45 $\min , t_{R}$ (minor): $11.78 \min T: 25 \stackrel{\circ}{C}, 99 \%$ ee.
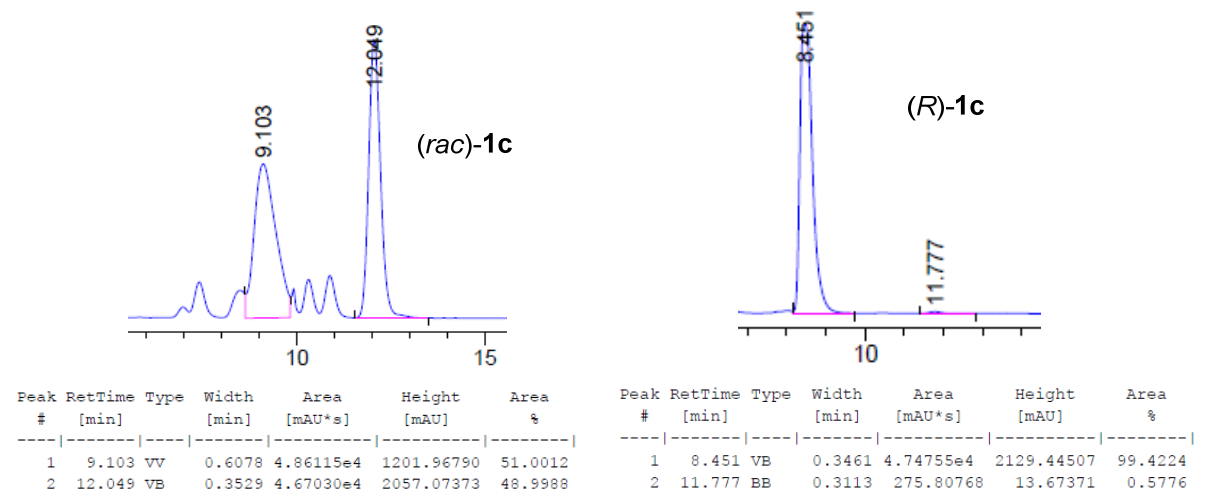

\section{(R)-6,6'-Diethyl-[1,1'-binaphthalene]-2,2'-diol (1d)}<smiles>CCc1ccc2c(-c3c(O)ccc4cc(Br)ccc34)c(CC)ccc2c1</smiles>

To a solution of binol $(R)-\mathbf{1 b}$ (ee $>99 \%)(150.0 \mathrm{mg}, 0.34 \mathrm{mmol})$ and $\mathrm{PdCl}_{2}(\mathrm{dppf}) \cdot \mathrm{CH}_{2} \mathrm{Cl}_{2}(55.4 \mathrm{mg}, 0.07 \mathrm{mmol}$, $20 \mathrm{~mol} \%)$ in dry THF (3.4 mL) at $0{ }^{\circ} \mathrm{C}$, under nitrogen, ethylmagnesium bromide $(0.89 \mathrm{~mL}, 6.79 \mathrm{mmol})$ was

\footnotetext{
${ }^{2}$ Verga, D.; Percivalle, C.; Doria, F.; Porta, A.; Freccero, M. J. Org. Chem. 2011, 76, 2319.
} 
added. The reaction mixture was refluxed for $21 \mathrm{~h}$ in an oil bath, cooled to rt, quenched wit sat. $\mathrm{NH}_{4} \mathrm{Cl}$ (aq.) and extracted with ethyl acetate (x3). The organic layer was washed with sat. $\mathrm{NH}_{4} \mathrm{Cl}$ (aq.), sat. $\mathrm{NaHCO}_{3}$ (aq.), brine and dried over $\mathrm{MgSO}_{4}$ and concentrated under reduced pressure. The crude was purified by flash chromatography (heptane/EtOAc 10:1) to give binol ( $R$ )-1d in $82 \%$ yield (100.7 mg, $0.28 \mathrm{mmol}$ ), as a pale yellow solid.

${ }^{1} \mathrm{H}$ NMR $\left(300 \mathrm{MHz}, \mathrm{CDCl}_{3}\right)$ 8: 7.90 (d, $\left.J=8.9 \mathrm{~Hz}, 2 \mathrm{H}\right), 7.68$ (bs, 2H), 7.35 (d, $\left.J=8.9 \mathrm{~Hz}, 2 \mathrm{H}\right), 7.19$ (dd, $J=$ 8.6, $1.8 \mathrm{~Hz}, 2 \mathrm{H}), 7.10(\mathrm{~d}, J=8.6 \mathrm{~Hz}, 2 \mathrm{H}), 4.99(\mathrm{~s}, 2 \mathrm{H}), 2.78(\mathrm{q}, J=7.6 \mathrm{~Hz}, 4 \mathrm{H}), 1.31(\mathrm{t}, J=7.6 \mathrm{~Hz}, 6 \mathrm{H})$.

${ }^{13} \mathrm{C} \mathrm{NMR}\left(75 \mathrm{MHz}, \mathrm{CDCl}_{3}\right)$ 8: 152.2, 140.0, 131.9, 130.9, 129.8, 128.7, 126.3, 124.4, 117.8, 111.1, 28.8, 15.6.

HRMS (ESI): calculated for $\mathrm{C}_{24} \mathrm{H}_{22} \mathrm{NaO}_{2}\left([\mathrm{M}+\mathrm{Na}]^{+}\right) 365.1512$, found 365.1517 .

$[\alpha]_{\mathrm{D}}{ }^{20}=-45.0\left(c=0.02, \mathrm{CHCl}_{3}\right)$.

Chiral HPLC: Daicel Chiralpak IC, $n$-hexane / 2-propanol: 90/10, flow rate: $1.0 \mathrm{~mL} / \mathrm{min}, \lambda: 254 \mathrm{~nm}, t_{R}$ (major): 7.41 $\min , t_{R}$ (minor): $10.67 \min , \mathrm{T}: 25^{\circ} \mathrm{C}, 95 \%$ ee.

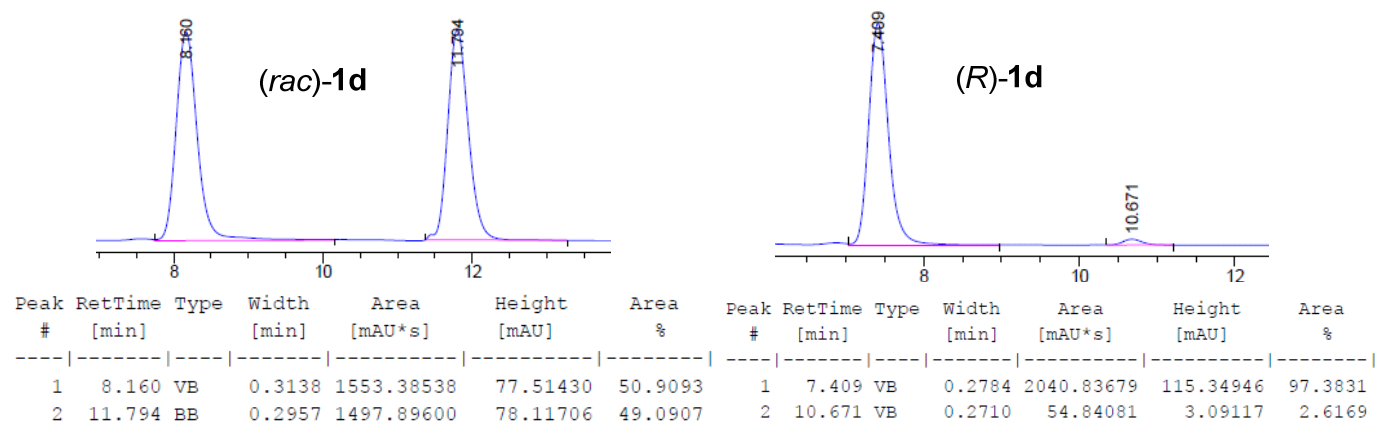

(R)-6,6'-Dihexyl-[1,1'-binaphthalene]-2,2'-diol (1e)<smiles>CCCCCCc1ccc2c(-c3c(O)ccc4cc(CCCCCC)ccc34)c(O)ccc2c1</smiles>

To a solution of binol $(R)-\mathbf{1 b}$ (ee $>99 \%)(100.0 \mathrm{mg}, 0.22 \mathrm{mmol})$ and $\mathrm{PdCl}_{2}(\mathrm{dppf}) \cdot \mathrm{CH}_{2} \mathrm{Cl}_{2}(55.4 \mathrm{mg}, 0.07 \mathrm{mmol}$, $20 \mathrm{~mol} \%)$ in dry THF (2.26 mL) at $0 \stackrel{\circ}{ }{ }^{\circ}$, under nitrogen, hexylmagnesium bromide $(0.88 \mathrm{~mL}, 4.53 \mathrm{mmol})$ was added. The reaction mixture was refluxed for $21 \mathrm{~h}$ in an oil bath, cooled to rt, quenched wit sat. $\mathrm{NH}_{4} \mathrm{Cl}$ (aq.) and 
extracted with ethyl acetate (x3). The organic layer was washed with sat. $\mathrm{NH}_{4} \mathrm{Cl}$ (aq.), sat. $\mathrm{NaHCO}_{3}$ (aq.), brine and dried over $\mathrm{MgSO}_{4}$ and concentrated to dryness under reduced pressure. The crude was purified by flash chromatography (heptane/EtOAc 8:1) to give binol (R)-1e in $95 \%$ yield ( $97.8 \mathrm{mg}, 0.22 \mathrm{mmol}$ ), as a white solid.

${ }^{1} \mathrm{H} \mathrm{NMR}\left(300 \mathrm{MHz}, \mathrm{CDCl}_{3}\right)$ 8: $7.88(\mathrm{~d}, J=8.9 \mathrm{~Hz}, 2 \mathrm{H}), 7.67(\mathrm{~s}, 2 \mathrm{H}), 7.34(\mathrm{~d}, J=8.9 \mathrm{~Hz}, 2 \mathrm{H}), 7.19(\mathrm{dd}, J=8.6$, $1.7 \mathrm{~Hz}, 2 \mathrm{H}), 7.12(\mathrm{~d}, J=8.6 \mathrm{~Hz}, 2 \mathrm{H}), 5.06(\mathrm{~s}, 2 \mathrm{H}), 2.76(\mathrm{t}, J=7.8 \mathrm{~Hz}, 4 \mathrm{H}), 1.80-1.63(\mathrm{~m}, 4 \mathrm{H}), 1.46-1.30(\mathrm{~m}$, $12 \mathrm{H}), 0.98-0.90(\mathrm{~m}, 6 \mathrm{H})$.

${ }^{13} \mathrm{C} \mathrm{NMR}\left(75 \mathrm{MHz}, \mathrm{CDCl}_{3}\right)$ 8: 152.2, 138.7, 131.9, 130.8, 129.7, 129.1, 126.9, 124.3, 117.8, 111.1, 35.9, 31.9, $31.5,29.2,22.8,14.2$.

HRMS (ESI): calculated for $\mathrm{C}_{32} \mathrm{H}_{38} \mathrm{NaO}_{2}\left([\mathrm{M}+\mathrm{Na}]^{+}\right)$477.2762, found 477.2783 .

$[\alpha]_{D}{ }^{20}=-29.2\left(c=0.024, \mathrm{CHCl}_{3}\right)$.

Chiral HPLC: Daicel Chiralpak IC, $n$-hexane / 2-propanol: 90/10, flow rate: $0.8 \mathrm{~mL} / \mathrm{min}, \lambda: 254 \mathrm{~nm}, t_{R}$ (major): 6.97 $\min , t_{R}$ (minor): $9.74 \mathrm{~min}, \mathrm{~T}: 25^{\circ} \mathrm{C}, 97 \%$ ee.

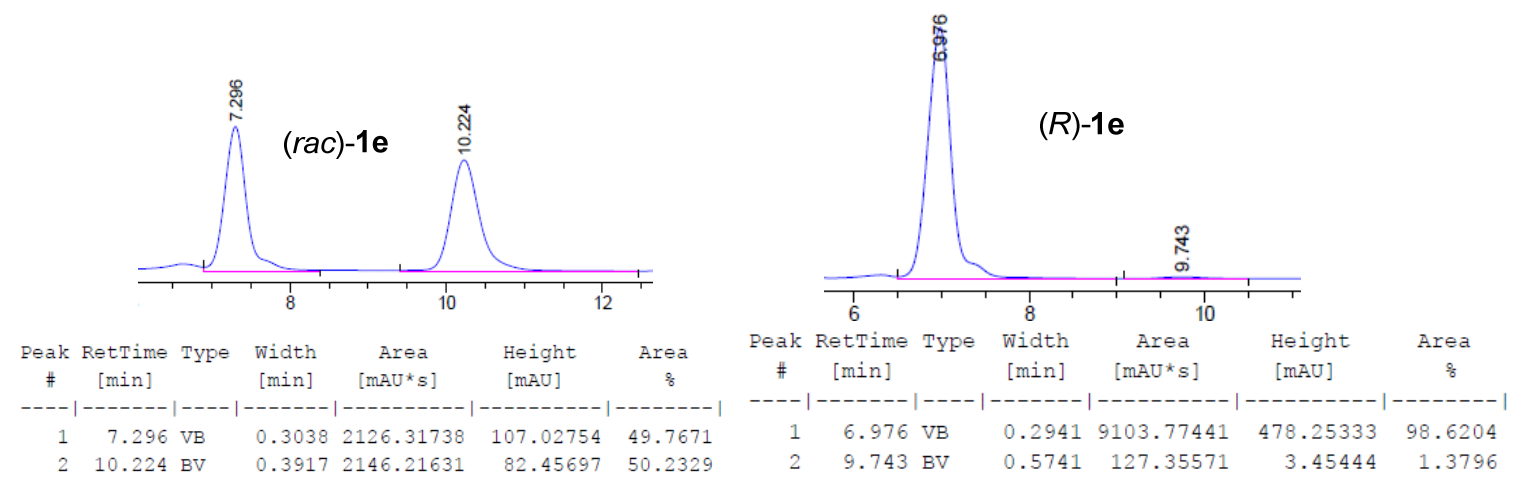

(rac)-6,6'-Diisopropyl-[1,1'-binaphthalene]-2,2'-diol (1f)<smiles>CC(C)c1ccc2c(-c3c(O)ccc4cc(C(C)C)ccc34)c(O)ccc2c1</smiles> 
To a solution of binol (rac)-1b (150.0 mg, $0.34 \mathrm{mmol})$ and $\mathrm{PdCl}_{2}(\mathrm{dppf}) \cdot \mathrm{CH}_{2} \mathrm{Cl}_{2}(55.4 \mathrm{mg}, 0.07 \mathrm{mmol}, 20 \mathrm{~mol} \%)$ in dry THF (3.4 mL) at $0 \stackrel{\circ}{ } \mathrm{C}$, under nitrogen, isopropylmagnesium bromide $(1.2 \mathrm{~mL}, 6.8 \mathrm{mmol})$ was added. The reaction mixture was refluxed for $22 \mathrm{~h}$ in an oil bath, cooled to rt, quenched wit sat. $\mathrm{NH}_{4} \mathrm{Cl}$ (aq.) and extracted with ethyl acetate (x3). The organic layer was washed with sat. $\mathrm{NH}_{4} \mathrm{Cl}$ (aq.), sat. $\mathrm{NaHCO}_{3}$ (aq.), brine and dried over $\mathrm{MgSO}_{4}$ and concentrated to dryness under reduced pressure. The crude was purified by flash chromatography (heptane/EtOAc 6:1) to give binol (rac)-1f in $81 \%$ yield ( $84.9 \mathrm{mg}, 0.28 \mathrm{mmol}$ ), as a white solid. Spectral data were in agreement with those reported in the literature. ${ }^{3}$

${ }^{1} \mathrm{H}$ NMR (300 MHz, CDCl $)_{3}$ 8: $7.91(\mathrm{~d}, J=8.9 \mathrm{~Hz}, 2 \mathrm{H}), 7.71(\mathrm{~d}, J=1.7 \mathrm{~Hz}, 2 \mathrm{H}), 7.35(\mathrm{~d}, J=8.9 \mathrm{~Hz}, 2 \mathrm{H}), 7.24$ (dd, $J=8.7,1.8 \mathrm{~Hz}, 2 \mathrm{H}$ ), $7.14(\mathrm{~d}, J=8.6 \mathrm{~Hz}, 2 \mathrm{H}$ ), $5.01(\mathrm{~s}, 2 \mathrm{H}), 3.05$ (hept, $J=6.9 \mathrm{~Hz}, 2 \mathrm{H}$ ), 1.34 (dd, $J=6.9$, $1.9 \mathrm{~Hz}, 12 \mathrm{H})$.

(R)-6,6'-Di-tert-butyl-[1,1'-binaphthalene]-2,2'-diol (1g)<smiles>CC(C)(C)c1ccc2c(-c3c(O)ccc4cc(C(C)(C)C)ccc34)c(O)ccc2c1</smiles>

Compound $(R)-\mathbf{1 g}$ was obtained from commercially available binol $(R)-1 \mathrm{a}$ (ee $>99 \%)$ following a reported method described in the literature. ${ }^{4}$

${ }^{1} \mathrm{H} \mathrm{NMR}\left(300 \mathrm{MHz}, \mathrm{CDCl}_{3}\right) \delta: 7.94(\mathrm{~d}, J=8.9 \mathrm{~Hz}, 2 \mathrm{H}), 7.82(\mathrm{~d}, J=2.0 \mathrm{~Hz}, 2 \mathrm{H}), 7.41(\mathrm{dd}, J=8.8,2.1 \mathrm{~Hz}, 2 \mathrm{H}), 7.36(\mathrm{~d}$, $J=8.9 \mathrm{~Hz}, 2 \mathrm{H}), 7.14(\mathrm{~d}, J=8.8 \mathrm{~Hz}, 2 \mathrm{H}), 4.97(\mathrm{~s}, 2 \mathrm{H}), 1.39(\mathrm{~s}, 18 \mathrm{H})$.

Chiral HPLC: Daicel Chiralpak IC, n-hexane / 2-propanol: 95/5, flow rate: $1.0 \mathrm{~mL} / \mathrm{min}, \lambda=254 \mathrm{~nm}, \mathrm{t}_{\mathrm{R}}$ (major): 7.99 $\min , t_{R}$ (minor): $13.66 \min T: 25 \stackrel{\circ}{\circ}, 97 \%$ ee.

\footnotetext{
${ }^{3}$ S. Narute, R. Parnes, F. D. Toste, D. Pappo, J. Am. Chem. Soc. 2016, 138, 16553.

${ }^{4}$ E. Balaraman, K. C. Kumara Swamy, Tetrahedron: Asymmetry. 2007, 18, 2037.
} 

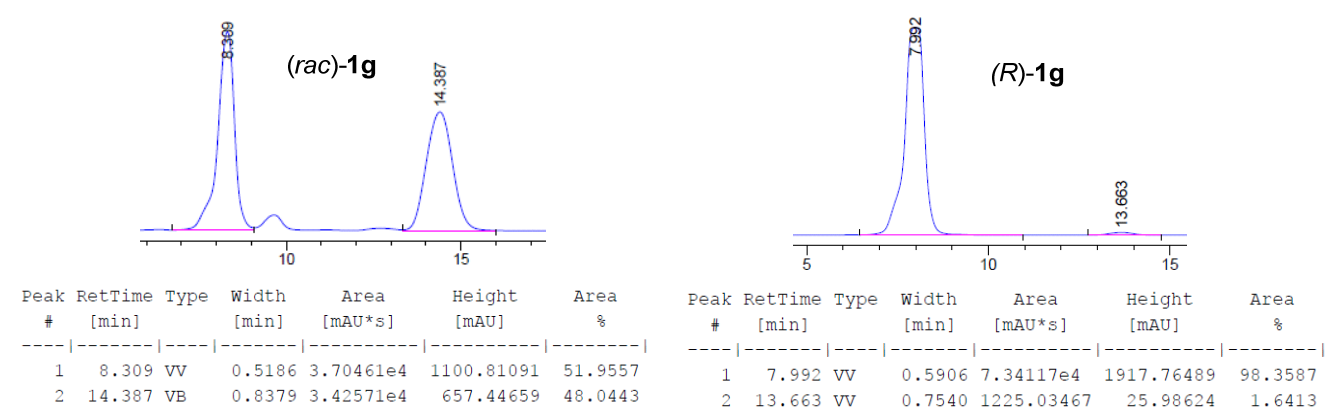

\section{(R)-6,6'-Di(1-adamantyl)-[1,1'-binaphthalene]-2,2'-diol (1h)}

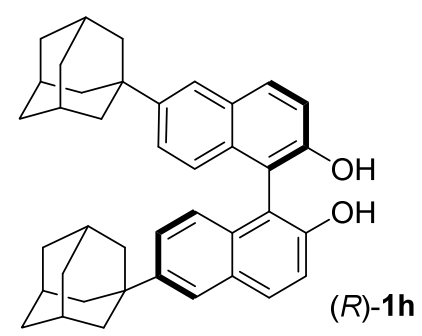

Compound $(R)$-1h was obtained from commercially available binol $(R)-1$ a $(e e>99 \%)$ following a reported method described in the literature. ${ }^{5}$

${ }^{1} \mathrm{H} \mathrm{NMR}\left(300 \mathrm{MHz}, \mathrm{CDCl}_{3}\right)$ 8: $7.94(\mathrm{~d}, J=8.9 \mathrm{~Hz}, 2 \mathrm{H}), 7.78(\mathrm{bs}, 2 \mathrm{H}), 7.37-7.25(\mathrm{~m}, 4 \mathrm{H}), 7.10(\mathrm{~d}, J=8.9 \mathrm{~Hz}$, $2 \mathrm{H}), 5.00(\mathrm{bs}, 2 \mathrm{H}), 2.12(\mathrm{~m}, 6 \mathrm{H}), 2.02-1.90(\mathrm{~m}, 12 \mathrm{H}), 1.88-1.73(\mathrm{~m}, 12 \mathrm{H})$.

Chiral HPLC: Daicel Chiralpak IC, n-hexane / 2-propanol: 90/10, flow rate: $1.0 \mathrm{~mL} / \mathrm{min}, \lambda: 230 \mathrm{~nm}, \mathrm{t}_{\mathrm{R}}$ (major): 7.00 $\min , t_{R}$ (minor): $11.48 \min T: 25 \stackrel{\circ}{ }$, $99 \%$ ee.

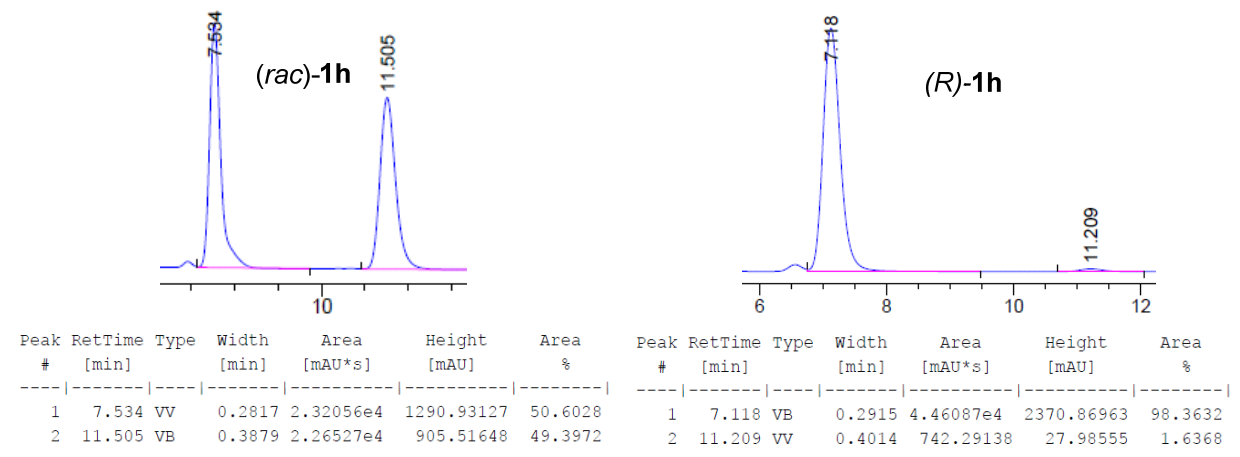

${ }^{5}$ R. Navarro, C. Monterde, M. Iglesias, F. Sánchez, ACS Omega. 2018, 3, 1197. 


\section{(R)-6,6'-Diphenyl-[1,1'-binaphthalene]-2,2'-diol (1i)}<smiles>Oc1ccc2cc(-c3ccccc3)ccc2c1-c1c(O)ccc2cc(-c3ccccc3)ccc12</smiles>

In a dried sealed tube, binol ( $R$ )-1b (ee >99\%) $(101.0 \mathrm{mg}, 0.22 \mathrm{mmol})$, phenylboronic acid ( $68.6 \mathrm{mg}, 0.56 \mathrm{mmol})$, $\mathrm{K}_{2} \mathrm{CO}_{3}(76.0 \mathrm{mg}, 0.55 \mathrm{mmol})$ and $\mathrm{Pd}\left(\mathrm{PPh}_{3}\right)_{4}(12.7 \mathrm{mg}, 0.03 \mathrm{mmol}, 5 \mathrm{~mol} \%)$ were dissolved in a mixture of deoxygenated THF $(0.69 \mathrm{~mL})$ and water $(0.65 \mathrm{~mL})$. The mixture was refluxed for $16 \mathrm{~h}$ in an oil bath and cooled to $r$ t. The organic layer was separated and the aqueous layer was extracted with EtOAc (x3). The combined organic layers were dried over $\mathrm{Na}_{2} \mathrm{SO}_{4}$ and concentrated to dryness under reduced pressure. The crude was purified by flash chromatography (heptane/EtOAc 5:1) to give binol ( $R$ )-1i in $79 \%$ yield ( $78.6 \mathrm{mg}, 0.18 \mathrm{mmol}$ ), as a white solid. Spectral data were in agreement with those reported in the literature. ${ }^{6}$

${ }^{1} \mathrm{H}$ NMR $\left(300 \mathrm{MHz}, \mathrm{CDCl}_{3}\right)$ 8: $8.12(\mathrm{~d}, J=1.9 \mathrm{~Hz}, 2 \mathrm{H}), 8.05(\mathrm{~d}, J=8.9 \mathrm{~Hz}, 2 \mathrm{H}), 7.74-7.64(\mathrm{~m}, 4 \mathrm{H}), 7.61(\mathrm{dd}$, $J=8.7,1.9 \mathrm{~Hz}, 2 \mathrm{H}), 7.54-7.41(\mathrm{~m}, 6 \mathrm{H}), 7.41-7.35(\mathrm{~m}, 2 \mathrm{H}), 7.28(\mathrm{~d}, J=8.8 \mathrm{~Hz}, 2 \mathrm{H}), 5.13(\mathrm{~s}, 2 \mathrm{H})$.

Chiral HPLC: Daicel Chiralpak IC, $n$-hexane / 2-propanol: 90/10, flow rate: $1.0 \mathrm{~mL} / \mathrm{min}, \lambda: 254 \mathrm{~nm}, t_{R}: 8.79 \mathrm{~min}, \mathrm{~T}$ : $25^{\circ} \mathrm{C},>\mathbf{9 9 \%}$ ee.
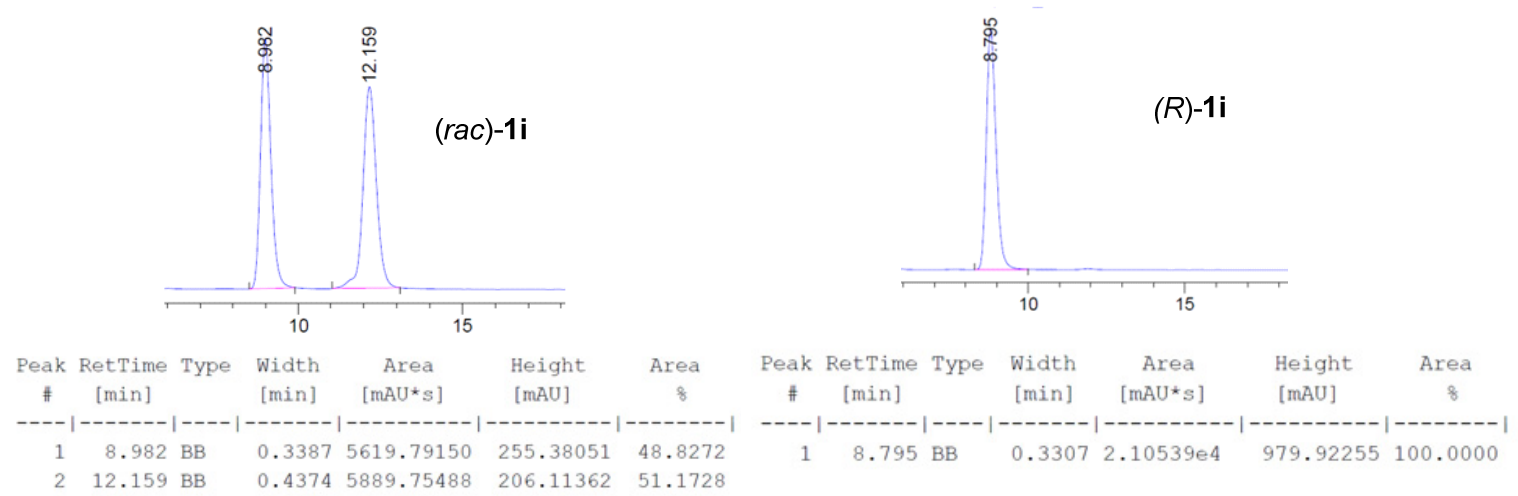

${ }^{6}$ G. Kumaraswamy, M. N. V. Sastry, N. Jena, K. R. Kumar, M. Vairamani, Tetrahedron: Asymmetry. 2003, $14,3797$. 


\section{(R)-6,6'-Bis(3,5-bis(trifluoromethyl)phenyl)-[1,1'-binaphthalene]-2,2'-diol (1j)}<smiles>OC1=Cc2cc(-c3cc(C(F)(F)F)cc(C(F)(F)F)c3)ccc2C2=c3ccc(-c4cc(C(F)(F)F)cc(C(F)(F)F)c4)cc3=CC2=C(O)C=C1</smiles>

In a dried sealed tube, binol ( $R$ )-1b (ee >99\%) (200 mg, $0.45 \mathrm{mmol}),(3,5-$ bis(trifluoromethyl)phenyl)boronic acid (149.7 mg, $0.58 \mathrm{mmol}), \mathrm{K}_{2} \mathrm{CO}_{3}(156.4 \mathrm{mg}, 1.13 \mathrm{mmol})$ and $\mathrm{Pd}\left(\mathrm{PPh}_{3}\right)_{4}(26.2 \mathrm{mg}, 0.02 \mathrm{mmol}, 5 \mathrm{~mol} \%)$ were dissolved in a mixture of deoxygenated THF $(1.38 \mathrm{~mL})$ and water $(1.30 \mathrm{~mL})$. The mixture was refluxed for $24 \mathrm{~h}$ in an oil bath and cooled to rt. The organic layer was separated and the aqueous layer was extracted with EtOAc (x3). The combined organic layers were dried over $\mathrm{Na}_{2} \mathrm{SO}_{4}$ and concentrated to dryness under reduced pressure. The crude was purified by flash chromatography (heptane/EtOAc 3:1) to give binol ( $R$ )-1j in $80 \%$ yield ( $263.9 \mathrm{mg}, 0.36 \mathrm{mmol}$ ), as a white solid. Spectral data were in agreement with those reported in the literature. ${ }^{7}$

${ }^{1} \mathrm{H} \mathrm{NMR}\left(300 \mathrm{MHz}, \mathrm{CDCl}_{3}\right)$ 8: $8.15(\mathrm{~d}, J=1.9 \mathrm{~Hz}, 2 \mathrm{H}), 8.12(\mathrm{bs}, 2 \mathrm{H}), 8.09(\mathrm{~m}, 4 \mathrm{H}), 7.88(\mathrm{bs}, 2 \mathrm{H}), 7.56(\mathrm{dd}, J=$ 8.7, $2.0 \mathrm{~Hz}, 2 \mathrm{H}$ ), 7.48 (d, $J=8.9 \mathrm{~Hz}, 2 \mathrm{H}), 7.28(\mathrm{~d}, J=8.8 \mathrm{~Hz}, 2 \mathrm{H}), 5.17$ (bs, $2 \mathrm{H})$.

We were not able to evaluate the optical purity of $(R)-\mathbf{1} \mathbf{j}$ at this stage but the corresponding oxidative dearomatization product $(6 \mathrm{a} R, \mathbf{1 3} \mathrm{CS})-\mathbf{3} \mathbf{j}$ showed an $\boldsymbol{e e}>\mathbf{9 9 \%}$ (see page $\mathrm{S}-30$ ).

(rac)-6,6'-Bis(phenylethynyl)-[1,1'-binaphthalene]-2,2'-diol (1k)

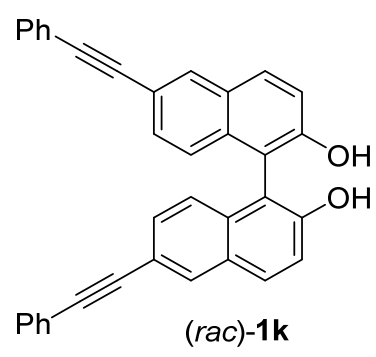

${ }^{7}$ R. Bisht, J. Chaturvedi, G. Pandey, B. Chattopadhyay, Org. Lett. 2019, 21, 6476. 
Compound ( $r a c)$-1k was obtained from binol ( $r a c)$-1b following a reported method described in the literature. $^{8}$

${ }^{1} \mathrm{H} \mathrm{NMR}\left(300 \mathrm{MHz}, \mathrm{CDCl}_{3}\right) \delta: 8.00(\mathrm{bs}, 2 \mathrm{H}), 7.83(\mathrm{~d}, J=9.0 \mathrm{~Hz}, 2 \mathrm{H}), 7.53-7.45(\mathrm{~m}, 4 \mathrm{H}), 7.38-7.22(\mathrm{~m}, 10 \mathrm{H}), 7.01$ $(\mathrm{d}, J=8.7 \mathrm{~Hz}, 2 \mathrm{H}), 5.10(\mathrm{bs}, 2 \mathrm{H})$.

(rac)-6,6'-Di(hex-1-yn-1-yl)-[1,1'-binaphthalene]-2,2'-diol (1I)

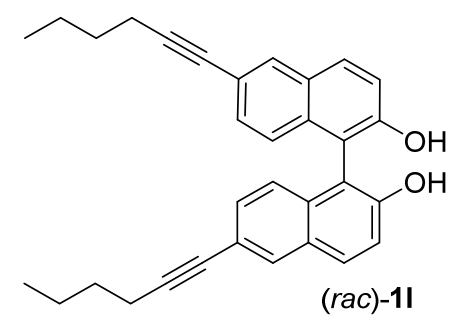

Compound (rac)-1l was obtained from binol (rac)-1b following a reported method described in the literature. $^{9}$

${ }^{1} \mathrm{H}$ NMR (300 MHz, CDCl $)_{3}$ ) $: 7.95$ (bs, 2H), $7.89(\mathrm{~d}, J=8.9 \mathrm{~Hz}, 2 \mathrm{H}), 7.36(\mathrm{~d}, J=8.9 \mathrm{~Hz}, 2 \mathrm{H}), 7.29$ (dd, $J=8.8$, $1.8 \mathrm{~Hz}, 2 \mathrm{H}), 7.03(\mathrm{~d}, J=8.7 \mathrm{~Hz}, 2 \mathrm{H}), 5.10(\mathrm{~s}, 2 \mathrm{H}), 2.45(\mathrm{t}, J=7.0 \mathrm{~Hz}, 4 \mathrm{H}), 1.70-1.56(\mathrm{~m}, 4 \mathrm{H}), 1.56-1.45(\mathrm{~m}$, $4 \mathrm{H}), 0.97(\mathrm{t}, J=7.2 \mathrm{~Hz}, 6 \mathrm{H})$.

(rac)-6,6'-Dinitro-[1,1'-binaphthalene]-2,2'-diol (1m)<smiles>Cc1ccc2c(-c3c([N+](=O)[O-])ccc4cc([N+](=O)[O-])ccc34)c(O)ccc2c1</smiles>

Compound ( $\mathrm{rac}$-1q was obtained following a reported method described in the literature. ${ }^{10} t$-Butyl nitrite ( $t$-Bu-ONO) (232 $\mu \mathrm{l}, 3.5 \mathrm{mmol})$ was added to a solution of commercially available binol (rac)-1a (143,2 mg,

\footnotetext{
${ }^{8}$ H. Sasai, T. Tokunaga, S. Watanabe, T. Suzuki, N. Itoh, M. Shibasaki, J. Org. Chem. 1995, 60, 7388.

${ }^{9}$ R. Zimmer, L. Schefzig, A. Peritz, V. Dekaris, H.-U. Reissig, Synthesis 2004, 1439.

${ }^{10}$ Q. Wang, G. Xu, M. Xian, C. Lv, Chinese patent 2018, CN 108658774 A 20181016.
} 
$0.5 \mathrm{mmol}$ ) in THF $(2.5 \mathrm{~mL})$. The reaction mixture was stirred, open to the air, at $\mathrm{rt}$ for $18 \mathrm{~h}$ and the solvent evaporated under reduced pressure. The residue was purified by flash chromatography $\left(\mathrm{CH}_{2} \mathrm{Cl}_{2} / \mathrm{EtOAc} 10: 1\right)$ to give binol (rac)-1m in $61 \%$ yield $(114.6 \mathrm{mg}, 0.30 \mathrm{mmol})$, as a yellow solid.

${ }^{1} \mathrm{H}$ NMR (300 MHz, acetone-d $)$ ): $9.01(\mathrm{~d}, J=2.4 \mathrm{~Hz}, 2 \mathrm{H}), 8.40(\mathrm{~d}, J=9.1 \mathrm{~Hz}, 2 \mathrm{H}), 8.12(\mathrm{dd}, J=9.3,2.4 \mathrm{~Hz}$, $2 \mathrm{H}), 7.67(\mathrm{~d}, J=9.0 \mathrm{~Hz}, 2 \mathrm{H}), 7.33(\mathrm{~d}, J=9.2 \mathrm{~Hz}, 2 \mathrm{H})$.

(rac)-7,7'-Dibromo-[1,1'-binaphthalene]-2,2'-diol (1n)

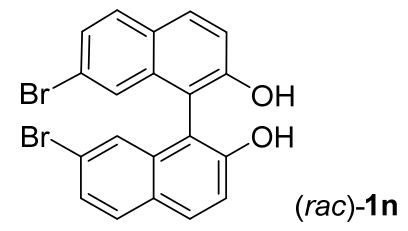

Compound ( $r a c)-1 \mathrm{n}$ was obtained following a reported method described in the literature. ${ }^{11}$

${ }^{1} \mathrm{H} \mathrm{NMR}\left(300 \mathrm{MHz}, \mathrm{CDCl}_{3}\right)$ 8: $7.93(\mathrm{~d}, J=8.9 \mathrm{~Hz}, 2 \mathrm{H}), 7.75(\mathrm{~d}, J=8.7 \mathrm{~Hz}, 2 \mathrm{H}), 7.46(\mathrm{dd}, J=8.6,1.9 \mathrm{~Hz}, 2 \mathrm{H})$, $7.37(\mathrm{~d}, J=8.9 \mathrm{~Hz}, 2 \mathrm{H}), 7.23(\mathrm{~d}, J=2.0 \mathrm{~Hz}, 2 \mathrm{H}), 5.10(\mathrm{~s}, 2 \mathrm{H})$.

(rac)-[9,9'-Biphenanthrene]-10,10'-diol (10)

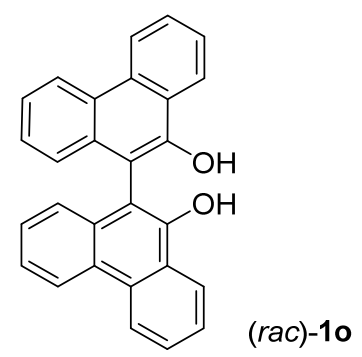

Compound ( $r a c)-10$ was obtained following a reported method described in the literature. ${ }^{11}$

${ }^{1} \mathrm{H} \mathrm{NMR}\left(300 \mathrm{MHz}, \mathrm{CDCl}_{3}\right) \delta: 8.87(\mathrm{~d}, J=8.4 \mathrm{~Hz}, 2 \mathrm{H}), 8.81(\mathrm{~d}, J=8.3 \mathrm{~Hz}, 2 \mathrm{H}), 8.54(\mathrm{~d}, J=8.1 \mathrm{~Hz}, 2 \mathrm{H}), 7.88(\mathrm{t}$, $J=7.6 \mathrm{~Hz}, 2 \mathrm{H}), 7.78(\mathrm{t}, J=7.5 \mathrm{~Hz}, 2 \mathrm{H}), 7.59(\mathrm{t}, J=7.5 \mathrm{~Hz}, 2 \mathrm{H}), 7.41(\mathrm{t}, J=7.5 \mathrm{~Hz}, 2 \mathrm{H}), 7.37-7.22(\mathrm{~m}, 2 \mathrm{H})$, $5.67(\mathrm{~s}, 2 \mathrm{H})$.

${ }^{11}$ Z. He, A. P. Pulis, D. J. Procter, Angew. Chem., Int. Ed. 2019, 58, 7813. 


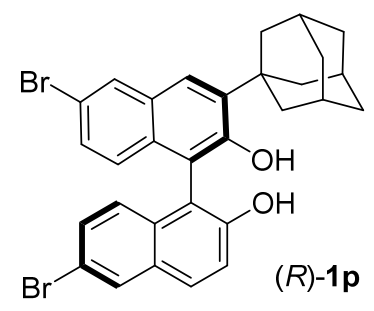

Concentrated $\mathrm{H}_{2} \mathrm{SO}_{4}(0.12 \mathrm{~mL}, 2.08 \mathrm{mmol})$ was added dropwise to a stirred solution of binol $(R)-\mathbf{1 b}$ (ee $>99 \%$ ) (150 mg, $0.34 \mathrm{mmol}$ ) and 1-adamantanol (158.3 mg, $1.04 \mathrm{mmol})$ in $\mathrm{CH}_{2} \mathrm{Cl}_{2}(2.6 \mathrm{~mL})$ at 0 을 min. After stirring for $30 \mathrm{~min}$ at $0 \stackrel{\circ}{ }{ }^{\circ}$, the ice bath was removed. The reaction mixture was stirred at $\mathrm{rt}$ for $47 \mathrm{~h}$ and quenched with aqueous $\mathrm{NaOH}(5 \%)$. The resulting mixture was extracted with $\mathrm{CH}_{2} \mathrm{Cl}_{2}(\times 3)$ and the combined organic layers were washed with brine and dried with $\mathrm{Na}_{2} \mathrm{SO}_{4}$ and concentrated. The crude solid was purified by silica gel chromatography using heptane/AcOEt (3:1) to give binol (R)-1p in $46 \%$ yield $(91.0 \mathrm{mg}, 0.16 \mathrm{mmol})$, as a yellow solid.

${ }^{1} \mathrm{H}$ NMR $\left(300 \mathrm{MHz}, \mathrm{CDCl}_{3}\right) \delta: 8.05(\mathrm{dd}, J=4.8,1.7 \mathrm{~Hz}, 2 \mathrm{H}), 7.90(\mathrm{~d}, J=9.0 \mathrm{~Hz}, 1 \mathrm{H}), 7.79(\mathrm{~s}, 1 \mathrm{H}), 7.41(\mathrm{~d}, J=$ 8.5, $1 \mathrm{H}), 7.39$ (dd, $J=8.6,1.7 \mathrm{~Hz}, 1 \mathrm{H}), 7.31(\mathrm{dd}, J=8.8,1.7 \mathrm{~Hz}, 1 \mathrm{H}), 6.96(\mathrm{~d}, J=9.1 \mathrm{~Hz}, 1 \mathrm{H}), 6.86(\mathrm{~d}, J=8.8$ $H z, 1 H), 5.29(s, 1 H), 5.08(s, 1 H), 2.26(s, 6 H), 2.14(s, 3 H), 1.82(s, 6 H)$.

${ }^{13} \mathrm{C}$ NMR (126 MHz, CDCl $)$ ) 8: 153.3, 152.9, 140.1, 132.0, 131.0, 130.8, 130.7, 130.6, 130.5, 130.4, 130.1, $130.0,127.2,125.8,125.2,119.0,118.1,117.6,111.0,110.8,40.5$ (3C), 37.8, 37.0 (3C), 29.0 (3C).

HRMS (ESI): calculated for $\mathrm{C}_{30} \mathrm{H}_{26} \mathrm{Br}_{2} \mathrm{NaO}_{2}\left([\mathrm{M}+\mathrm{Na}]^{+}\right)$599.0300, found 599.0317 .

$[\alpha]_{D}{ }^{20}=-27.8\left(c=0.018, \mathrm{CHCl}_{3}\right)$.

Chiral HPLC: Daicel Chiralpak IB, n-hexane / 2-propanol: 97/3, flow rate: $0.3 \mathrm{~mL} / \mathrm{min}, \lambda: 254 \mathrm{~nm}, \mathrm{t}_{\mathrm{R}}$ (major): 39.25 $\min , \mathrm{T}: 25 \stackrel{\circ}{ } \mathrm{C},>99 \%$ ee.
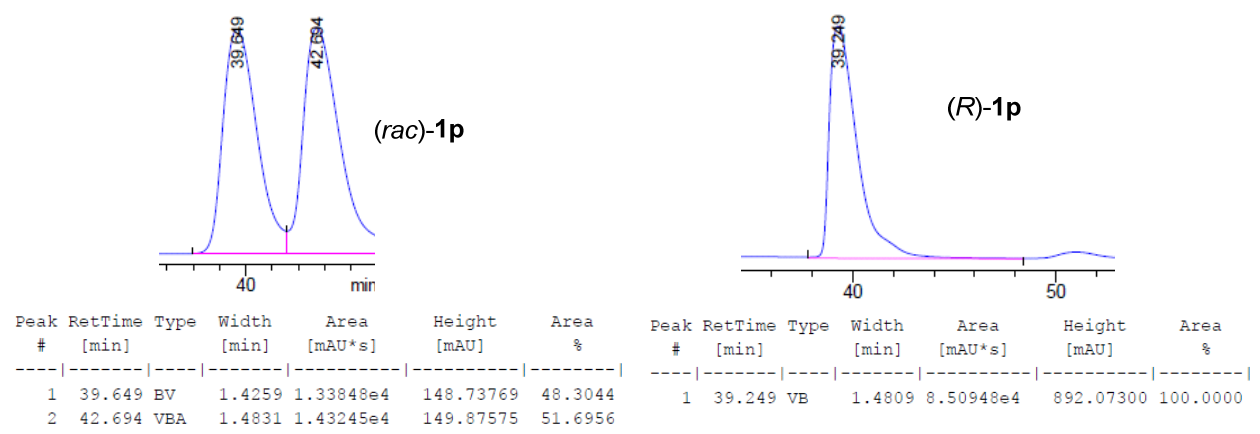
(rac)-6,6'-Dibromo-3,3'-dinitro-[1,1'-binaphthalene]-2,2'-diol (1q)<smiles>O=[N+]([O-])c1cc2cc(Br)ccc2c(-c2c(O)c([N+](=O)[O-])cc3cc(Br)ccc23)c1O</smiles>

Compound (rac)-1q was obtained following a reported method described in the literature. ${ }^{10}$ terc-Butyl nitrite $(232 \mu \mathrm{l}, 2.1 \mathrm{mmol})$ was added to a solution of binol $(\mathrm{rac})-1 \mathrm{~b}(143,2 \mathrm{mg}, 0.5 \mathrm{mmol})$ in THF $(2.5 \mathrm{~mL})$. The reaction mixture was stirred, open to the air, at $\mathrm{rt}$ for $22 \mathrm{~h}$ and the solvent evaporated. The residue was purified by flash chromatography (heptane/ $\mathrm{CH}_{2} \mathrm{Cl}_{2} 2: 1$ ) to give binol (rac)-1q in $35 \%$ yield ( $55.9 \mathrm{mg}, 0.11 \mathrm{mmol}$ ), as a brown solid.

${ }^{1} \mathrm{H}$ NMR (300 MHz, CDCl$)$ ) 8: 10.27 (s, 2H), 8.91 (bs, $\left.2 \mathrm{H}\right), 8.21$ (d, $\left.J=2.0 \mathrm{~Hz}, 2 \mathrm{H}\right), 7.53$ (dd, $J=9.1,2.0 \mathrm{~Hz}$, $2 \mathrm{H}), 7.01(\mathrm{~d}, J=9.1 \mathrm{~Hz}, 2 \mathrm{H})$.

\section{Oxidative dearomatization of binols with Oxone / $\mathrm{NaHCO}_{3} /$ acetone}

\section{Method A: General procedure for the oxidative dearomatization at room temperature.}

$\mathrm{NaHCO}_{3}$ and the corresponding binol were solved in acetone (0.1 M) and Milli-Q water (0.1 M). A solution of Oxone in Milli-Q water was added dropwise to the mixture at $\mathrm{rt}$ for $1 \mathrm{~h}$, using a syringe pump (Figure 1), and stirred at $\mathrm{rt}$ for an additional hour. The mixture was extracted with $\mathrm{CH}_{2} \mathrm{Cl}_{2}(\mathrm{x} 3)$, dried over $\mathrm{MgSO}_{4}$ and the solvent evaporated. The residue was purified by flash chromatography with neutral silica ge ${ }^{12}$ to give the desired product.

Method B: General procedure for the oxidative dearomatization at 0 ㅇ.

$\mathrm{NaHCO}_{3}$ and the corresponding binol were solved in acetone $(0.1 \mathrm{M})$ and Milli-Q water $(0.1 \mathrm{M})$. A solution of Oxone in Milli- $\mathrm{Q}$ water was added dropwise to the mixture at $0{ }^{\circ} \mathrm{C}$ for $1 \mathrm{~h}$, using a syringe pump (Figure 1 ), and stirred at rt for an additional hour. The mixture was extracted with $\mathrm{CH}_{2} \mathrm{Cl}_{2}(\mathrm{x} 3)$, dried over $\mathrm{MgSO}_{4}$ and the solvent evaporated. The residue was purified by flash chromatography with neutral silica gel to give the desired product.

${ }^{12}$ A. V. Kumar, M. Patil, A. R. Kapdi, RSC Adv. 2015, 5, 54505 (Supporting Information). 


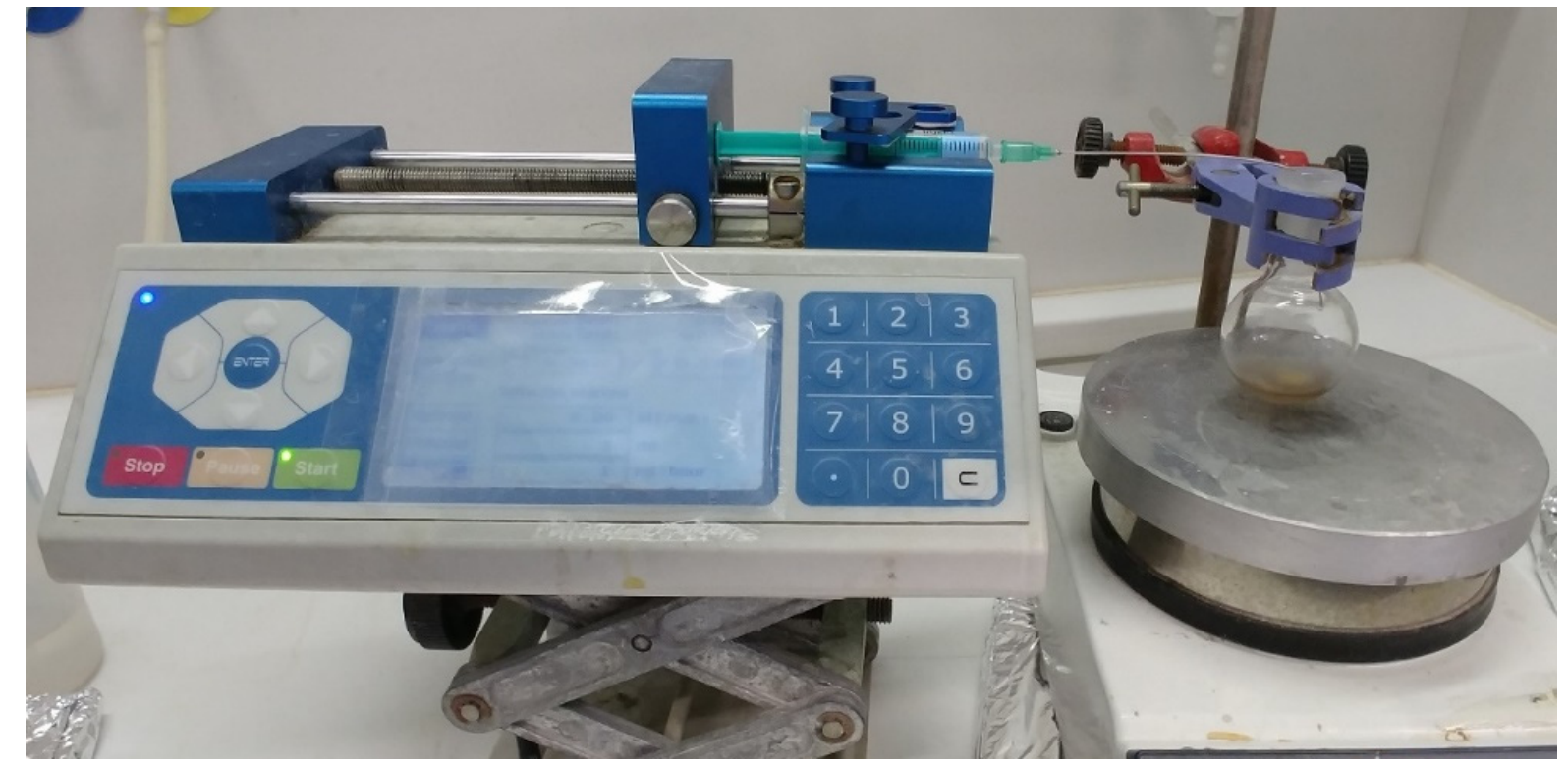

Figure 1. Addition of an aqueous solution of Oxone to the reaction mixture, using a syringe pump.

$\left(6 a R^{*}, 13 c S^{*}\right)-6 a, 13 c-D i h y d r o d i n a p h t h o\left[2,1-b: 1^{\prime}, 2 '-d\right]$ furan-6a,13c-diol (3a)

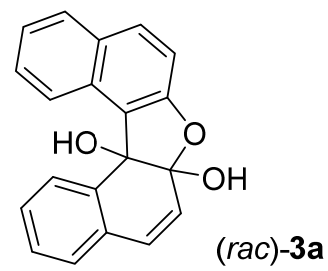

Following Method A, cis-diol ( $\mathrm{rac}$ )-3a was obtained in $66 \%$ yield (14.0 $\mathrm{mg}, 0.05 \mathrm{mmol}$ ), as a pale brown solid, from commercially available binol ( $r a c)-1 a(20.1 \mathrm{mg}, 0.07 \mathrm{mmol}), \mathrm{NaHCO}_{3}(58.8 \mathrm{mg}, 0.70 \mathrm{mmol}, 10$ equiv) and a solution of Oxone (85.9 mg, $0.28 \mathrm{mmol}, 4$ equiv) in Milli-Q water $(0.7 \mathrm{~mL})$. The crude mixture was purified by flash chromatography with neutral silica gel (heptane/EtOAc : 5/1).

${ }^{1} \mathrm{H}$ NMR $\left.\left(500 \mathrm{MHz}^{\mathrm{CDCl}}\right)_{3}\right) \delta: 8.23(\mathrm{~d}, J=7.7 \mathrm{~Hz}, 1 \mathrm{H}), 8.06(\mathrm{~d}, J=8.5 \mathrm{~Hz}, 1 \mathrm{H}), 7.78(\mathrm{t}, J=8.8 \mathrm{~Hz}, 2 \mathrm{H}), 7.42$ (td, $J=7.6,1.3 \mathrm{~Hz}, 1 \mathrm{H}), 7.39-7.32(\mathrm{~m}, 2 \mathrm{H}), 7.29(\mathrm{ddd}, J=8.0,6.8,1.0 \mathrm{~Hz}, 1 \mathrm{H}), 7.15-7.11(\mathrm{~m}, 2 \mathrm{H}), 6.60$ $(\mathrm{d}, J=9.8 \mathrm{~Hz}, 1 \mathrm{H}), 6.04(\mathrm{~d}, J=9.9 \mathrm{~Hz}, 1 \mathrm{H}), 4.90(\mathrm{~s}, 1 \mathrm{H}), 2.65(\mathrm{~s}, 1 \mathrm{H})$.

${ }^{13}$ C NMR (75 MHz, CDCl $)$ ) 8: 157.5, 134.8, 132.8, 131.4, 131.1, 130.96, 130.4, 129.4, 129.2, 128.7, 128.5, $127.9,127.5,125.1,123.5,121.6,120.5,113.0,107.7,81.2$. 
HRMS (ESI): calculated for $\mathrm{C}_{20} \mathrm{H}_{14} \mathrm{O}_{3} \mathrm{Na}\left([\mathrm{M}+\mathrm{Na}]^{+}\right) 325.0835$, found 325.0829 .

HPLC: Daicel Chiralpak IC, $n$-hexane/2-propanol: 90/10, flow rate: $1.0 \mathrm{~mL} / \mathrm{min}, \lambda: 254 \mathrm{~nm}, t_{R}: 17.64 \mathrm{~min}$, $t_{R}: 21.34 \min ., \mathrm{T}: 25^{\circ} \mathrm{C}$.

(6aR,13cS)-6a,13c-Dihydrodinaphtho[2,1-b:1',2'-d]furan-6a,13c-diol (3a)

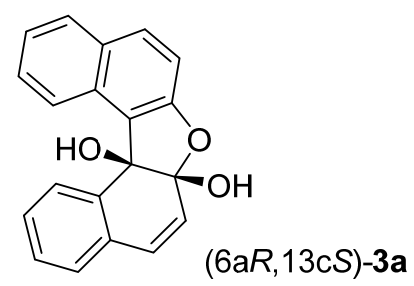

Following Method A, cis-diol (6aR,13cS)-3a was obtained in $66 \%$ yield (13.1 mg, $0.05 \mathrm{mmol}$ ), as a pale brown solid, from commercially available binol $(R)-1 \mathrm{a}(20.1 \mathrm{mg}, 0.07 \mathrm{mmol}), \mathrm{NaHCO}_{3}(58.8 \mathrm{mg}, 0.70 \mathrm{mmol}$, 10 equiv) and a solution of Oxone ( $85.9 \mathrm{mg}, 0.28 \mathrm{mmol}, 4$ equiv) in Milli-Q water ( $0.7 \mathrm{~mL})$. The crude mixture was purified by flash chromatography with neutral silica gel (heptane/EtOAc : 5/1).

$[\alpha]_{\mathrm{D}}{ }^{20}=-126.0\left(c=0.04, \mathrm{CHCl}_{3}\right)$.

HPLC: Daicel Chiralpak IC, $n$-hexane/2-propanol: 90/10, flow rate: $1.0 \mathrm{~mL} / \mathrm{min}, \lambda: 254 \mathrm{~nm}, t_{R}$ (major): 17.52 $\min , t_{R}$ (minor): $21.27 \min ., \mathrm{T}: 25^{\circ} \mathrm{C}, 93 \%$ ee.
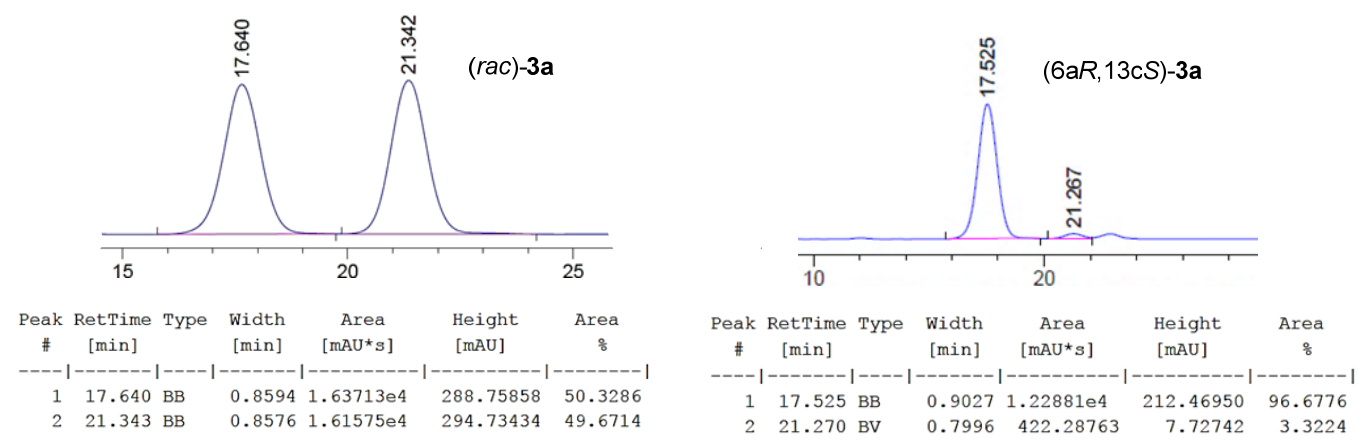

Following Method B, cis-diol (6aR,13cS)-3a was obtained in $77 \%$ yield (16.6 mg, $0.06 \mathrm{mmol}$ ), as a pale brown solid, from commercially available binol (R)-1a (ee >99\%) (20.5 mg, $0.07 \mathrm{mmol}$ ), $\mathrm{NaHCO}_{3}(58.8 \mathrm{mg}$, $0.70 \mathrm{mmol}, 10$ equiv) and a solution of Oxone $(85.9 \mathrm{mg}, 0.28 \mathrm{mmol}, 4$ equiv) in Milli-Q water ( $0.7 \mathrm{~mL})$. The crude mixture was purified by flash chromatography with neutral silica gel (heptane/EtOAc : 5/1). 
$[\alpha]_{\mathrm{D}}{ }^{20}=-136.2\left(c=0.037, \mathrm{CHCl}_{3}\right)$.

HPLC: Daicel Chiralpak IC, $n$-hexane/2-propanol: 90/10, flow rate: $1.0 \mathrm{~mL} / \mathrm{min}, \lambda: 254 \mathrm{~nm}, t_{R}$ (major): 18.06 $\min , t_{R}$ (minor): $21.84 \min ., \mathrm{T}: 25^{\circ} \mathrm{C}, 98 \%$ ee.
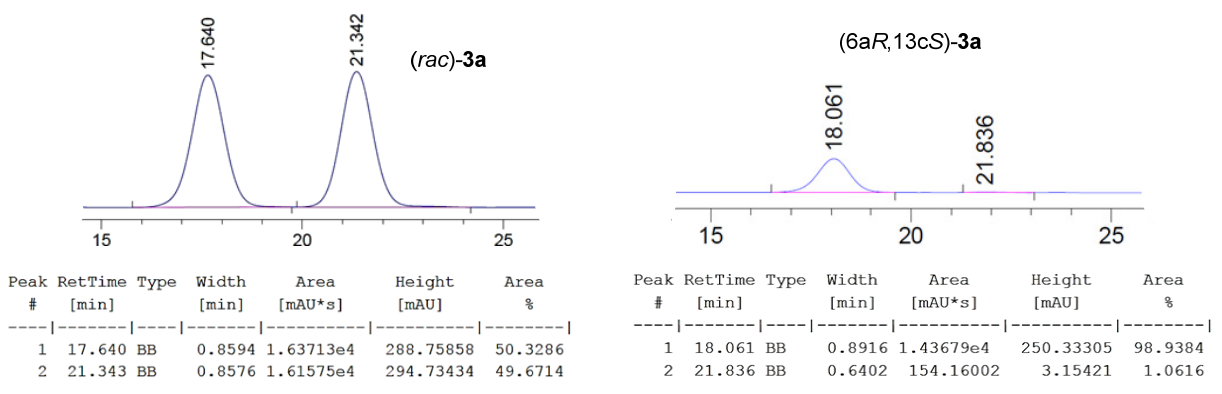

(6aS,13cR)-6a,13c-Dihydrodinaphtho[2,1-b:1',2'-d]furan-6a,13c-diol (3a)

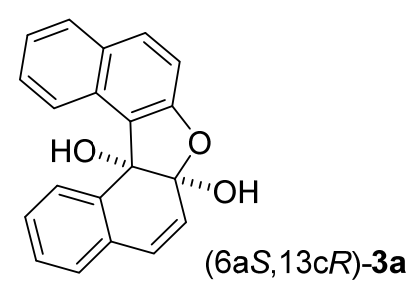

Following Method B, cis-diol (6aS,13cR)-3a was obtained in $69 \%$ yield (12.3 mg, $0.04 \mathrm{mmol})$, as a brown solid, from commercially available binol (S)-1a (ee >99\%) (19.8 mg, $0.07 \mathrm{mmol}$ ), $\mathrm{NaHCO}_{3}(58.8 \mathrm{mg}, 0.70$ mmol, 10 equiv) and a solution of Oxone ( $85.9 \mathrm{mg}, 0.28 \mathrm{mmol}, 4$ equiv) in Milli-Q water $(0.7 \mathrm{~mL})$. The crude mixture was purified by flash chromatography with neutral silica gel (heptane/EtOAc : 5/1).

$[\alpha]_{\mathrm{D}}{ }^{20}=+119.3\left(c=0.008, \mathrm{CHCl}_{3}\right)$.

HPLC: Daicel Chiralpak IC, $n$-hexane/2-propanol: 90/10, flow rate: $1.0 \mathrm{~mL} / \mathrm{min}, \lambda: 254 \mathrm{~nm}, t_{R}$ (minor): 17.95 $\min , t_{R}$ (major): $21.62 \min ., \mathrm{T}: 25^{\circ} \mathrm{C}, \mathbf{9 6 \%}$ ee.
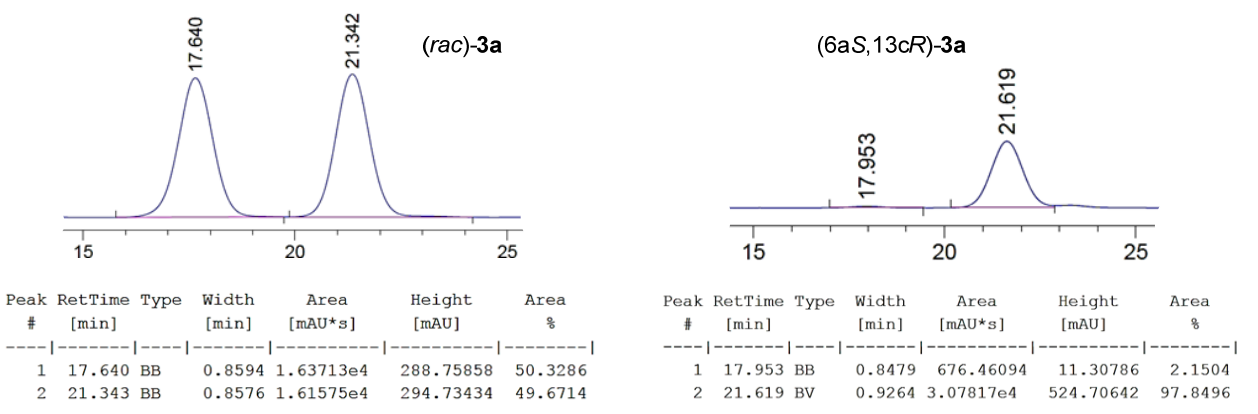
$\left(6 a R^{*}, 13 c S^{*}\right)-3,11-D i b r o m o-6 a, 13 c-d i h y d r o d i n a p h t h o\left[2,1-b: 1^{\prime}, 2 '-d\right]$ furan-6a,13c-diol (3b)<smiles>OC12C=Cc3cc(Br)ccc3C1(O)c1c(ccc3cc(Br)ccc13)O2</smiles>

Following Method A, cis-diol ( $\mathrm{rac}$ )-3b was obtained in $51 \%$ yield ( $21.5 \mathrm{mg}, 0.05 \mathrm{mmol})$, as a white solid, from binol (rac)-1b (40.8 mg, $0.09 \mathrm{mmol}), \mathrm{NaHCO}_{3}(75.6 \mathrm{mg}, 0.90 \mathrm{mmol}, 10$ equiv) and a solution of Oxone (110.7 mg, 0.36 $\mathrm{mmol}, 4$ equiv) in Milli-Q water $(0.90 \mathrm{~mL})$. The crude mixture was purified by flash chromatography with neutral silica gel (heptane/EtOAc 5:1).

${ }^{1} \mathrm{H} \mathrm{NMR}\left(300 \mathrm{MHz}, \mathrm{CDCl}_{3}\right) \delta: 8.03(\mathrm{~d}, J=8.3 \mathrm{~Hz}, 1 \mathrm{H}), 7.93(\mathrm{~d}, J=2.1 \mathrm{~Hz}, 1 \mathrm{H}), 7.85(\mathrm{~d}, J=9.0 \mathrm{~Hz}, 1 \mathrm{H}), 7.70$ $(\mathrm{d}, J=8.8 \mathrm{~Hz}, 1 \mathrm{H}), 7.54(\mathrm{dd}, J=8.3,2.1 \mathrm{~Hz}, 1 \mathrm{H}), 7.46$ (dd, $J=9.1,2.1 \mathrm{~Hz}, 1 \mathrm{H}), 7.27$ (bs, $1 \mathrm{H}), 7.13$ (d, $J=8.7$ $\mathrm{Hz}, 1 \mathrm{H}), 6.53(\mathrm{~d}, J=9.9 \mathrm{~Hz}, 1 \mathrm{H}), 6.07(\mathrm{~d}, J=9.9 \mathrm{~Hz}, 1 \mathrm{H}), 4.82(\mathrm{~s}, 1 \mathrm{H}), 2.68(\mathrm{~s}, 1 \mathrm{H})$.

${ }^{13} \mathrm{C} \mathrm{NMR}\left(75 \mathrm{MHz}, \mathrm{CDCl}_{3}\right.$ ) $\delta: 157.2,133.5,133.2,131.8,131.5,131.3,131.2,130.8,130.58,130.2,129.9$, $129.2,126.3,123.3,123.1,120.3,117.3,113.9,107.5,80.6$.

HRMS (ESI): calculated for $\mathrm{C}_{20} \mathrm{H}_{12} \mathrm{O}_{3} \mathrm{Br}_{2} \mathrm{Na}\left([\mathrm{M}+\mathrm{Na}]^{+}\right) 480.9045$, found 480.9055 .

Chiral HPLC: Daicel Chiralpak IC, $n$-hexane / 2-propanol: 90/10, flow rate: $0.5 \mathrm{~mL} / \mathrm{min}, \lambda: 254 \mathrm{~nm}, t_{R}: 19.58 \mathrm{~min}$, $t_{R}: 22.14 \min ., \mathrm{T}: 25^{\circ} \mathrm{C}$.

(6aR,13cS)-3,11-Dibromo-6a,13c-dihydrodinaphtho[2,1-b:1',2'-d]furan-6a,13c-diol (3b)<smiles></smiles> 
To a solution of (R)-6,6'-dibromo-[1,1'-binaphthalene]-2,2'-diol (1b) (1.0 g, $2.26 \mathrm{mmol})$ in acetone (0.1 M, 22.6 $\mathrm{mL}$ ), a solution of $\mathrm{NaHCO}_{3}(2.85 \mathrm{~g}, 33.94 \mathrm{mmol}, 15$ equiv) in Milli-Q water (0.1 M, $22.6 \mathrm{ml})$ was added. To this mixture, a solution of Oxone $(4.17 \mathrm{~g}, 13.58 \mathrm{mmol}, 6$ equiv) in Milli-Q water $(0.1 \mathrm{M}, 22.6 \mathrm{~mL})$ was added dropwise at $0 \stackrel{\circ}{ } \mathrm{C}$ for $1 \mathrm{~h}$, using a syringe pump, and stirred at rt for an additional hour. After that, the crude mixture was extracted with $\mathrm{CH}_{2} \mathrm{Cl}_{2}(\mathrm{x} 3)$, dried over $\mathrm{MgSO}_{4}$ and concentrated to dryness, under reduced pressure. The residue was purified by flash chromatography with neutral silica gel $\left(\mathrm{CH}_{2} \mathrm{Cl}_{2} / \mathrm{EtOAc} 2: 1\right)$ to give cis-diol $(6 \mathrm{a} R, 13 \mathrm{cS})-3 \mathbf{b}$ in $46 \%$ yield ( $480.60 \mathrm{mg}, 1.05 \mathrm{mmol}$ ), as a white solid.

$[\alpha]_{D}{ }^{20}=+21.4\left(c=0.013, \mathrm{CHCl}_{3}\right)$.

Chiral HPLC: Daicel Chiralpak IC, $n$-hexane / 2-propanol: 90/10, flow rate: $0.5 \mathrm{~mL} / \mathrm{min}, \lambda: 254 \mathrm{~nm}, t_{R}$ (major): 17.37 $\min , t_{R}$ (minor): $19.59 \min ., \mathrm{T}: 25^{\circ} \mathrm{C}, 97 \%$ ee.
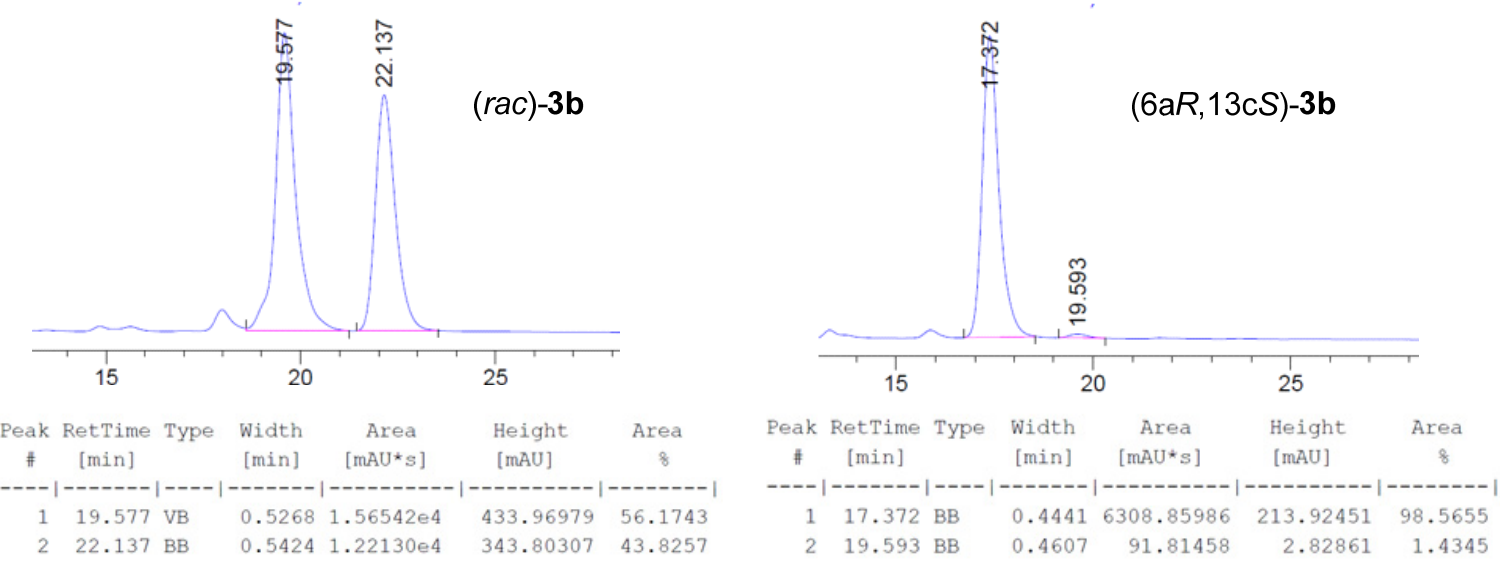

$\left(6 a R^{*}, 13 c S^{*}\right)-3,11-D i m e t h y l d i n a p h t h o\left[2,1-b: 1^{\prime}, 2^{\prime}-d\right]$ furan-6a,13c-diol (3c)

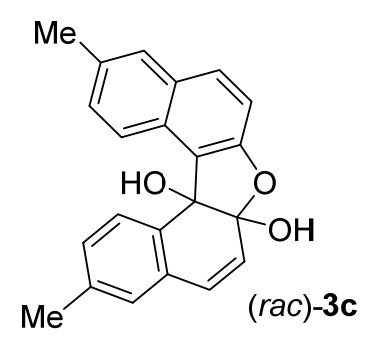


Following Method A, (rac)-3c was obtained in $64 \%$ yield (13.5 mg, $0.04 \mathrm{mmol})$, as a yellow solid, from binol (rac)1c (20.0 mg, $0.06 \mathrm{mmol}), \mathrm{NaHCO}_{3}(53.5 \mathrm{mg}, 0.64 \mathrm{mmol}, 10$ equiv) and a solution of Oxone (78.0 mg, $0.25 \mathrm{mmol}$, 4 equiv) in Milli-Q water ( $0.64 \mathrm{~mL})$. The crude mixture was purified by flash chromatography with neutral silica gel $\left(\mathrm{CH}_{2} \mathrm{Cl}_{2} / \mathrm{EtOAc} 13: 1\right)$.

${ }^{1} \mathrm{H}$ NMR $\left(300 \mathrm{MHz}, \mathrm{CDCl}_{3}\right) \delta: 8.09(\mathrm{~d}, J=7.9 \mathrm{~Hz}, 1 \mathrm{H}), 8.01(\mathrm{~d}, J=8.6 \mathrm{~Hz}, 1 \mathrm{H}), 7.70(\mathrm{~d}, J=8.7 \mathrm{~Hz}, 1 \mathrm{H}), 7.55$ (bs, 1H), $7.24(\mathrm{~m}, 2 \mathrm{H}), 7.10(\mathrm{~d}, J=8.8 \mathrm{~Hz}, 1 \mathrm{H}), 6.94(\mathrm{bs}, 1 \mathrm{H}), 6.56(\mathrm{~d}, J=9.8 \mathrm{~Hz}, 1 \mathrm{H}), 6.04(\mathrm{~d}, J=9.8 \mathrm{~Hz}, 1 \mathrm{H})$, $4.90(\mathrm{~s}, 1 \mathrm{H}), 2.59(\mathrm{~s}, 1 \mathrm{H}), 2.44(\mathrm{~s}, 3 \mathrm{H}), 2.36(\mathrm{~s}, 3 \mathrm{H})$.

${ }^{13} \mathrm{C}$ NMR (126 MHz, $\left.\mathrm{CDCl}_{3}\right)$ ): 156.6, 138.8, 132.8, 131.9, 131.8, 131.1, 131.0, 130.6, 129.5, 129.0, 128.9, $128.5,128.5,128.2,125.1,121.4,120.4,112.8,107.5,81.0,21.3,21.1$.

HRMS (ESI): calculated for $\mathrm{C}_{22} \mathrm{H}_{20} \mathrm{NaO}_{3}\left([\mathrm{M}+\mathrm{Na}]^{+}\right) 355.1148$, found 353.1151 .

Chiral HPLC: Daicel Chiralpak IC, $n$-hexane / 2-propanol: 90/10, flow rate: $1.0 \mathrm{~mL} / \mathrm{min}, \lambda: 254 \mathrm{~nm}, t_{R}: 23.32 \mathrm{~min}$, $t_{R}: 29.41 \mathrm{~min} ., \mathrm{T}: 25^{\circ} \mathrm{C}$.

(6aR,13cS)-3,11-Dimethyldinaphtho[2,1-b:1',2'-d]furan-6a,13c-diol (3c)

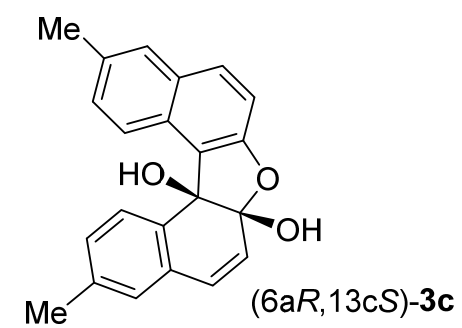

Following Method B, $(6 a R, 13 \mathrm{cS})-3 \mathrm{c}$ was obtained in $56 \%$ yield $(24.0 \mathrm{mg}, 0.07 \mathrm{mmol})$, as a yellow solid, from binol (R)-1c (ee 99\%) (41.3 mg, $0.13 \mathrm{mmol}), \mathrm{NaHCO}_{3}(165.5 \mathrm{mg}, 1.97 \mathrm{mmol}, 15$ equiv) and a solution of Oxone (242.3 $\mathrm{mg}, 0.79 \mathrm{mmol}, 6$ equiv) in Milli-Q water $(1.3 \mathrm{~mL})$. The crude mixture was purified by flash chromatography with neutral silica gel $\left(\mathrm{CH}_{2} \mathrm{Cl}_{2} / \mathrm{EtOAc} 1: 1\right)$.

$[\alpha]_{\mathrm{D}}{ }^{20}=+17.4\left(\mathrm{c} 0.023, \mathrm{CHCl}_{3}\right)$.

Chiral HPLC: Daicel Chiralpak IC, $n$-hexane / 2-propanol: 90/10, flow rate: $1.0 \mathrm{~mL} / \mathrm{min}, \lambda: 254 \mathrm{~nm}, t_{R}: 23.32 \mathrm{~min}$, $\mathrm{T}: 25^{\circ} \mathrm{C},>99 \%$ ee. 

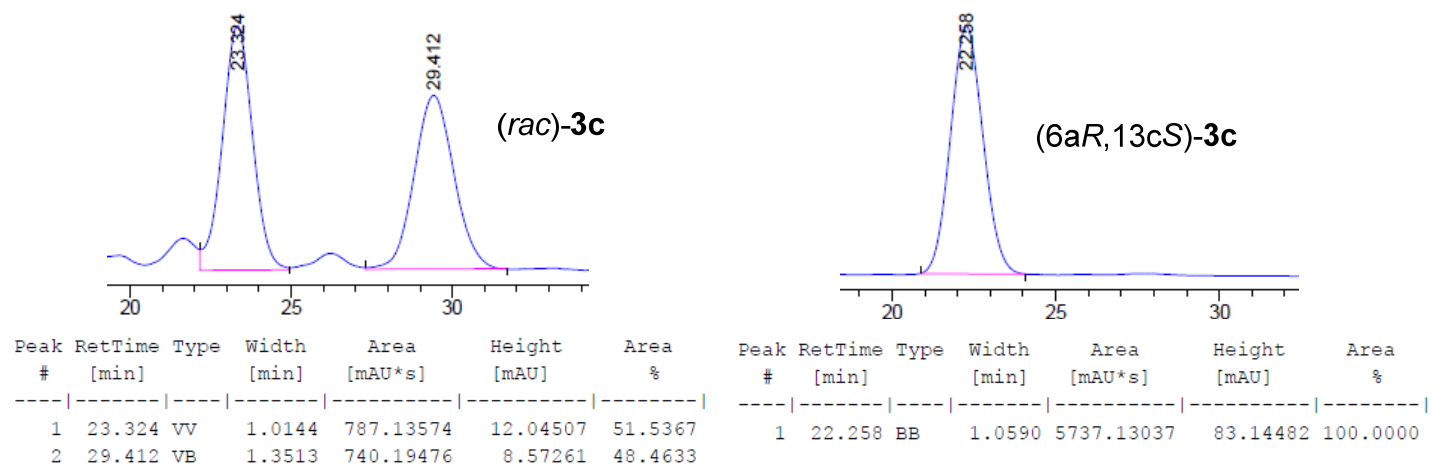

$\left(6 a R^{*}, 13 c S^{*}\right)-3,11-D i e t h y l d i n a p h t h o\left[2,1-b: 1^{\prime}, 2^{\prime}-d\right] f u r a n-6 a, 13 c-d i o l(3 d)$

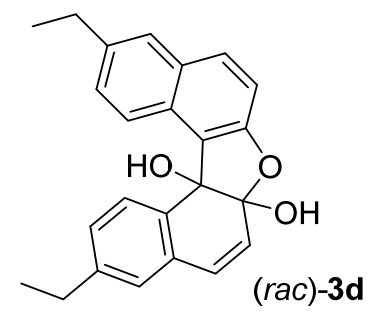

Following Method A, ( $\mathrm{rac}$ )-3d was obtained in $62 \%$ yield ( $17.4 \mathrm{mg}, 0.05 \mathrm{mmol}$ ), as a yellow solid, from binol ( $\mathrm{rac}$ )$1 \mathrm{~d}(27.0 \mathrm{mg}, 0.08 \mathrm{mmol}), \mathrm{NaHCO}_{3}(66.2 \mathrm{mg}, 0.79 \mathrm{mmol}, 10$ equiv) and a solution of Oxone ( $96.9 \mathrm{mg}, 0.31 \mathrm{mmol}$, 4 equiv) in Milli-Q water $(0.8 \mathrm{~mL})$. The crude mixture was purified by preparative TLC with neutral silica gel (heptane/EtOAc 1:2).

${ }^{1} \mathrm{H}$ NMR $\left(300 \mathrm{MHz}, \mathrm{CDCl}_{3}\right) \delta: 8.12(\mathrm{~d}, J=8.0 \mathrm{~Hz}, 1 \mathrm{H}), 8.05(\mathrm{~d}, J=8.7 \mathrm{~Hz}, 1 \mathrm{H}), 7.72(\mathrm{~d}, J=8.8 \mathrm{~Hz}, 1 \mathrm{H}), 7.56$ (bs, 1H), $7.30-7.21(\mathrm{~m}, 2 \mathrm{H}), 7.11(\mathrm{~d}, J=8.8 \mathrm{~Hz}, 1 \mathrm{H}), 6.96(\mathrm{~s}, 1 \mathrm{H}), 6.58(\mathrm{~d}, J=9.8 \mathrm{~Hz}, 1 \mathrm{H}), 6.04(\mathrm{~d}, J=9.8$ $\mathrm{Hz}, 1 \mathrm{H}), 4.90(\mathrm{~s}, 1 \mathrm{H}), 2.73(\mathrm{q}, J=7.4 \mathrm{~Hz}, 2 \mathrm{H}), 2.65(\mathrm{q}, J=7.9 \mathrm{~Hz}, 2 \mathrm{H}), 2.59(\mathrm{~s}, 1 \mathrm{H}), 1.31-1.18(\mathrm{~m}, 6 \mathrm{H})$.

${ }^{13} \mathrm{C}$ NMR (126 MHz, CDCl $)$ ) 8: 156.8, 145.3, 139.3, 132.1, 132.1, 131.3, 131.2, 130.8, 129.3, 128.8, 128.6, $128.0,127.5,127.1,125.1,121.7,120.5,112.9,107.6,81.2,28.8,28.6,15.7,15.5$.

HRMS (ESI): calculated for $\mathrm{C}_{24} \mathrm{H}_{22} \mathrm{NaO}_{3}\left([\mathrm{M}+\mathrm{Na}]^{+}\right) 381.1461$, found 381.1458 .

Chiral HPLC: Daicel Chiralpak IC, n-hexane / 2-propanol: 92/8, flow rate: $1.0 \mathrm{~mL} / \mathrm{min}, \lambda: 230 \mathrm{~nm}, \mathrm{t}_{\mathrm{R}}: 24.62$ $\min , \mathrm{t}_{\mathrm{R}}: 29.04 \mathrm{~min}, \mathrm{~T}: 25^{\circ} \mathrm{C}$. 
(6aR,13cS)-3,11-Diethyldinaphtho[2,1-b:1',2'-d]furan-6a,13c-diol (3d)

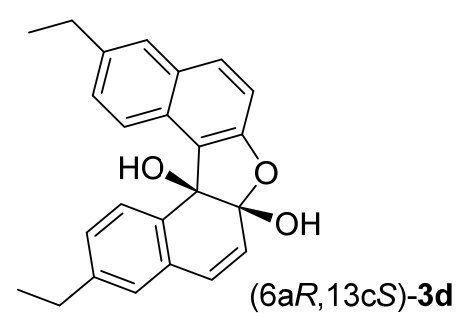

Following Method A, (6aR,13cS)-3d was obtained in $57 \%$ yield $(23.1 \mathrm{mg}, 0.06 \mathrm{mmol})$, as a yellow solid, from binol (R)-1d (ee 95\%) (38.7 mg, $0.11 \mathrm{mmol}$ ), $\mathrm{NaHCO}_{3}(142.4 \mathrm{mg}, 1.69 \mathrm{mmol}, 15$ equiv) and a solution of Oxone (208.4 $\mathrm{mg}, 0.68 \mathrm{mmol}, 6$ equiv) in Milli-Q water $(1.13 \mathrm{~mL})$. The crude mixture was purified by preparative TLC with neutral silica gel (heptane/EtOAc 1:2).

$[\alpha]_{\mathrm{D}}{ }^{20}=+175.0\left(c=0.012, \mathrm{CHCl}_{3}\right)$.

Chiral HPLC: Daicel Chiralpak IC, $n$-hexane / 2-propanol: 92/8, flow rate: $1.0 \mathrm{~mL} / \mathrm{min}, \lambda: 230 \mathrm{~nm}, t_{R}$ (major): 25.11 $\min , t_{R}(\operatorname{minor}): 29.61 \mathrm{~min}, \mathrm{~T}: 25^{\circ} \mathrm{C}, 95 \%$ ee.
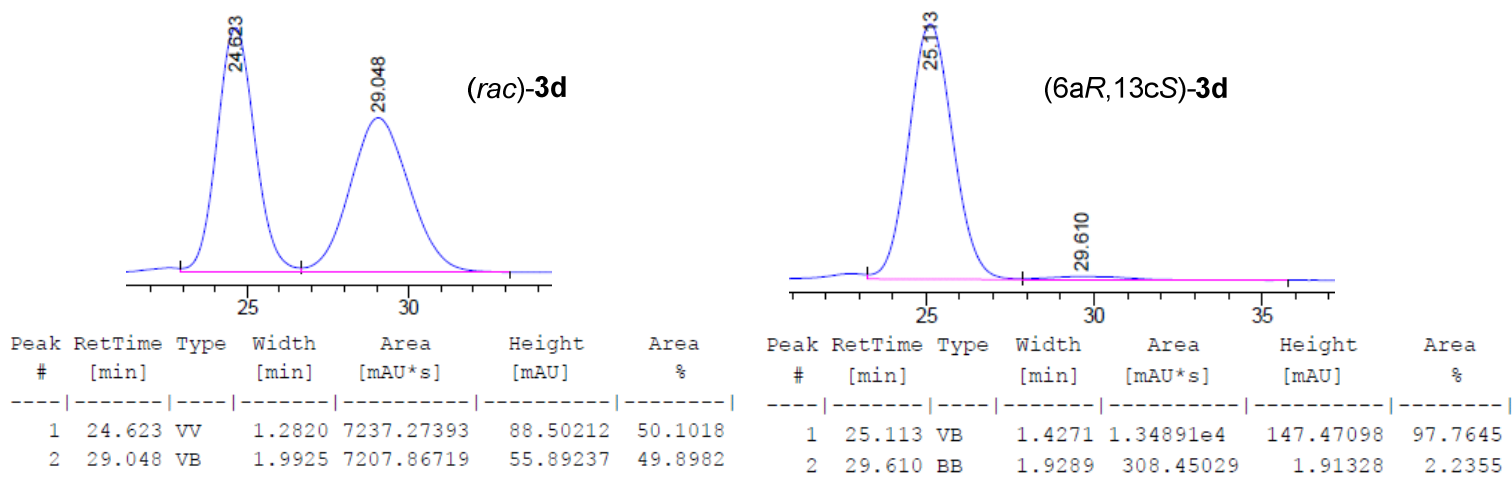

$\left(6 a R^{*}, 13 c S^{*}\right)-3,11-D i h e x y l d i n a p h t h o\left[2,1-b: 1^{\prime}, 2^{\prime}-d\right]$ furan-6a,13c-diol (3e)

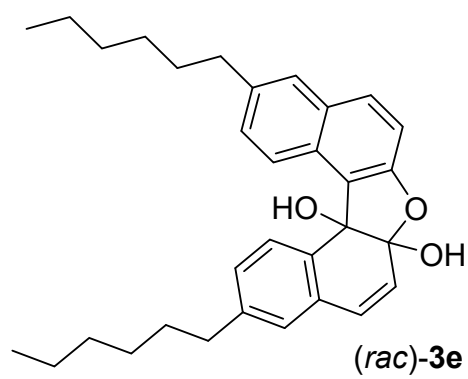


Following Method A, (rac)-3e was obtained in $55 \%$ yield ( $18.9 \mathrm{mg}, 0.04 \mathrm{mmol})$, as a yellow solid, from binol (rac)1e (33.1 mg, $0.07 \mathrm{mmol}), \mathrm{NaHCO}_{3}(61.2 \mathrm{mg}, 0.73 \mathrm{mmol}, 10$ equiv) and a solution of Oxone ( $89.5 \mathrm{mg}, 0.29 \mathrm{mmol}$, 4 equiv) in Milli-Q water $(0.7 \mathrm{~mL})$. The crude mixture was purified by preparative TLC with neutral silica gel (heptane/EtOAc 1:2).

${ }^{1} \mathrm{H}$ NMR $\left(300 \mathrm{MHz}, \mathrm{CDCl}_{3}\right) \delta: 8.10(\mathrm{~d}, J=7.9 \mathrm{~Hz}, 1 \mathrm{H}), 8.02(\mathrm{~d}, J=8.7 \mathrm{~Hz}, 1 \mathrm{H}), 7.71(\mathrm{~d}, J=8.8 \mathrm{~Hz}, 1 \mathrm{H}), 7.54$ $(\mathrm{d}, J=1.8 \mathrm{~Hz}, 1 \mathrm{H}), 7.23(\mathrm{td}, J=8.5,7.9,2.0 \mathrm{~Hz}, 2 \mathrm{H}), 7.10(\mathrm{~d}, J=8.8 \mathrm{~Hz}, 1 \mathrm{H}), 6.93(\mathrm{~d}, J=1.8 \mathrm{~Hz}, 1 \mathrm{H}), 6.57(\mathrm{~d}$, $J=9.9 \mathrm{~Hz}, 1 \mathrm{H}), 6.03(\mathrm{~d}, J=9.8 \mathrm{~Hz}, 1 \mathrm{H}), 4.90(\mathrm{~s}, 1 \mathrm{H}), 2.71-2.64(\mathrm{~m}, 2 \mathrm{H}), 2.63-2.56(\mathrm{~m}, 3 \mathrm{H}), 1.72-1.52$ $(m, 4 H), 1.34-1.26(m, 12 H), 0.94-0.83(m, 6 H)$.

${ }^{13} \mathrm{C}$ NMR (76 MHz, CDCl $)$ ) 8: 156.6, 143.9, 137.9, 131.9 (2C), 131.1 (2C), 130.6, 129.1, 128.8, 128.5, 128.4, 127.9, 127.6, 124.9, 121.5, 120.4, 112.7, 107.5, 81.1, 35.8, 35.5, 31.7, 31.6, 31.3, 31.1, 29.0, 28.9, 22.6 (2C), $14.1(2 \mathrm{C})$.

HRMS (ESI): calculated for $\mathrm{C}_{32} \mathrm{H}_{38} \mathrm{NaO}_{3}\left([\mathrm{M}+\mathrm{Na}]^{+}\right) 493.2713$, found 493.2735 .

Chiral HPLC: Daicel Chiralpak IC, n-hexane / 2-propanol: 96/4, flow rate: $1.0 \mathrm{~mL} / \mathrm{min}, \lambda: 254 \mathrm{~nm}, \mathrm{t}_{\mathrm{R}}: 44.12$ $\min , \mathrm{t}_{\mathrm{R}}: 49.46 \mathrm{~min}, \mathrm{~T}: 25^{\circ} \mathrm{C}$.

(6aR,13cS)-3,11-Dihexyldinaphtho[2,1-b:1',2'-d]furan-6a,13c-diol (3e)

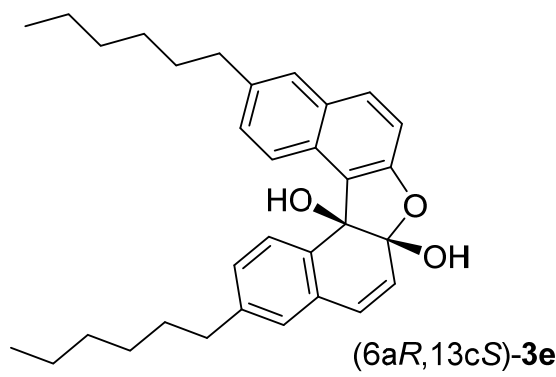

Following Method A, $(6 a R, 13 \mathrm{cS})$-3e was obtained in $60 \%$ yield $(20.0 \mathrm{mg}, 0.04 \mathrm{mmol})$, as a yellow solid, from binol (R)- 1e (ee 97\%) (32.1 mg, $0.07 \mathrm{mmol}), \mathrm{NaHCO}_{3}(89.0 \mathrm{mg}, 1.06 \mathrm{mmol}, 15$ equiv) and a solution of Oxone (130.2 $\mathrm{mg}, 0.42 \mathrm{mmol}, 6$ equiv) in Milli- $\mathrm{Q}$ water $(0.7 \mathrm{~mL})$. The crude mixture was purified by preparative TLC with neutral silica gel (heptane/EtOAc 1:2).

$[\alpha]_{D}^{20}=+65.2\left(c=0.023, \mathrm{CHCl}_{3}\right)$. 
Chiral HPLC: Daicel Chiralpak IC, $n$-hexane / 2-propanol: 96/4, flow rate: $1.0 \mathrm{~mL} / \mathrm{min}, \lambda: 254 \mathrm{~nm}, t_{R}$ (major): 43.21 $\min , t_{R}$ (minor): $48.59 \min , \mathrm{T}: 25^{\circ} \mathrm{C}, 96 \%$ ee.
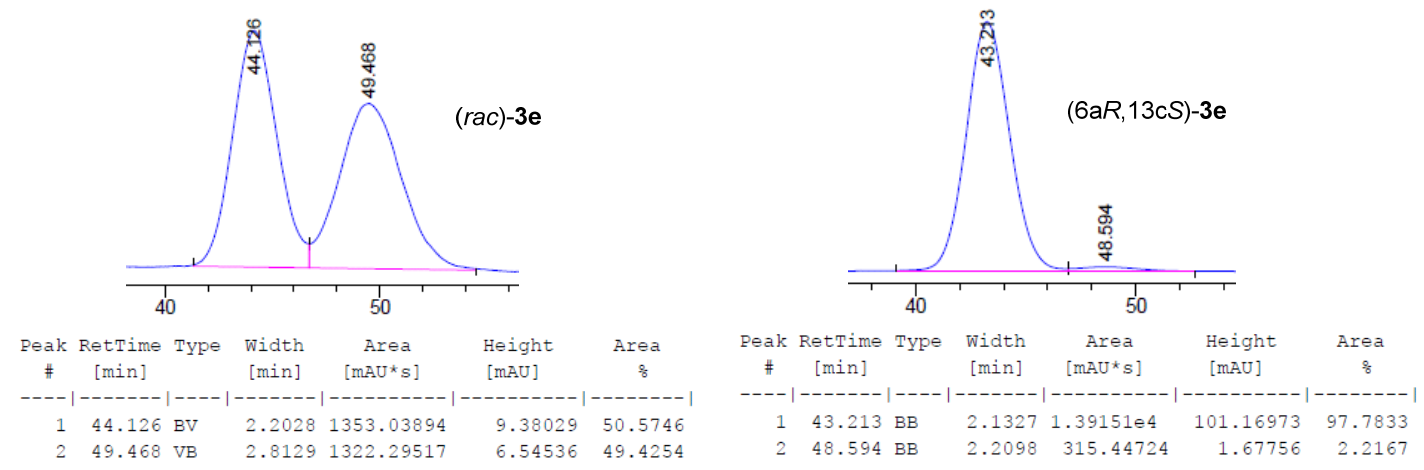

$\left(6 a R^{*}, 13 c S^{*}\right)-3,11-D i i s o p r o p y l d i n a p h t h o[2,1-b: 1 ', 2 '-d] f u r a n-6 a, 13 c-d i o l$ (3f)

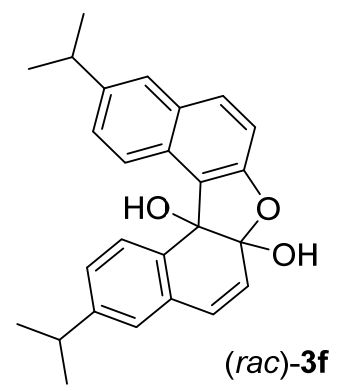

Following Method A, (rac)-3f was obtained in $58 \%$ yield ( $22.8 \mathrm{mg}, 0.06 \mathrm{mmol})$, as a yellow solid, from binol (rac)1f (31.2 mg, $0.10 \mathrm{mmol}$ ), $\mathrm{NaHCO}_{3}(85.3 \mathrm{mg}, 1.02 \mathrm{mmol}, 10$ equiv) and a solution of Oxone (124.9 mg, $0.41 \mathrm{mmol}$, 4 equiv) in Milli-Q water ( $1 \mathrm{~mL}$ ). The crude mixture was purified by preparative TLC with neutral silica gel (heptane/EtOAc 1:2).

${ }^{1} \mathrm{H}$ NMR $\left(300 \mathrm{MHz}, \mathrm{CDCl}_{3}\right) \delta: 8.10(\mathrm{t}, J=8.5 \mathrm{~Hz}, 2 \mathrm{H}), 7.73(\mathrm{~d}, J=8.8 \mathrm{~Hz}, 1 \mathrm{H}), 7.58(\mathrm{~d}, J=1.9 \mathrm{~Hz}, 1 \mathrm{H}), 7.32$ (dd, $J=8.8,1.9 \mathrm{~Hz}, 1 \mathrm{H}), 7.26(\mathrm{dd}, J=8.0,2.0 \mathrm{~Hz}, 1 \mathrm{H}), 7.10(\mathrm{~d}, J=8.7 \mathrm{~Hz}, 1 \mathrm{H}), 6.98(\mathrm{~d}, J=1.9 \mathrm{~Hz}, 1 \mathrm{H}), 6.59$ (d, $J=9.8 \mathrm{~Hz}, 1 \mathrm{H}), 6.04(\mathrm{~d}, J=9.8 \mathrm{~Hz}, 1 \mathrm{H}), 4.88(\mathrm{~s}, 1 \mathrm{H}), 3.05-2.94(\mathrm{~m}, 1 \mathrm{H}), 2.94-2.85(\mathrm{~m}, 1 \mathrm{H}), 2,63(\mathrm{~s}$, $1 \mathrm{H}), 1.30(\mathrm{~d}, J=6.9 \mathrm{~Hz}, 6 \mathrm{H}), 1.25(\mathrm{~d}, J=6.9,6 \mathrm{H})$.

${ }^{13} \mathrm{C}$ NMR (76 MHz, $\mathrm{CDCl}_{3}$ ) $\delta: 156.8,149.9,143.9,132.3,132.2,131.3,131.3,130.7,129.5,128.8,127.3$, $126.6,126.2,125.7,125.1,121.8,120.5,112.9,107.6,81.3,34.1,33.9,24.1,24.0$ (2C), 23.9.

HRMS (ESI): calculated for $\mathrm{C}_{26} \mathrm{H}_{26} \mathrm{NaO}_{3}\left([\mathrm{M}+\mathrm{Na}]^{+}\right)$409.1774, found 409.1771 . 


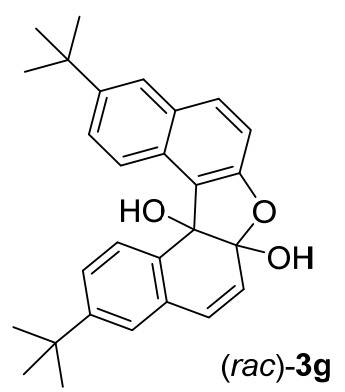

Following Method A, (rac)-3g was obtained in $81 \%$ yield $(18.9 \mathrm{mg}, 0.06 \mathrm{mmol})$, as a white solid, from binol ( $\mathrm{rac}$ )$1 \mathrm{~g}$ (29.7 mg, $0.07 \mathrm{mmol}), \mathrm{NaHCO}_{3}(62.7 \mathrm{mg}, 0.7 \mathrm{mmol}, 10$ equiv) and a solution of Oxone $(91.7 \mathrm{mg}, 0.30 \mathrm{mmol}, 4$ equiv) in Milli-Q water $(0.75 \mathrm{~mL})$. The crude mixture was purified by flash chromatography with neutral silica gel $\left(\mathrm{CH}_{2} \mathrm{Cl}_{2} / \mathrm{EtOAc} 10: 1\right)$.

${ }^{1} \mathrm{H}$ NMR $\left(300 \mathrm{MHz}, \mathrm{CDCl}_{3}\right)$ 8: $8.12(\mathrm{~m}, 2 \mathrm{H}), 7.75(\mathrm{~d}, J=8.8 \mathrm{~Hz}, 1 \mathrm{H}), 7.72-7.68(\mathrm{~m}, 1 \mathrm{H}), 7.51(\mathrm{dd}, J=8.9,2.0$ $\mathrm{Hz}, 1 \mathrm{H}), 7.43(\mathrm{dd}, J=8.2,2.0 \mathrm{~Hz}, 1 \mathrm{H}), 7.15-7.06(\mathrm{~m}, 2 \mathrm{H}), 6.60(\mathrm{~d}, J=9.9 \mathrm{~Hz}, 1 \mathrm{H}), 6.04(\mathrm{~d}, J=9.8 \mathrm{~Hz}, 1 \mathrm{H})$, $4.87(\mathrm{~s}, 1 \mathrm{H}), 2.78(\mathrm{~s}, 1 \mathrm{H}), 1.37(\mathrm{~s}, 9 \mathrm{H}), 1.31(\mathrm{~s}, 9 \mathrm{H})$.

${ }^{13} \mathrm{C}$ NMR (75 MHz, CDCl 3 ) 8: 156.8, 152.1, 145.9, 132.4, 131.8, 131.3, 130.8, 130.4, 128.9, 128.4, 126.2, $125.4,125.0,124.8,124.2,121.4,120.2,112.7,107.4,81.0,34.6(2 \mathrm{C}), 31.2(6 \mathrm{C})$.

HRMS (ESI): calculated for $\mathrm{C}_{28} \mathrm{H}_{30} \mathrm{O}_{3} \mathrm{Na}\left([\mathrm{M}+\mathrm{Na}]^{+}\right)$437.2087, found 437.2071.

Chiral HPLC: Daicel Chiralpak IC, $n$-hexane / 2-propanol: 90/10, flow rate: $1.0 \mathrm{~mL} / \mathrm{min}, \lambda: 210 \mathrm{~nm}, t_{R}: 12.62 \mathrm{~min}$, $t_{R}: 14.53 \mathrm{~min}, \mathrm{~T}: 25^{\circ} \mathrm{C}$.

(6aR,13cS)-3,11-Di-tert-butyldinaphtho[2,1-b:1',2'-d]furan-6a,13c-diol (3g)

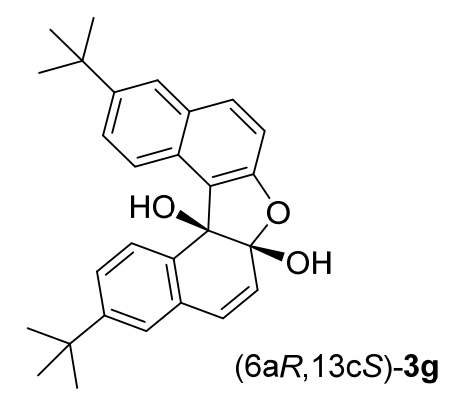


Following Method A, (6aR,13cS)-3g was obtained in $53 \%$ yield $(22.5 \mathrm{mg}, 0.05 \mathrm{mmol})$, as a white solid, from binol (R)-1g (ee 97\%) (40.5 mg, $0.12 \mathrm{mmol}$ ), $\mathrm{NaHCO}_{3}(85.4 \mathrm{mg}, 1.2 \mathrm{mmol}, 10$ equiv) and a solution of Oxone (125.0 mg, $0.48 \mathrm{mmol}, 4$ equiv) in Milli- $\mathrm{Q}$ water $(1.02 \mathrm{~mL})$. The crude mixture was purified by flash chromatography with neutral silica gel $\left(\mathrm{CH}_{2} \mathrm{Cl}_{2} /\right.$ EtOAc 10:1).

$[\alpha]_{\mathrm{D}}{ }^{20}=+106.2\left(\mathrm{c} 0.016, \mathrm{CHCl}_{3}\right)$.

Chiral HPLC: Daicel Chiralpak IC, $n$-hexane / 2-propanol: 90/10, flow rate: $1.0 \mathrm{~mL} / \mathrm{min}, \lambda: 210 \mathrm{~nm}, t_{R}$ (major): 12.82 $\min , t_{R}(\operatorname{minor}): 14.83 \min , \mathrm{T}: 25^{\circ} \mathrm{C}, \mathbf{9 4 \%} \boldsymbol{e e}$.
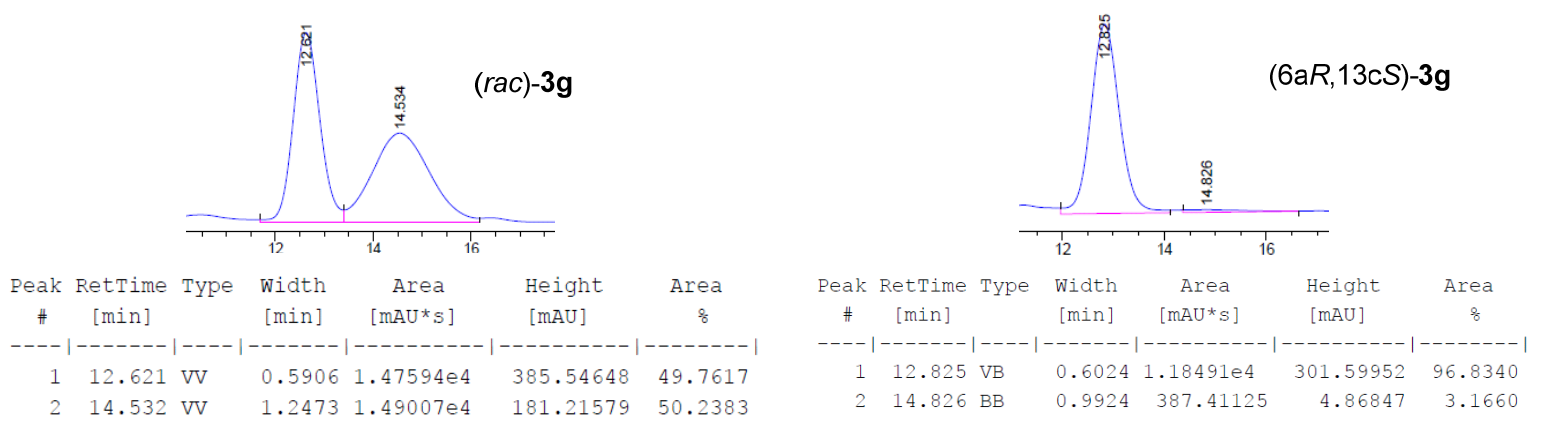

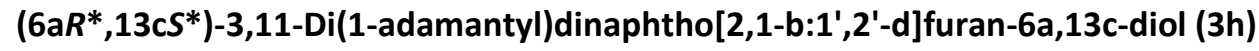

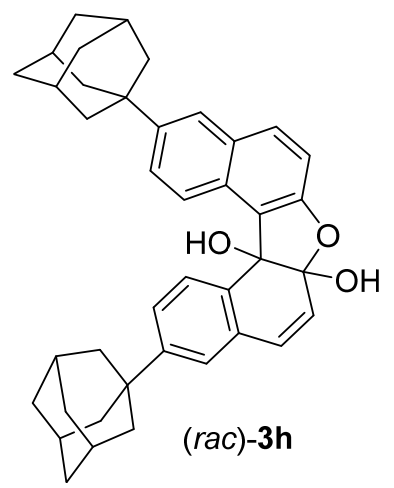

Following Method A, (rac)-3h was obtained in $52 \%$ yield $(10.7 \mathrm{mg}, 0.02 \mathrm{mmol})$, as a brown solid, from binol (rac)1h (20.0 mg, $0.04 \mathrm{mmol}), \mathrm{NaHCO}_{3}(30.3 \mathrm{mg}, 0.36 \mathrm{mmol}, 10$ equiv) and a solution of Oxone (44.3 mg, $0.14 \mathrm{mmol}$, 4 equiv) in Milli-Q water $(0.36 \mathrm{~mL})$. To dissolve all the starting material, it was necessary to add another $0.46 \mathrm{~mL}$ of acetone before the addition of the aqueous solution of Oxone. The crude mixture was purified by flash chromatography with neutral silica gel (heptane/EtOAc 3:1). 
${ }^{1} \mathrm{H}$ NMR (300 MHz, CDCl 3$)$ 8: $8.12(\mathrm{~m}, 2 \mathrm{H}), 7.75(\mathrm{~d}, J=8.9 \mathrm{~Hz}, 1 \mathrm{H}), 7.65(\mathrm{bs}, 1 \mathrm{H}), 7.50(\mathrm{~d}, J=8.6 \mathrm{~Hz}, 1 \mathrm{H})$, $7.40(\mathrm{~d}, J=8.1 \mathrm{~Hz}, 1 \mathrm{H}), 7.11(\mathrm{~m}, 2 \mathrm{H}), 6.60(\mathrm{~d}, J=9.9 \mathrm{~Hz}, 1 \mathrm{H}), 6.03(\mathrm{~d}, J=9.9 \mathrm{~Hz}, 1 \mathrm{H}), 4.87(\mathrm{~s}, 1 \mathrm{H}), 2.56(\mathrm{~s}$, $1 \mathrm{H}), 2.12$ (bs, 6H), 2.00-1.90 (bs, 12H), 1.79 (bs, 12H).

${ }^{13} \mathrm{C}$ NMR (75 MHz, CDCl 3 ) 8: 156.8, 152.1, 146.1, 132.2, 132.1, 131.3, 130.8, 130.5, 129.1, 128.5, 125.5, 124.9, 124.8, 124.6, 124.1, 121.5, 120.4, 112.6, 107.5, 80.9, 43.1 (3C), 43.0 (3C), 36.8 (3C), 36.7 (3C), 36.1 (2C), $29.0(3 C), 28.9(3 C)$.

HRMS (MALDI): calculated for $\mathrm{C}_{40} \mathrm{H}_{42} \mathrm{NaO}_{3}\left([\mathrm{M}+\mathrm{Na}]^{+}\right)$593.3134, found 593.3144.

Chiral HPLC: Daicel Chiralpak IC, $n$-hexane / 2-propanol: 90/10, flow rate: $1.0 \mathrm{~mL} / \mathrm{min}, \lambda: 254 \mathrm{~nm}, t_{R}$ (minor): 7.11 $\min , t_{R}$ (major): $11.22 \mathrm{~min}, \mathrm{~T}: 25^{\circ} \mathrm{C}$.

(6aR,13cS)-3,11-Di(1-adamantyl)dinaphtho[2,1-b:1',2'-d]furan-6a,13c-diol (3h)

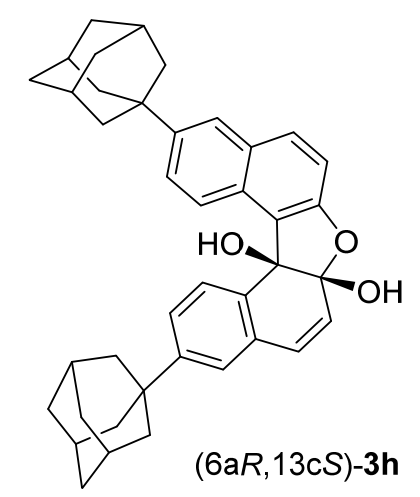

Following Method A, $(6 \mathrm{a} R, 13 \mathrm{cS})$-3h was obtained in $63 \%$ yield $(25.8 \mathrm{mg}, 0.05 \mathrm{mmol})$, as a brown solid, from binol (R)-1h (ee 99\%) (40.7 mg, $0.07 \mathrm{mmol}$ ), $\mathrm{NaHCO}_{3}(92.5 \mathrm{mg}, 1.10 \mathrm{mmol}, 15$ equiv) and a solution of Oxone (135.4 $\mathrm{mg}, 0.44 \mathrm{mmol}, 6$ equiv) in Milli- $\mathrm{Q}$ water $(0.73 \mathrm{~mL})$. To dissolve all the starting material, it was necessary to add another $0.73 \mathrm{~mL}$ of acetone before the addition of the aqueous solution of $O x$ xone. The crude mixture was purified by flash chromatography with neutral silica gel $\left(\mathrm{CH}_{2} \mathrm{Cl}_{2} /\right.$ EtOAc 1:1).

$[\alpha]_{D}^{20}=+62.5\left(\mathrm{c} 0.016, \mathrm{CHCl}_{3}\right)$.

Chiral HPLC: Daicel Chiralpak IC, $n$-hexane / 2-propanol: 90/10, flow rate: $1.0 \mathrm{~mL} / \mathrm{min}, \lambda: 254 \mathrm{~nm}, t_{R}$ (minor): 8.37 $\min , t_{R}$ (major): $12.66 \mathrm{~min}, \mathrm{~T}: 25^{\circ} \mathrm{C}, 95 \%$ ee. 

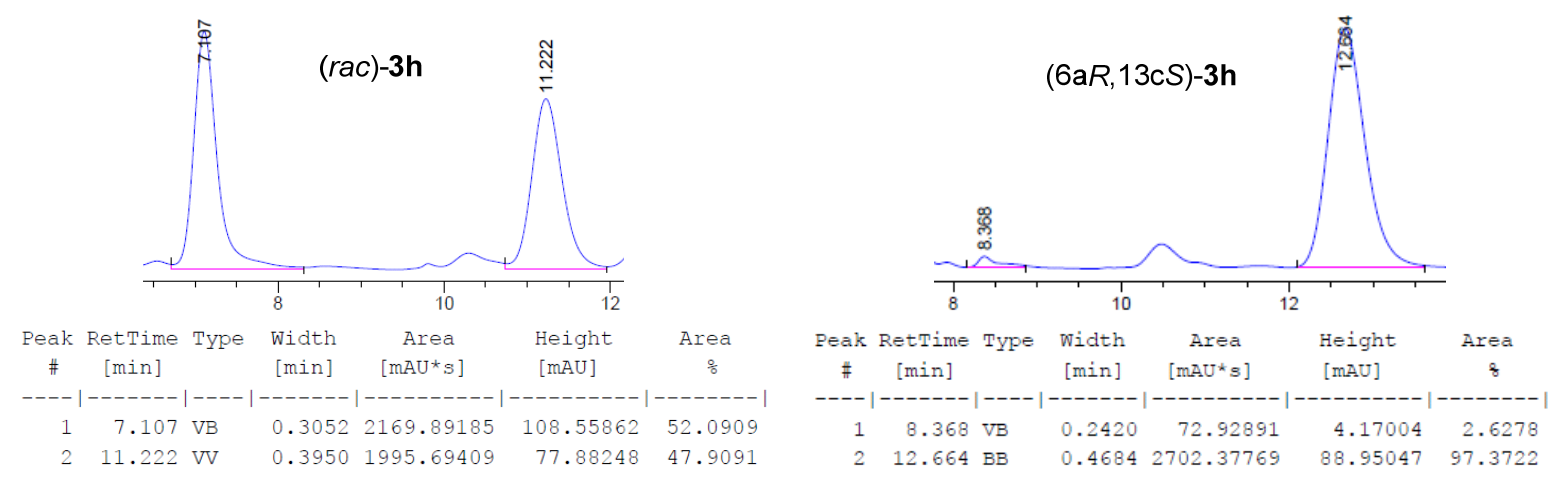

$\left(6 a R^{*}, 13 c S^{*}\right)-3,11-D i p h e n y l-6 a, 13 c-d i h y d r o d i n a p h t h o\left[2,1-b: 1^{\prime}, 2^{\prime}-d\right]$ furan-6a,13c-diol (3i)

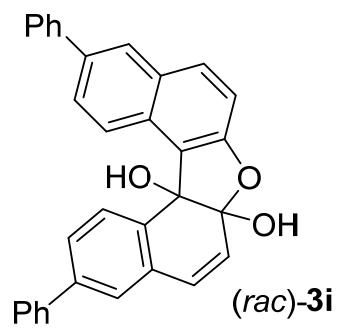

Following Method A, cis-diol ( $r a c)$-3i was obtained in $59 \%$ yield ( $12.1 \mathrm{mg}, 0.03 \mathrm{mmol}$ ), as a pale brown solid, from binol (rac)-1i (19.9 mg, $0.05 \mathrm{mmol}$ ), $\mathrm{NaHCO}_{3}$ (37.8 mg, $0.5 \mathrm{mmol}, 10$ equiv) and a solution of Oxone (55.3 mg, 0.20 mmol, 4 equiv) in Milli-Q water $(0.5 \mathrm{~mL})$. To dissolve all the starting material, it was necessary to add another $0.4 \mathrm{~mL}$ of acetone before the addition of the aqueous solution of $O x$ xone. The crude mixture was purified by flash chromatography with neutral silica gel (heptane/EtOAc 4:1).

${ }^{1} \mathrm{H} \mathrm{NMR}\left(300 \mathrm{MHz}, \mathrm{CDCl}_{3}\right) \delta: 8.32(\mathrm{~d}, J=8.0 \mathrm{~Hz}, 1 \mathrm{H}), 8.20(\mathrm{~d}, J=8.9 \mathrm{~Hz}, 1 \mathrm{H}), 7.99(\mathrm{~s}, 1 \mathrm{H}), 7.86(\mathrm{~d}, J=8.8$ $\mathrm{Hz}, 1 \mathrm{H}), 7.71-7.58(\mathrm{~m}, 6 \mathrm{H}), 7.46(\mathrm{t}, J=7.5 \mathrm{~Hz}, 5 \mathrm{H}), 7.40-7.35(\mathrm{~m}, 2 \mathrm{H}), 7.18(\mathrm{~d}, J=8.8 \mathrm{~Hz}, 1 \mathrm{H}), 6.69(\mathrm{~d}, J$ $=9.7 \mathrm{~Hz}, 1 \mathrm{H}), 6.12(\mathrm{~d}, J=9.8 \mathrm{~Hz}, 1 \mathrm{H}), 4.95(\mathrm{~s}, 1 \mathrm{H}), 2.73(\mathrm{~s}, 1 \mathrm{H})$.

${ }^{13} \mathrm{C}$ NMR (75 MHz, $\mathrm{CDCl}_{3}$ ) 8: 157.5, 142.1, 140.8, 140.2, 136.3, 133.6, 133.2, 131.8, 131.1, 130.7, 130.0, $129.2,129.0$ (5C), 127.9, 127.4, 127.2 (3C), 127.2 (3C), 126.7, 125.5, 122.1, 120.4, 113.4, 107.8, 81.1.

HRMS (ESI): calculated for $\mathrm{C}_{32} \mathrm{H}_{22} \mathrm{O}_{3} \mathrm{Na}\left([\mathrm{M}+\mathrm{Na}]^{+}\right)$477.1461, found 477.1481 .

Chiral HPLC: Daicel Chiralpak IC, $n$-hexane / 2-propanol: 90/10, flow rate: $1.0 \mathrm{~mL} / \mathrm{min}, \lambda: 254 \mathrm{~nm}, t_{R}: 29.29 \mathrm{~min}$, $t_{R}: 33.84 \min , \mathrm{T}: 25^{\circ} \mathrm{C}$. 
(6aR,13cS)-3,11-Diphenyl-6a,13c-dihydrodinaphtho[2,1-b:1',2'-d]furan-6a,13c-diol (3i)

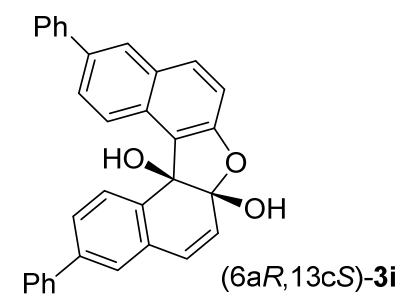

Following Method B, cis-diol (6aR,13cS)-3i was obtained in $58 \%$ yield $(5.8 \mathrm{mg}, 0.01 \mathrm{mmol})$, as a pale brown solid, from binol (R)-1i (ee >99\%) (9.7 mg, $0.02 \mathrm{mmol}), \mathrm{NaHCO}_{3}(18.5 \mathrm{mg}, 0.22 \mathrm{mmol}, 10$ equiv) and a solution of Oxone (27.0 mg, $0.09 \mathrm{mmol}, 4$ equiv) in Milli-Q water $(0.2 \mathrm{~mL}$ ). To dissolve all the starting material, it was necessary to add another $0.2 \mathrm{~mL}$ of acetone before the addition of the aqueous solution of Oxone. The crude mixture was purified by flash chromatography with neutral silica gel (heptane/EtOAc 4:1).

$[\alpha]_{\mathrm{D}}{ }^{20}=-120.5\left(c=0.013, \mathrm{CHCl}_{3}\right)$.

Chiral HPLC: Daicel Chiralpak IC, $n$-hexane / 2-propanol: 90/10, flow rate: $1.0 \mathrm{~mL} / \mathrm{min}, \lambda: 254 \mathrm{~nm}, t_{R}: 29.34 \mathrm{~min}$., $\mathrm{T}: 25^{\circ} \mathrm{C}, e e>99 \%$.
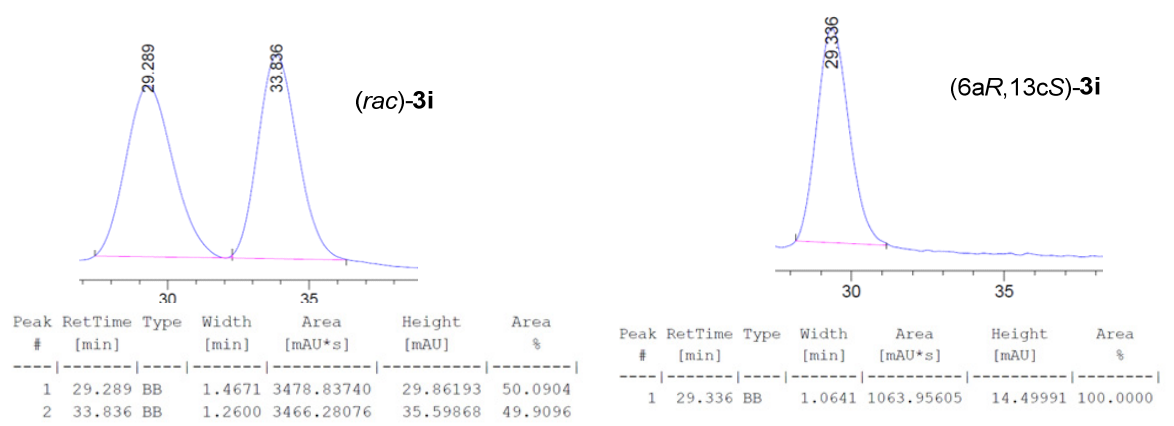

$\left(6 a R^{*}, 13 c S^{*}\right)-3,11-D i[3,5-b i s(t r i f l u o r o m e t h y l)$ phenyl]dinaphtho[2,1-b:1',2'-d]furan-6a,13c-diol (3j)

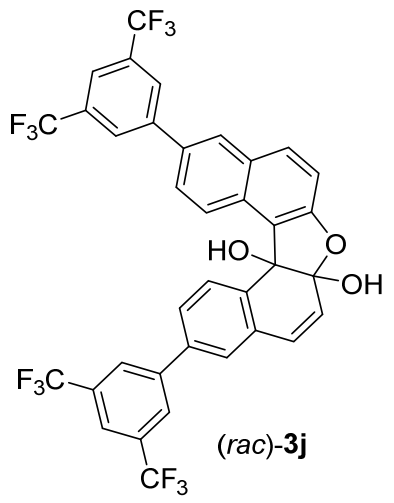


Following Method A, ( $\mathrm{rac}$ )-3j was obtained in $63 \%$ yield $(12.0 \mathrm{mg}, 0.02 \mathrm{mmol})$, as a pale yellow solid, from binol (rac)-1j (20.0 mg, $0.03 \mathrm{mmol}$ ), $\mathrm{NaHCO}_{3}(23.5 \mathrm{mg}, 0.28 \mathrm{mmol}, 10$ equiv) and a solution of Oxone (34.4 mg, 0.11 mmol, 4 equiv) in Milli-Q water $(0.28 \mathrm{~mL})$. The crude mixture was purified by flash chromatography with neutral silica gel (heptane/EtOAc 3:1).

${ }^{1} \mathrm{H} \mathrm{NMR}\left(300 \mathrm{MHz}, \mathrm{CDCl}_{3}\right)$ 8: $8.42(\mathrm{~d}, J=8.0 \mathrm{~Hz}, 1 \mathrm{H}), 8.19(\mathrm{~d}, J=8.9 \mathrm{~Hz}, 1 \mathrm{H}), 8.06$ (bs, $\left.2 \mathrm{H}\right), 8.04(\mathrm{bs}, 3 \mathrm{H})$, $7.94(\mathrm{~d}, J=8.9 \mathrm{~Hz}, 1 \mathrm{H}), 7.88(\mathrm{~d}, J=10.5 \mathrm{~Hz}, 2 \mathrm{H}), 7.69(\mathrm{~m}, 2 \mathrm{H}), 7.39(\mathrm{~d}, J=2.0 \mathrm{~Hz}, 1 \mathrm{H}), 7.25(\mathrm{~d}, J=8.7 \mathrm{~Hz}$, $1 \mathrm{H}), 6.73(\mathrm{~d}, J=9.9 \mathrm{~Hz}, 1 \mathrm{H}), 6.18(\mathrm{~d}, J=9.9 \mathrm{~Hz}, 1 \mathrm{H}), 4.94(\mathrm{~s}, 1 \mathrm{H}), 2.84(\mathrm{~s}, 1 \mathrm{H})$.

${ }^{13} \mathrm{C} \mathrm{NMR}\left(75 \mathrm{MHz}, \mathrm{CDCl}_{3}\right)$ 8: 158.2, 142.7, 142.1, 139.1, 135.2, 133.5, 133.1, 132.5, $132.4(2 \mathrm{C}, \mathrm{q}, \mathrm{J}=30 \mathrm{~Hz})$, $132.3(2 \mathrm{C}, \mathrm{q}, J=30 \mathrm{~Hz}), 130.5,130.4,129.5,127.8,127.1,127.1,127.1(2 \mathrm{C}), 127.0(2 \mathrm{C}), 126.5,126.4,126.1$, $123.4(2 \mathrm{C}, \mathrm{q}, J=270 \mathrm{~Hz}), 123.3(2 \mathrm{C}, \mathrm{q}, J=270 \mathrm{~Hz}), 122.5,121.4,120.9,120.3,113.9,107.8,80.7$.

${ }^{19}$ F NMR (282 MHz, $\left.\mathrm{CDCl}_{3}\right)$ 8: - 62.8.

HRMS (MALDI): calculated for $\mathrm{C}_{36} \mathrm{H}_{18} \mathrm{~F}_{12} \mathrm{NaO}_{3}\left([\mathrm{M}+\mathrm{Na}]^{+}\right)$749.1064, found 749.1072.

Chiral HPLC: Daicel Chiralpak IC, $n$-hexane / 2-propanol: 95/5, flow rate: $0.3 \mathrm{~mL} / \mathrm{min}, \lambda: 210 \mathrm{~nm}, t_{R}: 30.50 \mathrm{~min}, t_{R}$ : $34.34 \mathrm{~min}, \mathrm{~T}: 25^{\circ} \mathrm{C}$.

(6aR,13cS)-3,11-Bis[3,5-bis(trifluoromethyl)phenyl]dinaphtho[2,1-b:1',2'-d]furan-6a,13c-diol (3j)

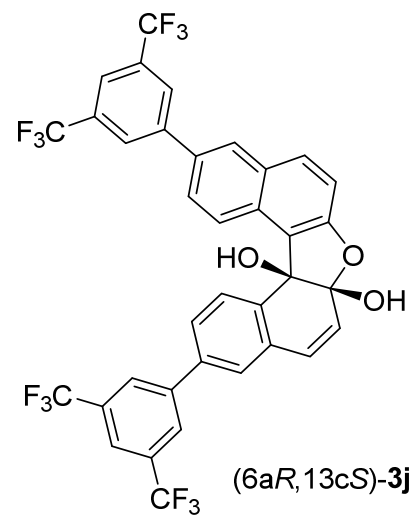

Following Method B ( 0 ㄷ for $1 \mathrm{~h}$ and $\mathrm{rt}$ for $2 \mathrm{~h}$ ), (6aR,13cS)-3j was obtained in $59 \%$ yield (13.0 $\mathrm{mg}, 0.02 \mathrm{mmol})$, as a pale yellow solid, from binol (R)-1j (20.0 mg, $0.03 \mathrm{mmol}), \mathrm{NaHCO}_{3}(35.3 \mathrm{mg}, 0.42 \mathrm{mmol}, 15$ equiv) and a solution of Oxone ( $50.7 \mathrm{mg}, 0.16 \mathrm{mmol}, 6$ equiv) in Milli-Q water $(0.28 \mathrm{~mL})$. The crude mixture was purified by flash chromatography with neutral silica gel (heptane/EtOAc 2:1). 
$[\alpha]_{\mathrm{D}}{ }^{20}=+88.2\left(\mathrm{c} 0.017, \mathrm{CHCl}_{3}\right)$.

Chiral HPLC: Daicel Chiralpak IC, $n$-hexane / 2-propanol: 95/5, flow rate: $0.3 \mathrm{~mL} / \mathrm{min}, \lambda: 210 \mathrm{~nm}, t_{R}: 30.13 \mathrm{~min}, \mathrm{~T}$ : $25^{\circ} \mathrm{C},>99 \%$ ee.
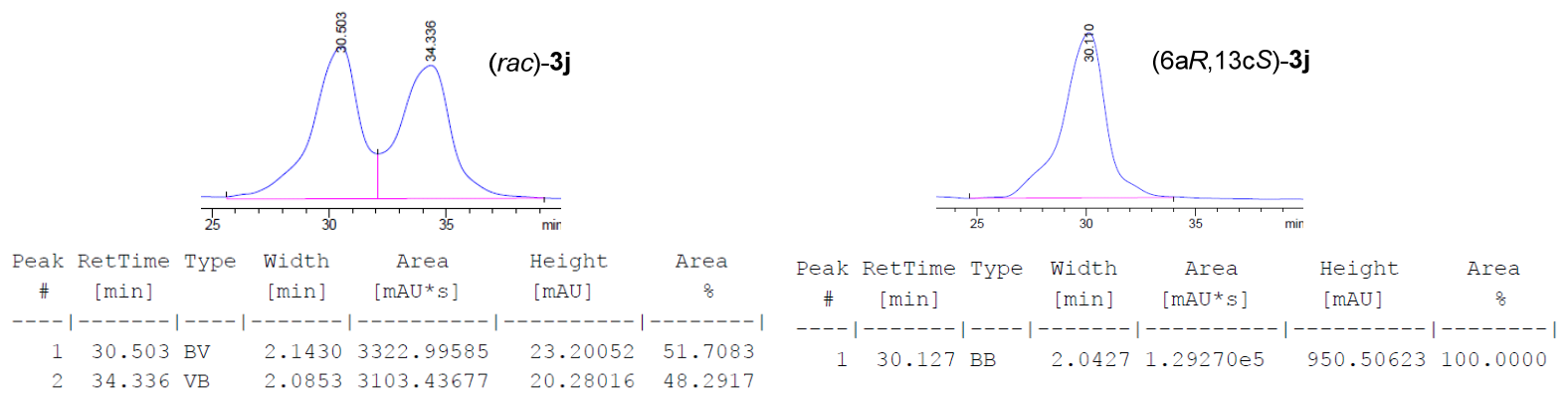

$\left(6 a R^{*}, 13 c S^{*}\right)-3,11-B i s($ phenylethynyl)dinaphtho[2,1-b:1',2'-d]furan-6a,13c-diol (3k)

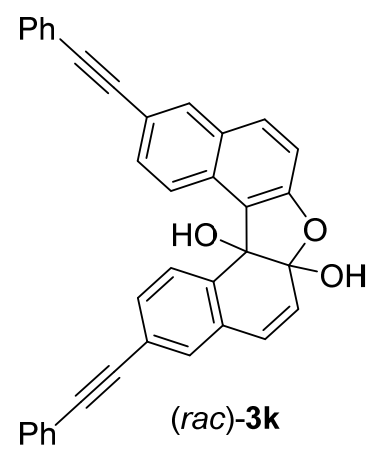

Following Method A, ( $r a c)-3 k$ was obtained in $82 \%$ yield ( $17.0 \mathrm{mg}, 0.03 \mathrm{mmol}$ ), as a yellow solid, from binol (rac)$1 \mathrm{k}$ (20.0 mg, $0.04 \mathrm{mmol}), \mathrm{NaHCO}_{3}(34.6 \mathrm{mg}, 0.41 \mathrm{mmol}, 10$ equiv) and a solution of Oxone (50.6 mg, $0.16 \mathrm{mmol}$, 4 equiv) in Milli-Q water $(0.4 \mathrm{~mL})$. To dissolve all starting material, it was necessary to add another $0.2 \mathrm{~mL}$ of acetone before the addition of the aqueous solution of Oxone. The crude mixture was purified by flash chromatography with neutral silica gel $\left(\mathrm{CH}_{2} \mathrm{Cl}_{2} / \mathrm{EtOAc} 30: 1\right)$.

${ }^{1} \mathrm{H}$ NMR $\left(300 \mathrm{MHz}, \mathrm{CDCl}_{3}\right)$ 8: $8.22(\mathrm{~d}, J=8.1 \mathrm{~Hz}, 1 \mathrm{H}), 8.03-7.96(\mathrm{~m}, 2 \mathrm{H}), 7.80(\mathrm{~d}, J=8.9 \mathrm{~Hz}, 1 \mathrm{H}), 7.61(\mathrm{dd}$, $J=8.0,1.7 \mathrm{~Hz}, 1 \mathrm{H}), 7.58-7.53(\mathrm{~m}, 4 \mathrm{H}), 7.49(\mathrm{dd}, J=8.9,1.6 \mathrm{~Hz}, 1 \mathrm{H}), 7.40-7.34(\mathrm{~m}, 6 \mathrm{H}), 7.32(\mathrm{~d}, J=1.7$ $\mathrm{Hz}, 1 \mathrm{H}), 7.18(\mathrm{~d}, J=8.8 \mathrm{~Hz}, 1 \mathrm{H}), 6.62(\mathrm{~d}, J=9.9 \mathrm{~Hz}, 1 \mathrm{H}), 6.11(\mathrm{~d}, J=9.8 \mathrm{~Hz}, 1 \mathrm{H}), 4.93(\mathrm{~s}, 1 \mathrm{H}), 2.72(\mathrm{~s}, 1 \mathrm{H})$. 
${ }^{13} \mathrm{C} \mathrm{NMR}\left(75 \mathrm{MHz}, \mathrm{CDCl}_{3}\right.$ ) 8: 158.0, 134.3, 132.8, 132.6, 131.7 (2C), 131.6 (2C), 131.5, 131.4, 130.7, 130.5, $130.2,130.1,130.0,128.6,128.5,128.4$ (2C), 128.4 (2C), 128.3, 125.6, 124.3, 123.3, 122.9, 121.4, 120.4, $118.3,113.5,107.7,90.6,89.6,89.4,88.5,80.8$.

HRMS (MALDI): calculated for $\mathrm{C}_{36} \mathrm{H}_{22} \mathrm{NaO}_{3}\left([\mathrm{M}+\mathrm{Na}]^{+}\right)$525.1569, found 525.1578 .

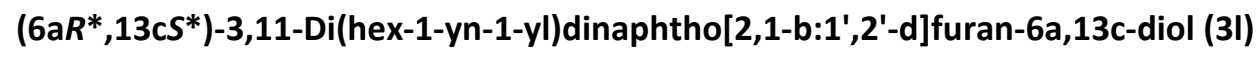

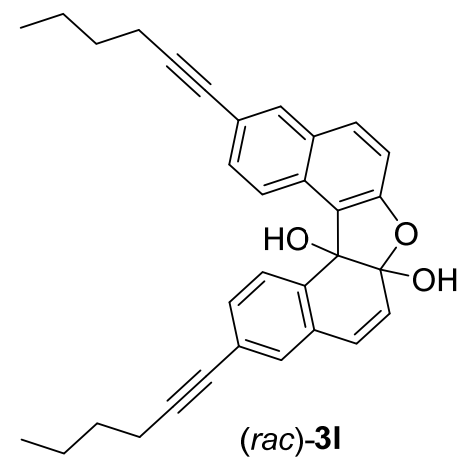

Following Method A, (rac)-3l was obtained in $56 \%$ yield (18.2 mg, $0.04 \mathrm{mmol})$, as a yellow solid, from binol ( $\mathrm{rac}$ )1 ( $\left(31.1 \mathrm{mg}, 0.07 \mathrm{mmol}\right.$ ), $\mathrm{NaHCO}_{3}(58.5 \mathrm{mg}, 0.7 \mathrm{mmol}, 10$ equiv) and a solution of Oxone ( $85.6 \mathrm{mg}, 0.28 \mathrm{mmol}, 4$ equiv) in Milli-Q water $(0.7 \mathrm{~mL})$. The crude mixture was purified by flash chromatography with neutral silica gel (heptane/EtOAc 2:1).

${ }^{1} \mathrm{H}$ NMR (300 MHz, CDCl 3$)$ 8: $8.11(\mathrm{~d}, J=8.0 \mathrm{~Hz}, 1 \mathrm{H}), 7.90(\mathrm{~d}, J=8.7 \mathrm{~Hz}, 1 \mathrm{H}), 7.81(\mathrm{~s}, 1 \mathrm{H}), 7.71(\mathrm{~d}, J=8.8$ $\mathrm{Hz}, 1 \mathrm{H}), 7.43(\mathrm{dd}, J=8.1,1.8 \mathrm{~Hz}, 1 \mathrm{H}), 7.33(\mathrm{dd}, J=8.8,1.7 \mathrm{~Hz}, 1 \mathrm{H}), 7.15(\mathrm{~d}, J=1.7 \mathrm{~Hz}, 1 \mathrm{H}), 7.12(\mathrm{~d}, J=8.8$ $\mathrm{Hz}, 1 \mathrm{H}), 6.55(\mathrm{~d}, J=9.9 \mathrm{~Hz}, 1 \mathrm{H}), 6.05(\mathrm{~d}, J=9.9 \mathrm{~Hz}, 1 \mathrm{H}), 4.88(\mathrm{~s}, 1 \mathrm{H}), 2.62(\mathrm{~s}, 1 \mathrm{H}), 2.42(\mathrm{~m}, 4 \mathrm{H}), 1.61-1.54$ $(m, 4 H), 1.54-1.44(m, 4 H), 0.95(t, J=7.2 \mathrm{~Hz}, 6 \mathrm{H})$.

${ }^{13} \mathrm{C}$ NMR (126 MHz, CDCl 3$)$ ): 157.7, 133.7, 132.6, 132.3, 131.5, 131.4, 130.9, 130.7, 130.5, 130.1, 129.8, $128.6,125.6,125.2,121.4,120.5,119.3,113.4,107.7,92.0,90.8,80.9,80.6,80.0,31.0,30.9,29.9,29.8$, $19.3,19.3,13.8,13.8$.

HRMS (ESI): calculated for $\mathrm{C}_{32} \mathrm{H}_{30} \mathrm{NaO}_{3}\left([\mathrm{M}+\mathrm{Na}]^{+}\right)$485.2087, found 485.2072. 
$\left(6 a R^{*}, 13 c S^{*}\right)-2,12-$ Dibromodinaphtho[2,1-b:1',2'-d]furan-6a,13c-diol (3n)<smiles>OC12C=Cc3ccc(Br)cc3C1(O)c1c(ccc3ccc(Br)cc13)O2</smiles>

Following Method A, (rac)-3n was obtained in $63 \%$ yield $(21.9 \mathrm{mg}, 0.05 \mathrm{mmol})$, as a yellow solid, from binol ( $\mathrm{rac}$ )1n (33.5 mg, $0.07 \mathrm{mmol}), \mathrm{NaHCO}_{3}(63.4 \mathrm{mg}, 0.75 \mathrm{mmol}, 10$ equiv) and a solution of Oxone ( $92.7 \mathrm{mg}, 0.30 \mathrm{mmol}$, 4 equiv) in Milli- $Q$ water $(0.75 \mathrm{~mL})$. The crude mixture was purified by flash chromatography with neutral silica gel (heptane/EtOAc 3:1).

${ }^{1} \mathrm{H}$ NMR $\left(300 \mathrm{MHz}, \mathrm{CDCl}_{3}\right) \delta: 8.38(\mathrm{~d}, J=2.0 \mathrm{~Hz}, 1 \mathrm{H}), 8.14(\mathrm{~d}, J=1.9 \mathrm{~Hz}, 1 \mathrm{H}), 7.76(\mathrm{~d}, J=8.8 \mathrm{~Hz}, 1 \mathrm{H}), 7.62$ (d, $J=8.7 \mathrm{~Hz}, 1 \mathrm{H}$ ), 7.50 (dd, $J=8.1,2.0 \mathrm{~Hz}, 1 \mathrm{H}$ ), 7.37 (dd, $J=8.7,1.9 \mathrm{~Hz} 1 \mathrm{H}$ ), $7.12(\mathrm{~d}, J=8.8 \mathrm{~Hz}, 1 \mathrm{H}$ ), 7.00 $(\mathrm{d}, J=8.1 \mathrm{~Hz}, 1 \mathrm{H}), 6.54(\mathrm{~d}, J=9.9 \mathrm{~Hz}, 1 \mathrm{H}), 6.04(\mathrm{~d}, J=9.9 \mathrm{~Hz}, 1 \mathrm{H}), 4.97(\mathrm{~s}, 1 \mathrm{H}), 2.83(\mathrm{~s}, 1 \mathrm{H})$.

${ }^{13} \mathrm{C}$ NMR (126 MHz, CDCl $)$ ) $8: 158.2,136.3,133.2,132.4,131.9,131.7,130.9,130.3,130.2,129.4,128.7$, $127.1,125.4,123.7,122.5,122.4,119.2,113.3,107.8,80.5$.

HRMS (ESI): calculated for $\mathrm{C}_{20} \mathrm{H}_{12} \mathrm{Br}_{2} \mathrm{NaO}_{3}\left([\mathrm{M}+\mathrm{Na}]^{+}\right)$482.9045, found 482.9040 .

\section{$\left(8 \mathrm{~b} R^{*}, 17 \mathrm{cS}{ }^{*}\right)$-Diphenanthro[9,10-b:9',10'-d]furan-8b,17c-diol (3o)}

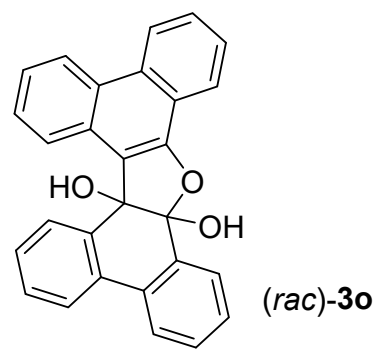

Following Method A, (rac)-3o was obtained in $66 \%$ yield $(14.6 \mathrm{mg}, 0.04 \mathrm{mmol})$, as a brown solid, from binol (rac)10 (21.4 mg, $0.055 \mathrm{mmol})(21.4 \mathrm{mg}, 0.055 \mathrm{mmol}), \mathrm{NaHCO}_{3}(46.5 \mathrm{mg}, 0.55 \mathrm{mmol}, 10$ equiv) and a solution of Oxone (68.1 mg, $0.22 \mathrm{mmol}$, 4 equiv) in Milli-Q water $(0.55 \mathrm{~mL})$. The crude mixture was purified by flash chromatography with neutral silica gel (heptane/EtOAc 3:1). 
${ }^{1} \mathrm{H} \mathrm{NMR}\left(300 \mathrm{MHz}, \mathrm{CDCl}_{3}\right) \delta: 8.57(\mathrm{~m}, 2 \mathrm{H}), 8.45(\mathrm{~d}, J=8.0 \mathrm{~Hz}, 1 \mathrm{H}), 8.27(\mathrm{~d}, J=7.8 \mathrm{~Hz}, 1 \mathrm{H}), 8.16(\mathrm{~d}, J=8.0$ $\mathrm{Hz}, 1 \mathrm{H}), 8.03(\mathrm{~d}, J=7.8 \mathrm{~Hz}, 1 \mathrm{H}), 7.85(\mathrm{dd}, \underline{J}=7.4,1.7 \mathrm{~Hz}, 1 \mathrm{H}), 7.78-7.67(\mathrm{~m}, 1 \mathrm{H}), 7.66(\mathrm{td}, J=7.3,1.6 \mathrm{~Hz}$, 2H), 7.54 (ddd, $J=9.1,7.3,1.7 \mathrm{~Hz}, 2 \mathrm{H}), 7.46-7.35(\mathrm{~m}, 2 \mathrm{H}), 7.39-7.29(\mathrm{~m}, 2 \mathrm{H}), 5.27(\mathrm{~s}, 1 \mathrm{H}), 2.70(\mathrm{~s}, 1 \mathrm{H})$.

${ }^{13} \mathrm{C}$ NMR (76 MHz, CDCl 3 ) 8: 154.5, 133.6, 133.2, 132.9, 132.4, 132.2, 130.0, 129.4, 129.3, 129.1, 128.8, $128.3,128.1,127.6,127.2,126.9,126.7,124.5,124.1,123.4,122.9,122.7,122.6,122.1,121.7,117.0$, 108.5, 81.3.

HRMS (ESI): calculated for $\mathrm{C}_{28} \mathrm{H}_{18} \mathrm{NaO}_{3}\left([\mathrm{M}+\mathrm{Na}]^{+}\right)$425.1154, found 425.1143.

$\left(6 a R^{*}, 13 c S^{*}\right)-8-(1-A d a m a n t y l)-3,11-d i b r o m o d i n a p h t h o\left[2,1-b: 1^{\prime}, 2^{\prime}-d\right] f u r a n-6 a, 13 c-d i o l ~(3 p)$

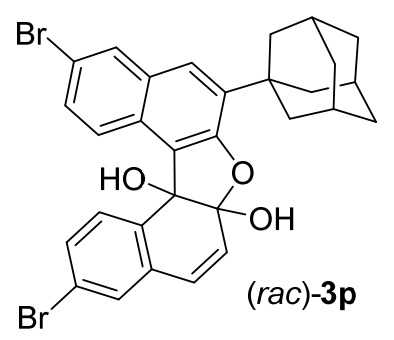

Following Method A, ( $\mathrm{rac}$ )-3p was obtained in $54 \%$ yield (10.5 mg, $0.02 \mathrm{mmol}$ ), as a pale brown solid, from binol (rac)-1p (18.9 mg, $0.03 \mathrm{mmol}$ ), $\mathrm{NaHCO}_{3}(40.1 \mathrm{mg}, 0.48 \mathrm{mmol}, 15$ equiv) and a solution of Oxone (58.7 mg, 0.19 mmol, 6 equiv) in Milli-Q water ( $0.32 \mathrm{~mL})$. To dissolve all starting material, it was necessary to add another 0.32 $\mathrm{mL}$ of acetone before the addition of the aqueous solution of Oxone. The crude mixture was purified by flash chromatography with neutral silica gel $\left(\mathrm{CH}_{2} \mathrm{Cl}_{2} / \mathrm{EtOAc} 10: 1\right)$.

${ }^{1} \mathrm{H}$ NMR $\left(300 \mathrm{MHz}, \mathrm{CDCl}_{3}\right) \delta: 8.09(\mathrm{~d}, J=8.2 \mathrm{~Hz}, 1 \mathrm{H}), 7.91(\mathrm{~d}, J=2.1 \mathrm{~Hz}, 1 \mathrm{H}), 7.77(\mathrm{~d}, J=9.2 \mathrm{~Hz}, 1 \mathrm{H}), 7.55$ (m, 2H), 7.38 (dd, J = 9.1, 2.1 Hz, 1H), 7.26 (bs, $1 \mathrm{H}), 6.53(\mathrm{~d}, J=9.8 \mathrm{~Hz}, 1 \mathrm{H}), 6.12(\mathrm{~d}, J=9.9 \mathrm{~Hz}, 1 \mathrm{H}$ ), 4.85 (s, $1 \mathrm{H}), 2,56(\mathrm{~s}, 1 \mathrm{H}), 2.12(\mathrm{~s}, 9 \mathrm{H}), 1.81(\mathrm{~s}, 6 \mathrm{H})$.

${ }^{13} \mathrm{C}$ NMR (126 MHz, CDCl 3 ) ס: 156.9, 137.2, 133.6, 133.4, 131.7, 131.1, 131.1, 130.4, 130.2, 129.9, 129.8, $127.6,127.4,126.4,123.1,122.4,120.8,117.0,106.9,80.0,40.4$ (3C), 36.8 (3C), 36.7, 28.8 (3C).

HRMS (ESI): calculated for $\mathrm{C}_{30} \mathrm{H}_{26} \mathrm{Br}_{2} \mathrm{NaO}_{3}\left([\mathrm{M}+\mathrm{Na}]^{+}\right) 617.0303$, found 617.0287 .

Chiral HPLC: Daicel Chiralpak IB, n-hexane / 2-propanol: 97/3, flow rate: $0.3 \mathrm{~mL} / \mathrm{min}, \lambda: 254 \mathrm{~nm}, \mathrm{t}_{\mathrm{R}}: 34.00$ $\min , \mathrm{t}_{\mathrm{R}}: 36.32 \mathrm{~min}, \mathrm{~T}: 25^{\circ} \mathrm{C}$. 


\section{(6aR,13cS)-8-(1-Adamantyl)-3,11-dibromodinaphtho[2,1-b:1',2'-d]furan-6a,13c-diol (3p)}

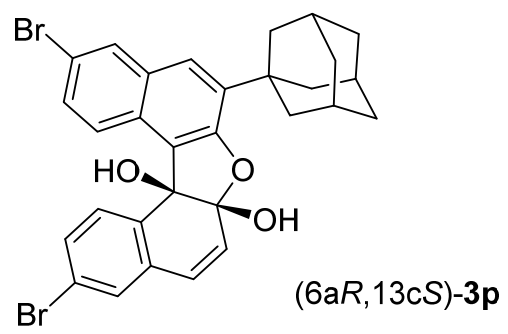

Following Method A, $(6 a R, 13 \mathrm{cS})-3 p$ was obtained in $56 \%$ yield $(10.0 \mathrm{mg}, 0.02 \mathrm{mmol})$, as a pale brown solid, from binol (R)-1p (ee >99\%) (17.8 mg, $0.03 \mathrm{mmol}), \mathrm{NaHCO}_{3}(25.2 \mathrm{mg}, 0.30 \mathrm{mmol}, 10$ equiv) and a solution of Oxone ( $36.9 \mathrm{mg}, 0.12 \mathrm{mmol}, 4$ equiv) in Milli-Q water $(0.3 \mathrm{~mL}$ ). To dissolve all starting material, it was necessary to add another $0.20 \mathrm{~mL}$ of acetone before the addition of the aqueous solution of Oxone. The crude mixture was purified by flash chromatography with neutral silica gel $\left(\mathrm{CH}_{2} \mathrm{Cl}_{2} / \mathrm{EtOAc} 10: 1\right)$.

$[\alpha]_{\mathrm{D}}{ }^{20}=-46.1\left(\mathrm{c} 0.013, \mathrm{CHCl}_{3}\right)$.

Chiral HPLC: Daicel Chiralpak IB, $n$-hexane / 2-propanol: 97/3, flow rate: $0.3 \mathrm{~mL} / \mathrm{min}, \lambda: 254 \mathrm{~nm}, t_{R}$ (major): 33.16 $\min , \mathrm{T}: 25^{\circ} \mathrm{C},>99 \%$ ee.
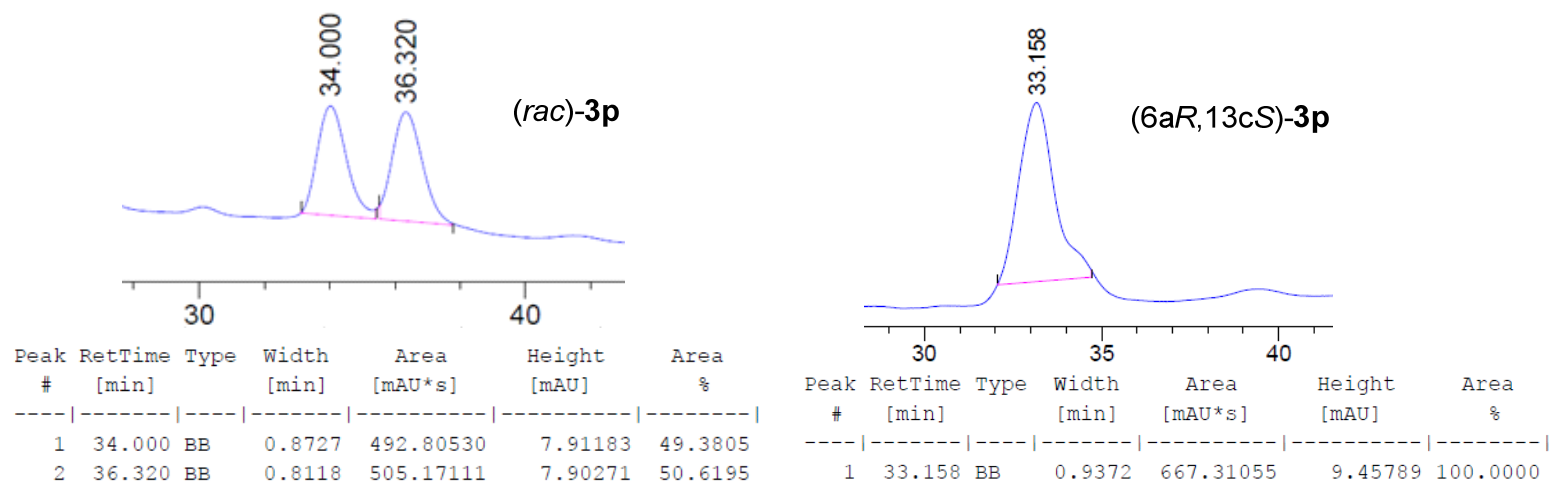


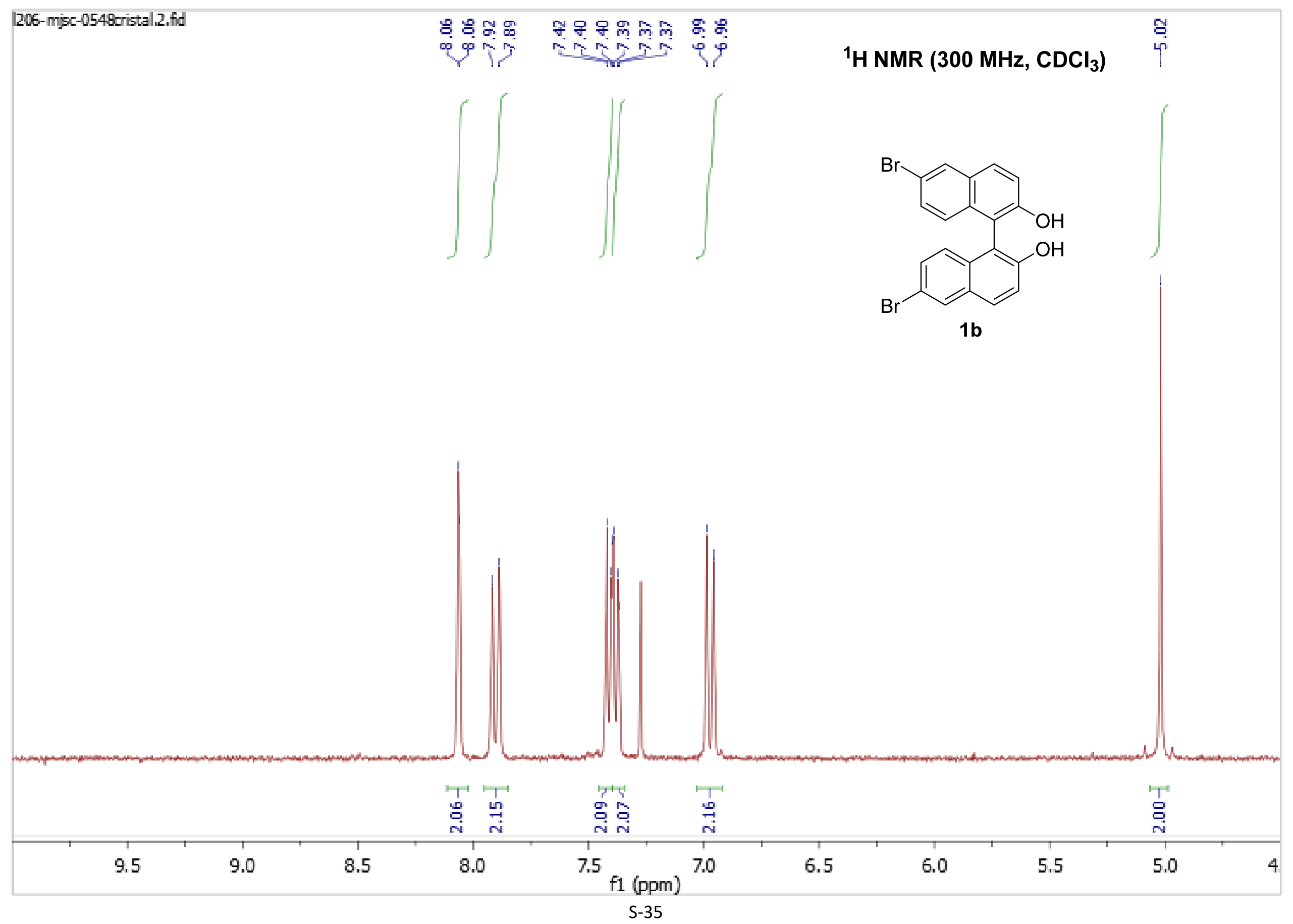




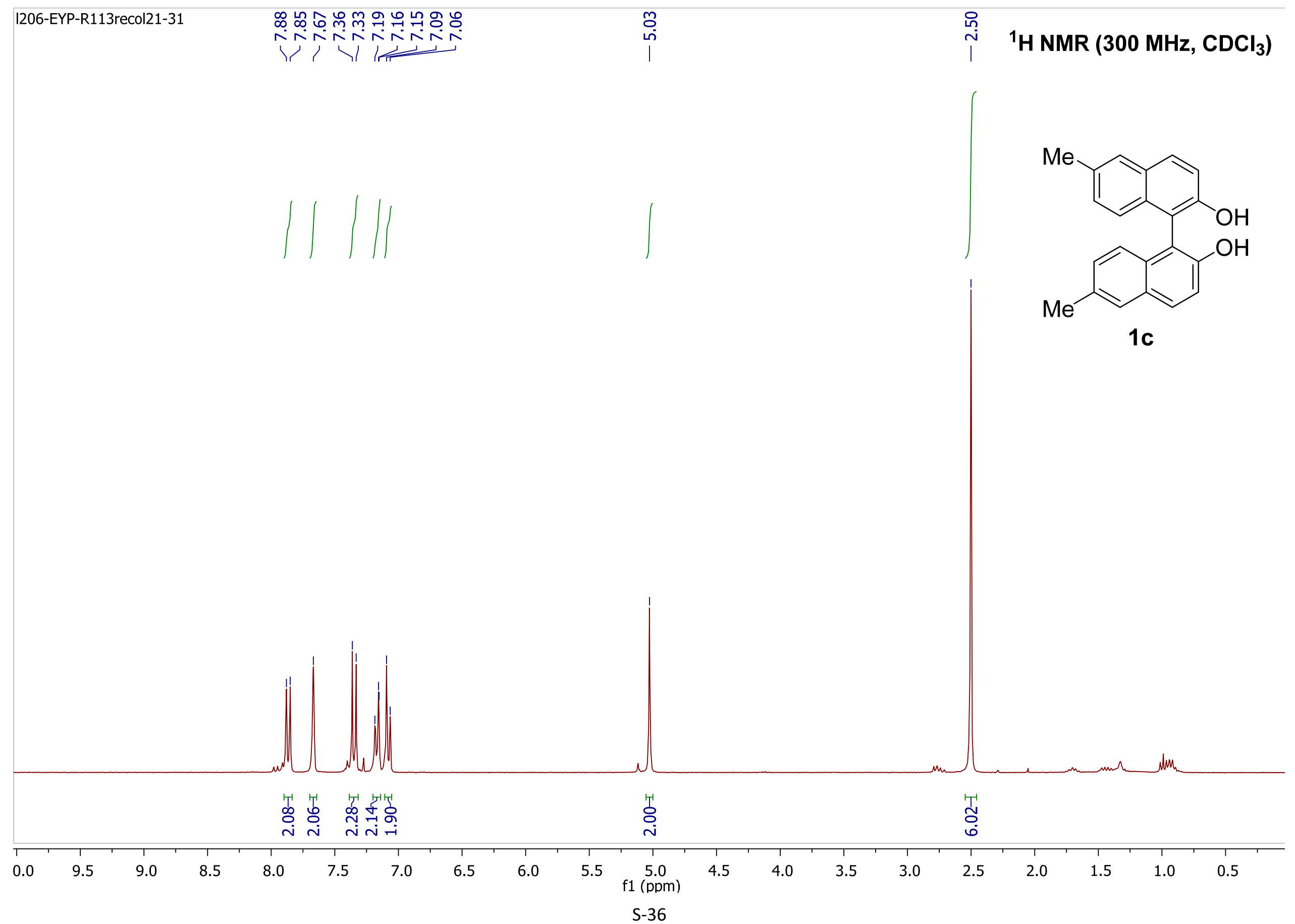




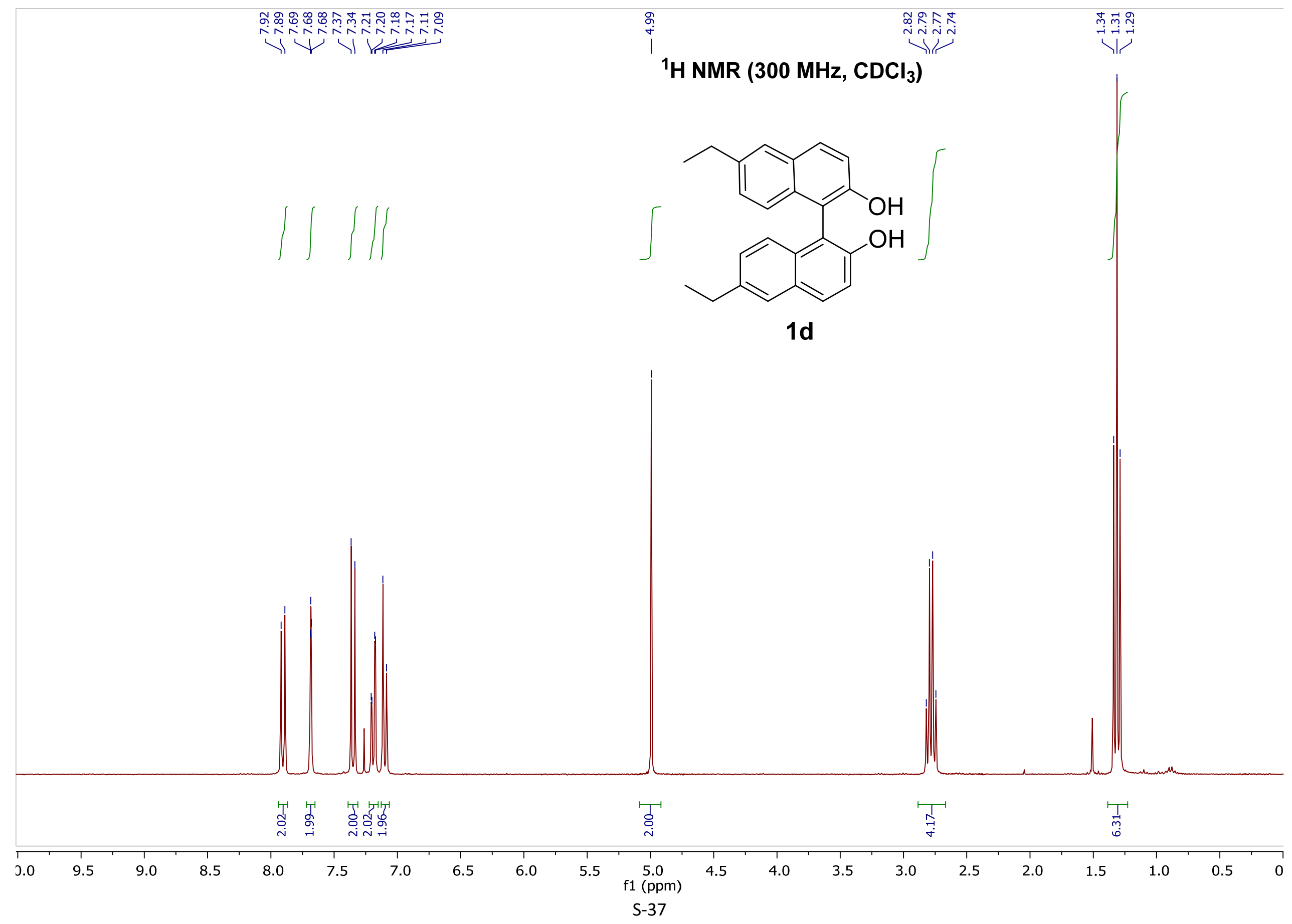




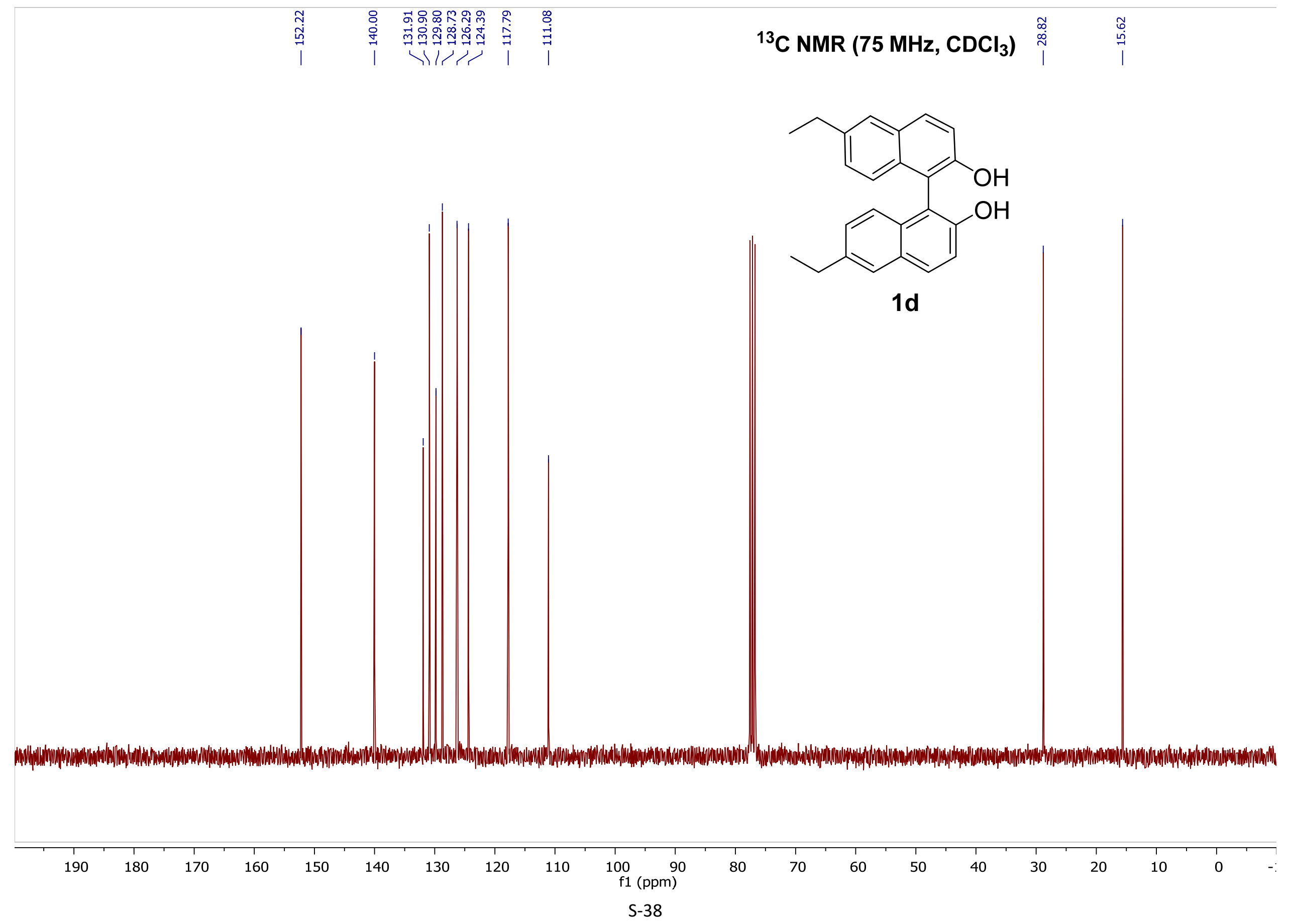




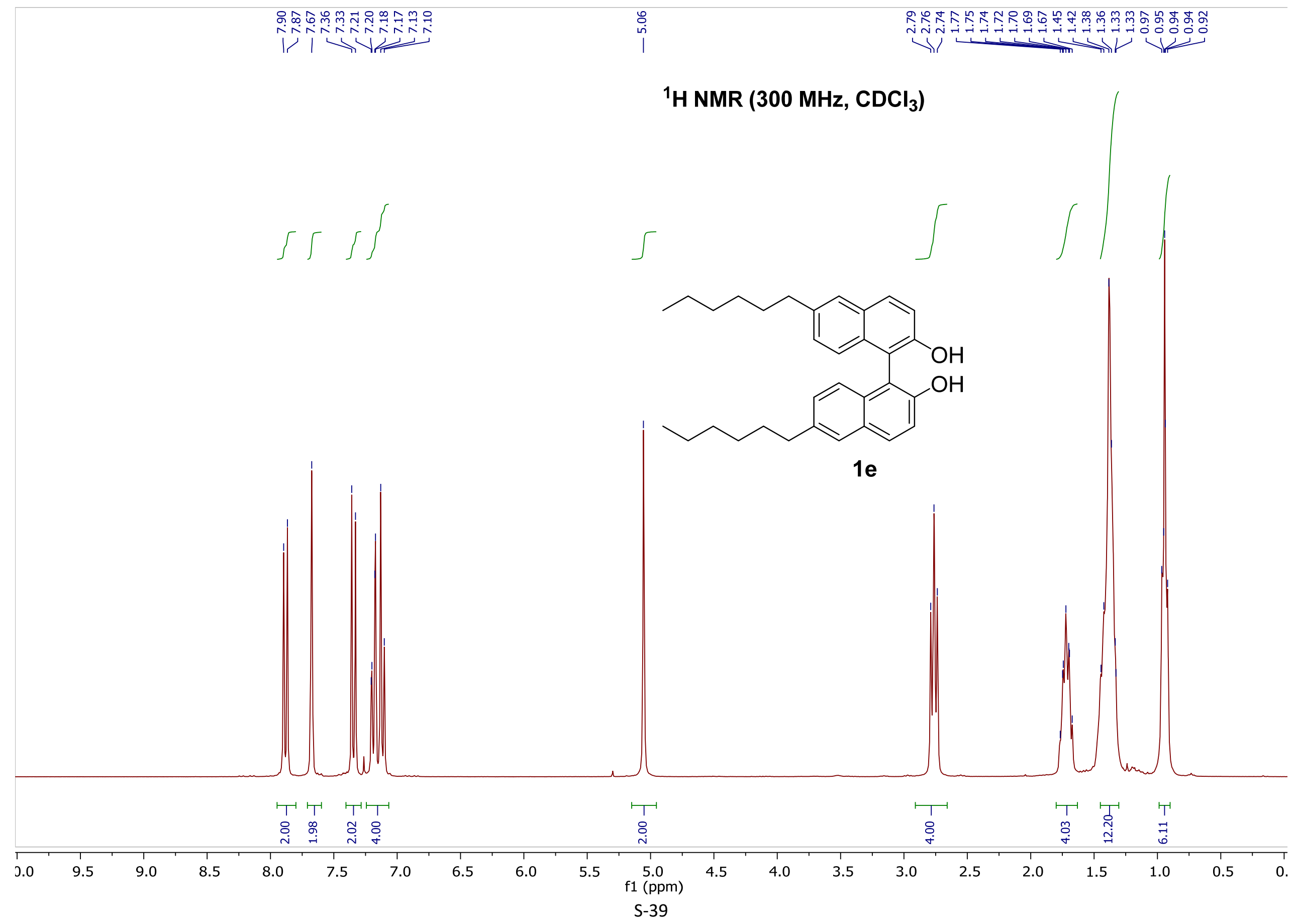




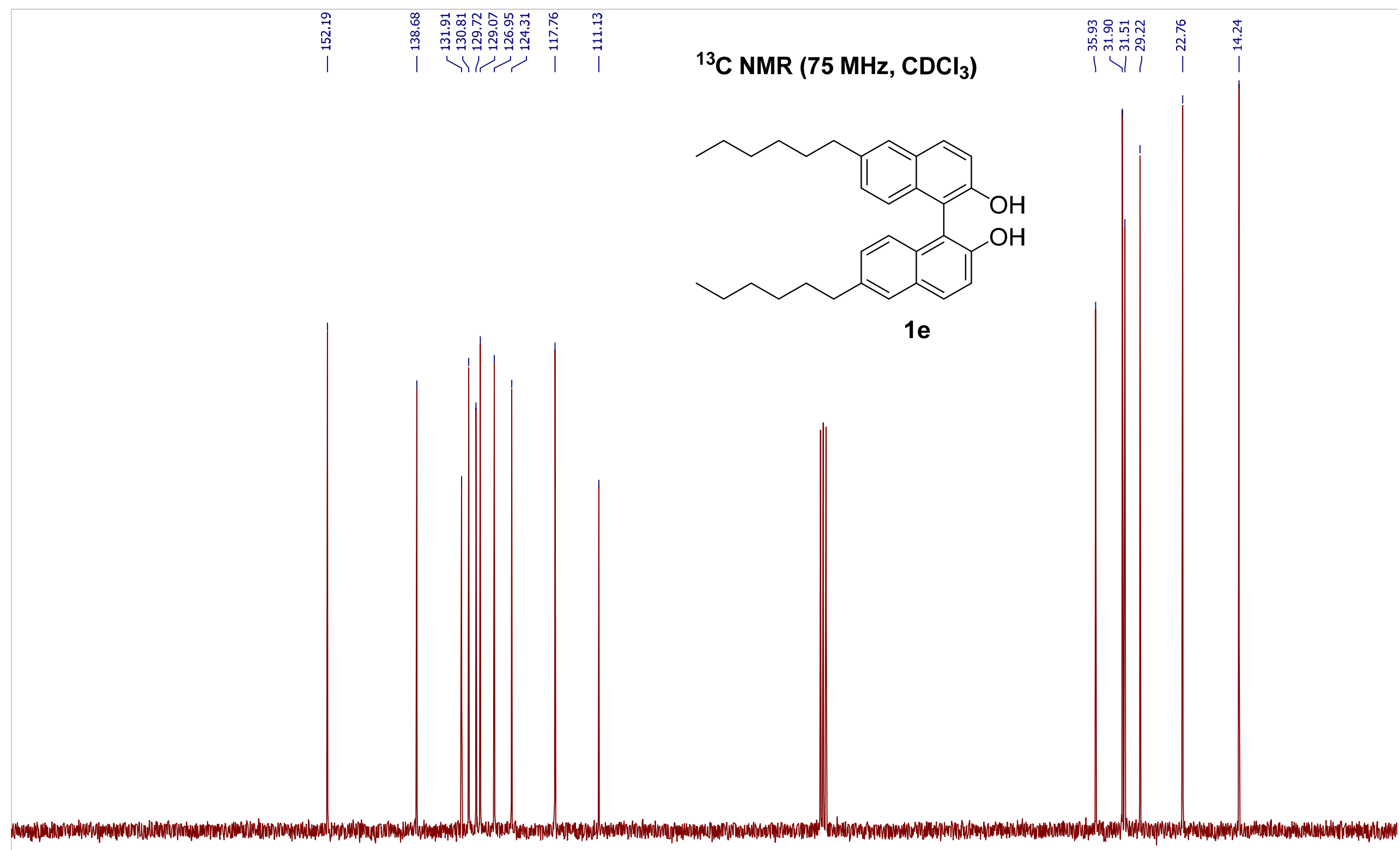

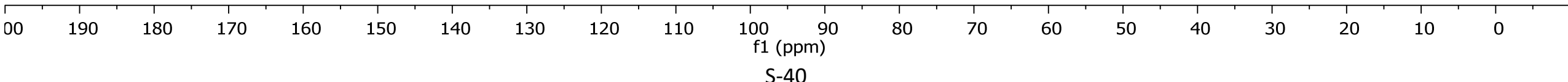




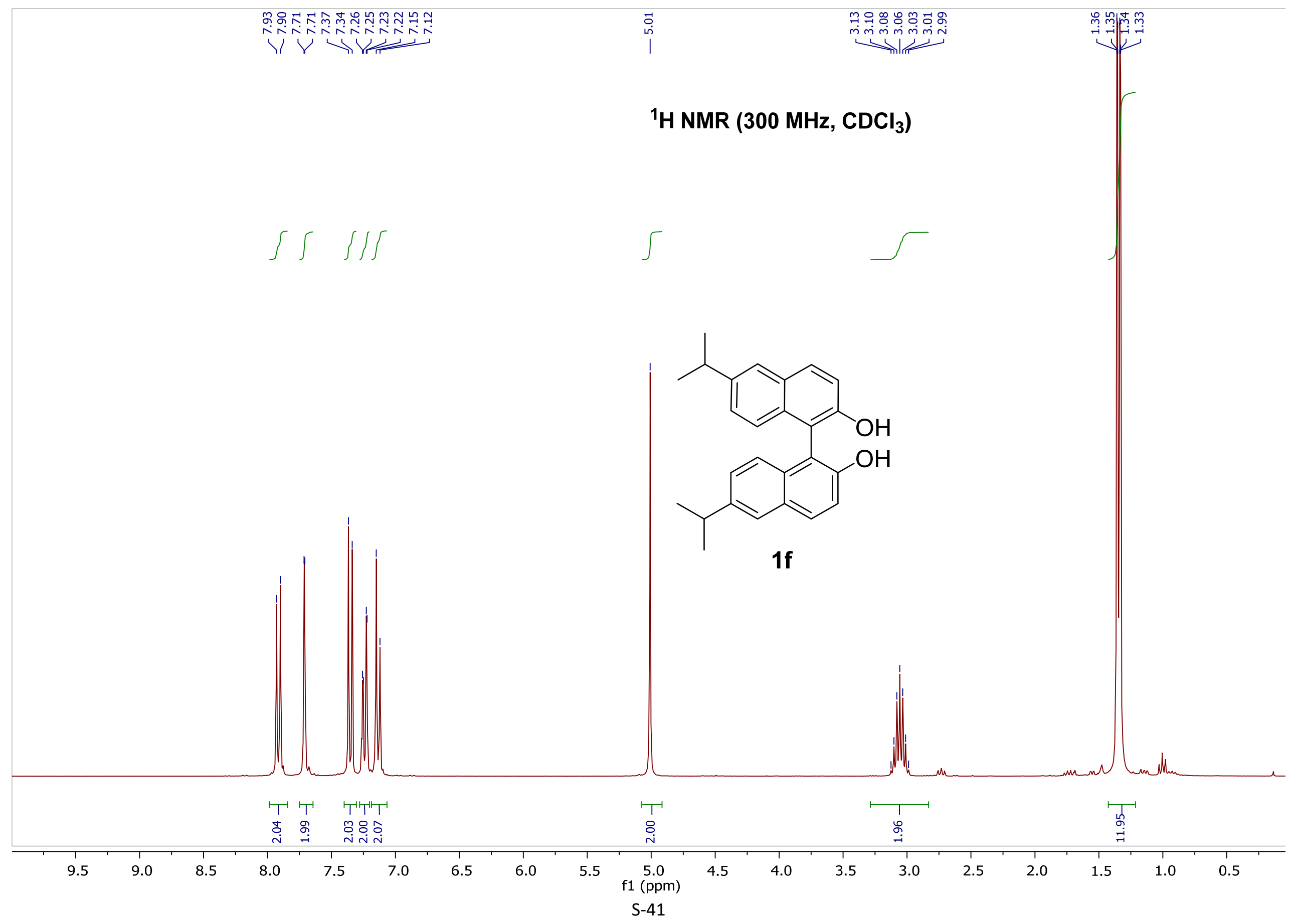




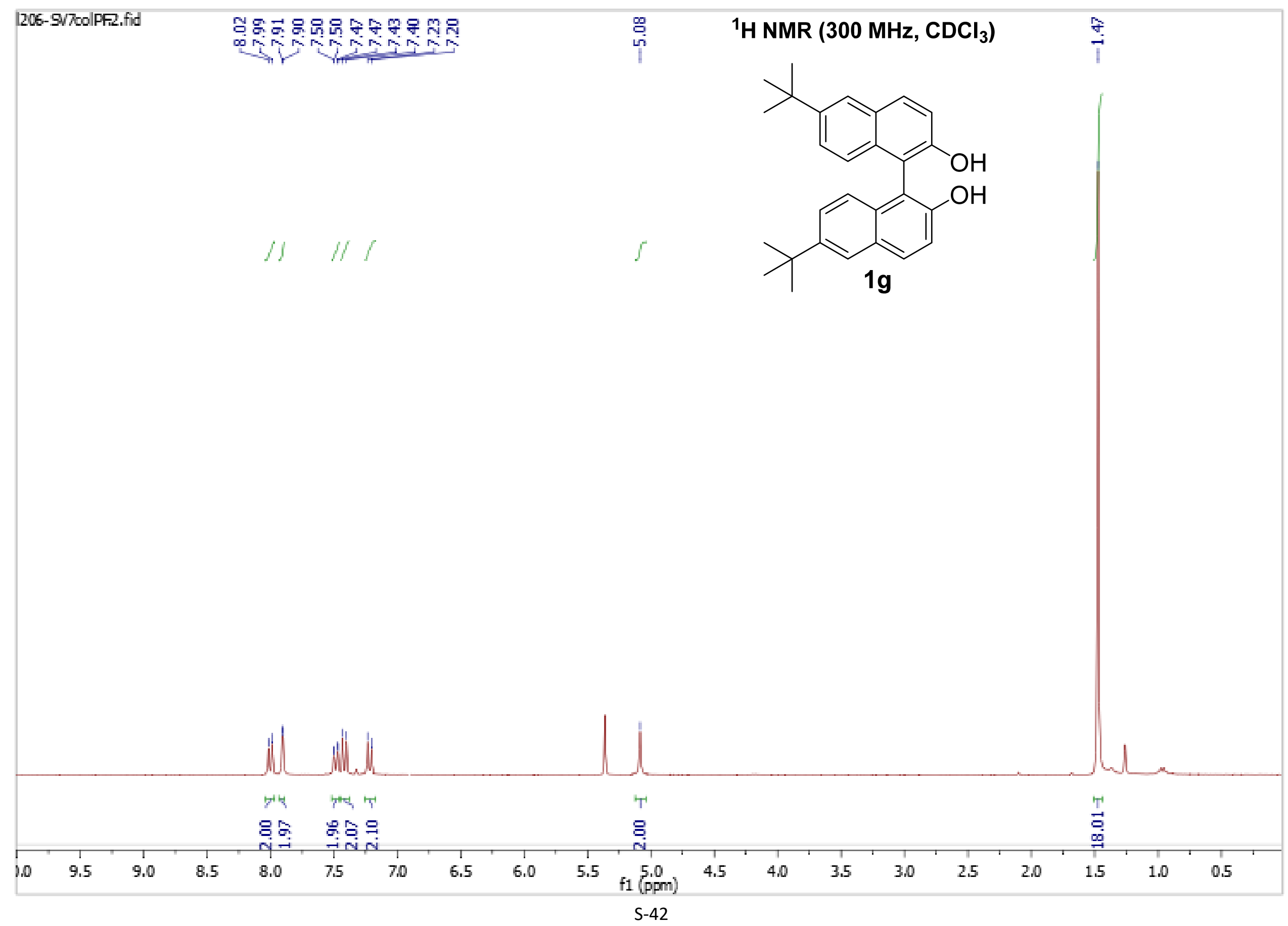




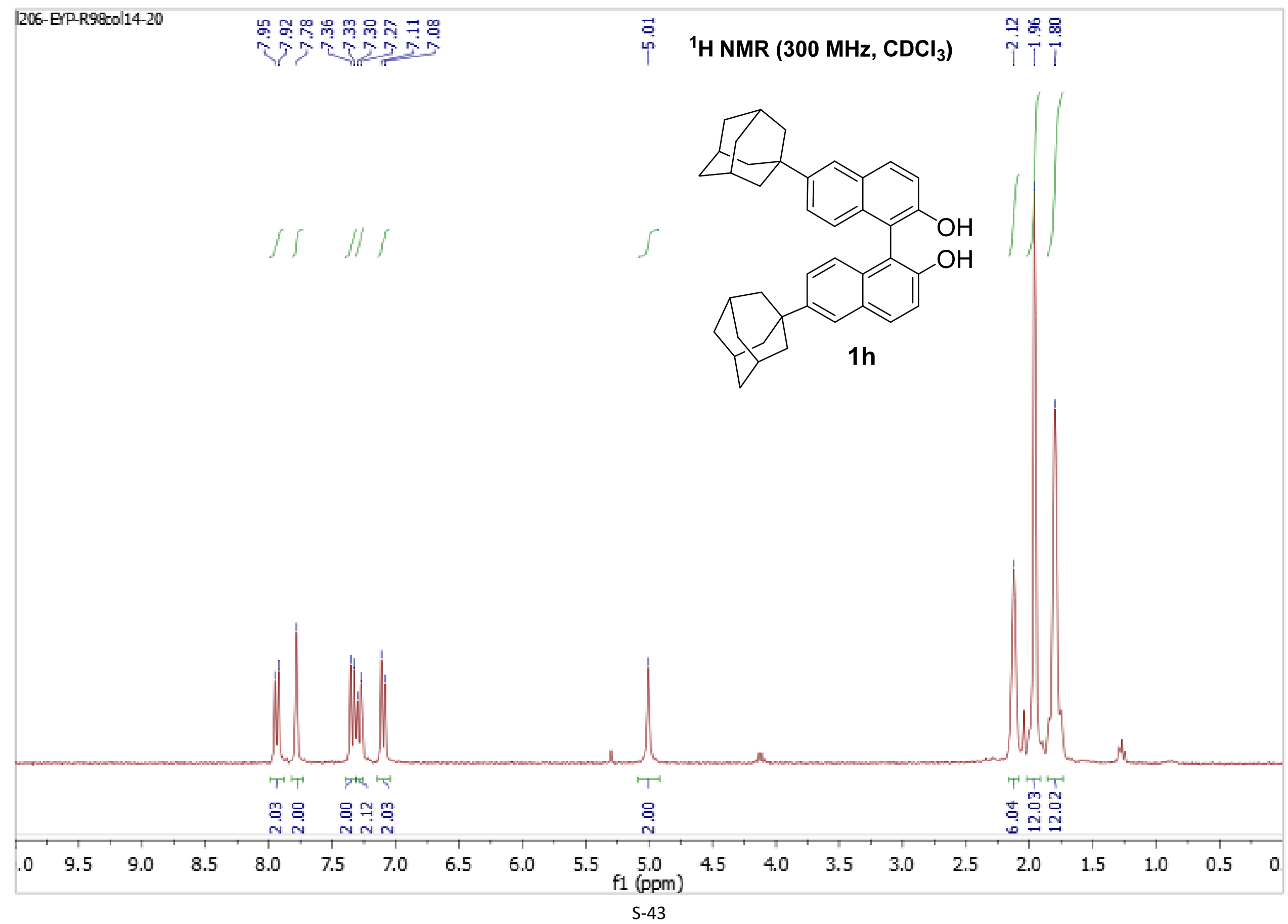




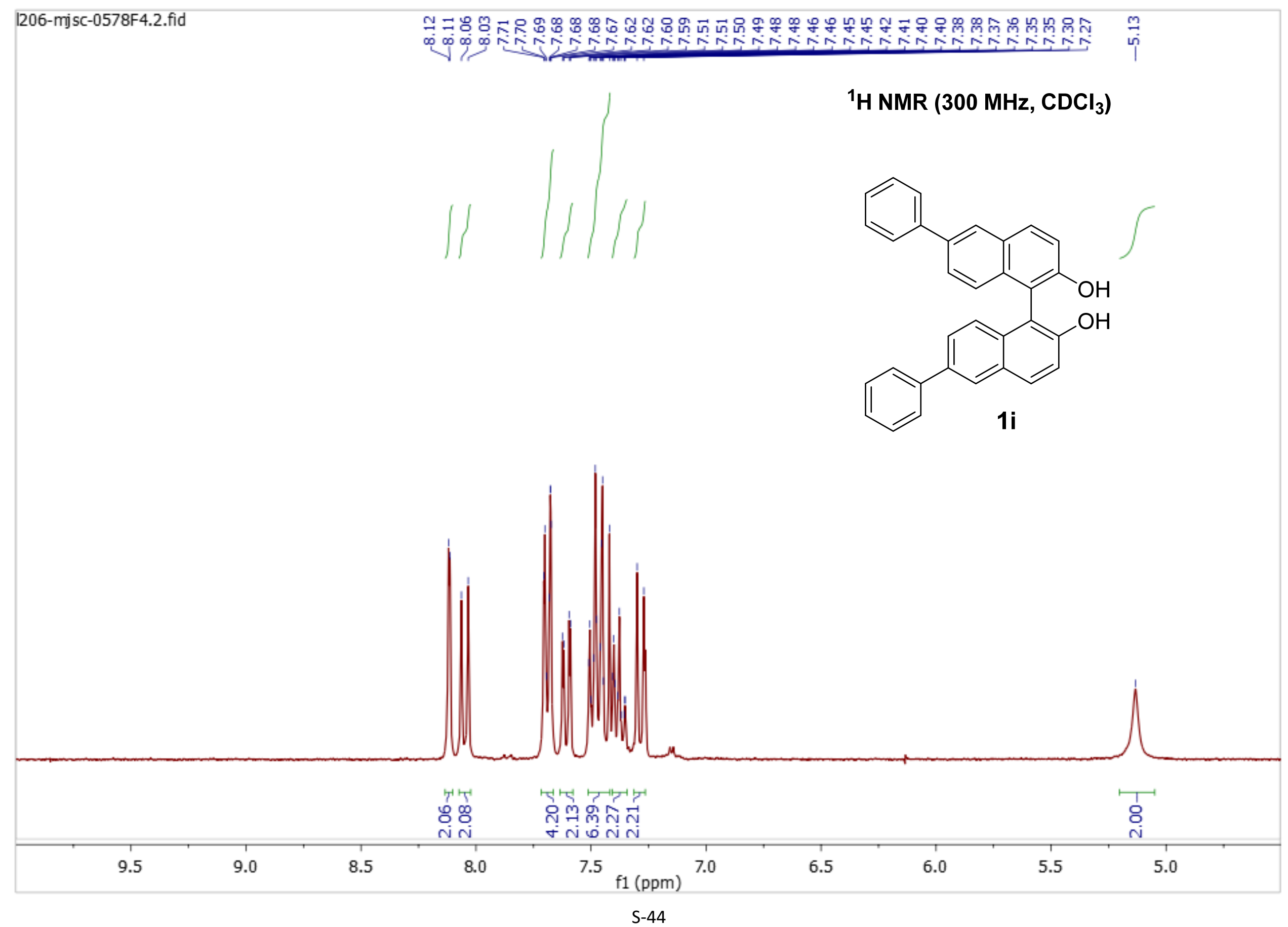




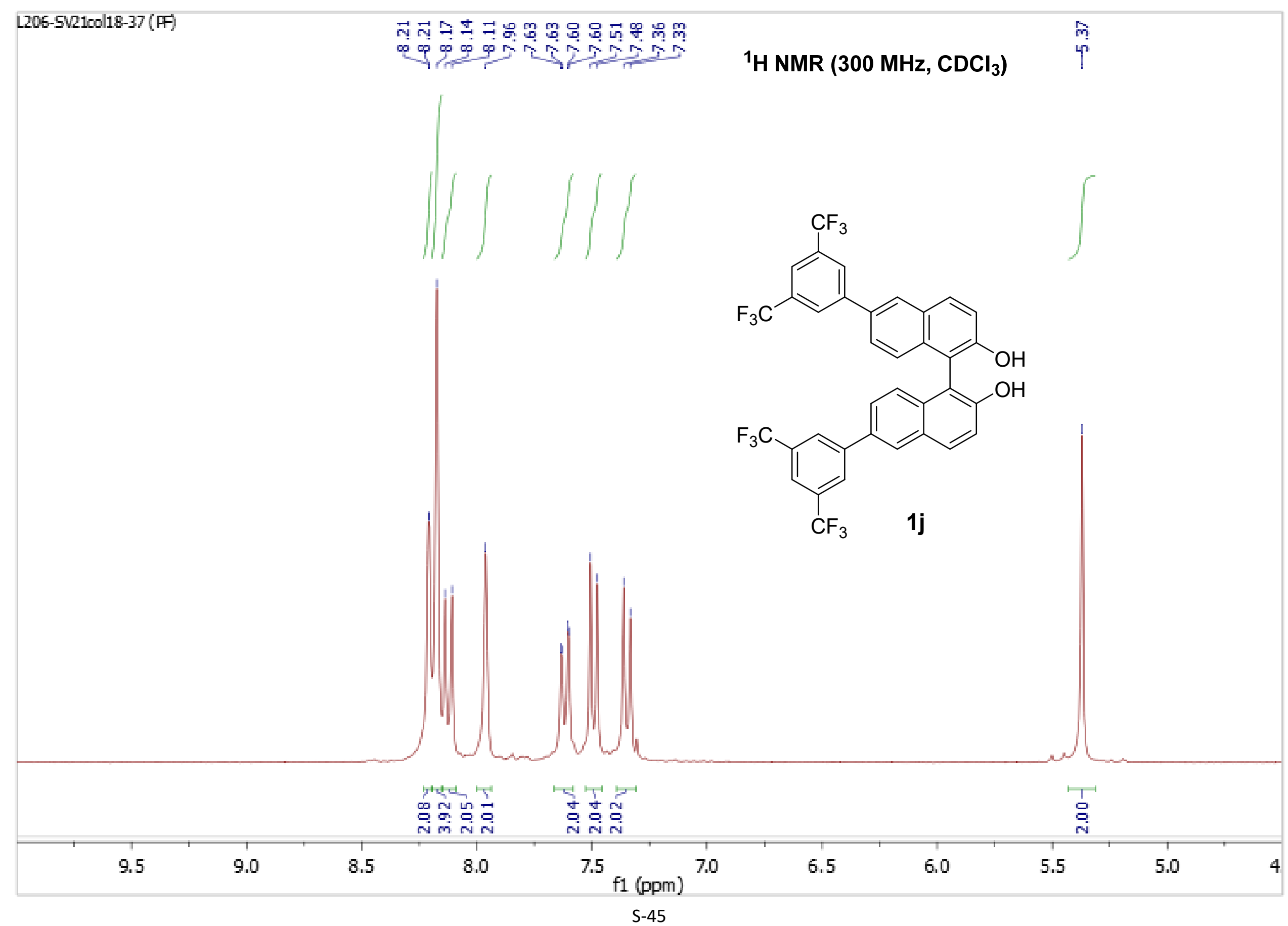




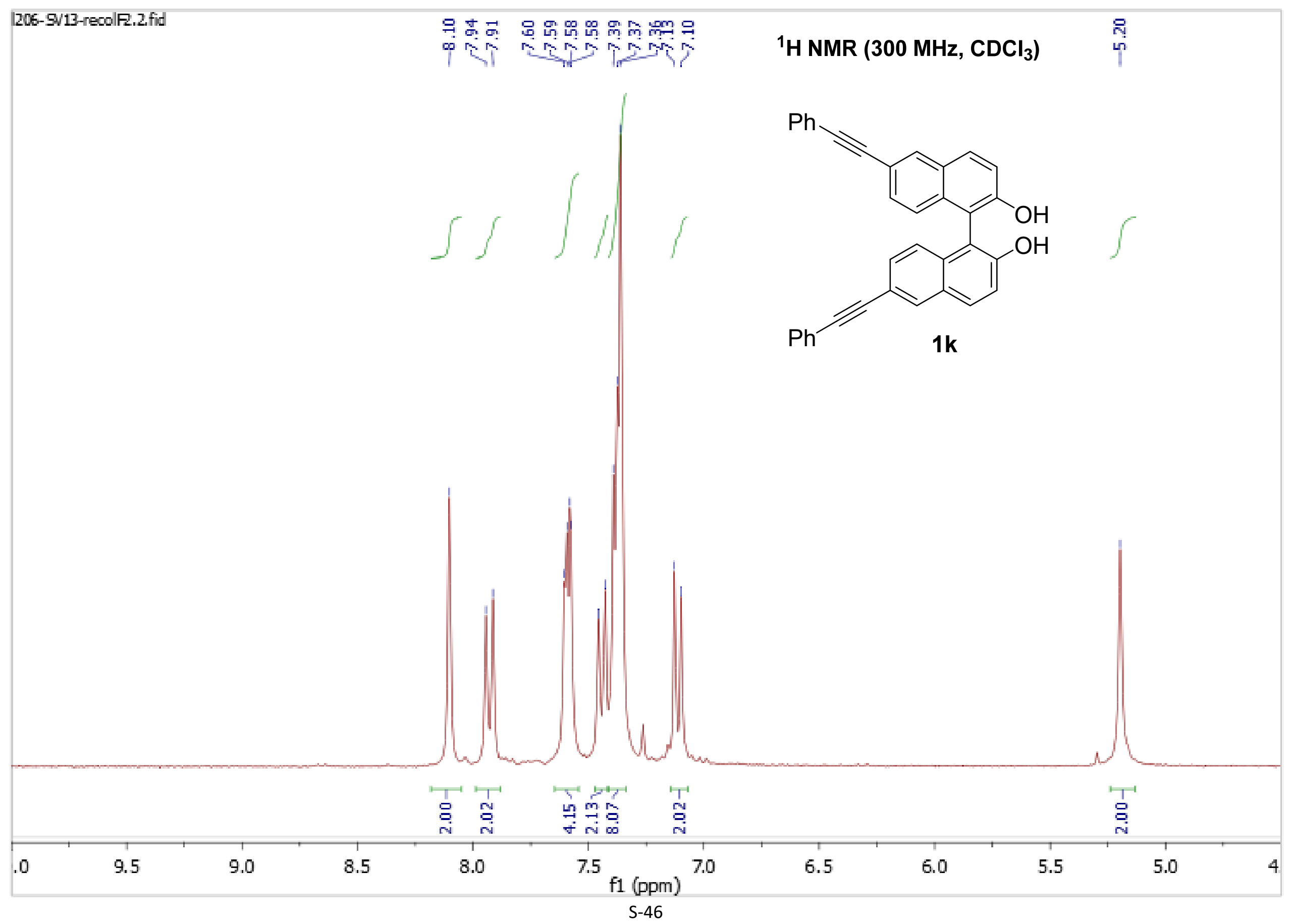




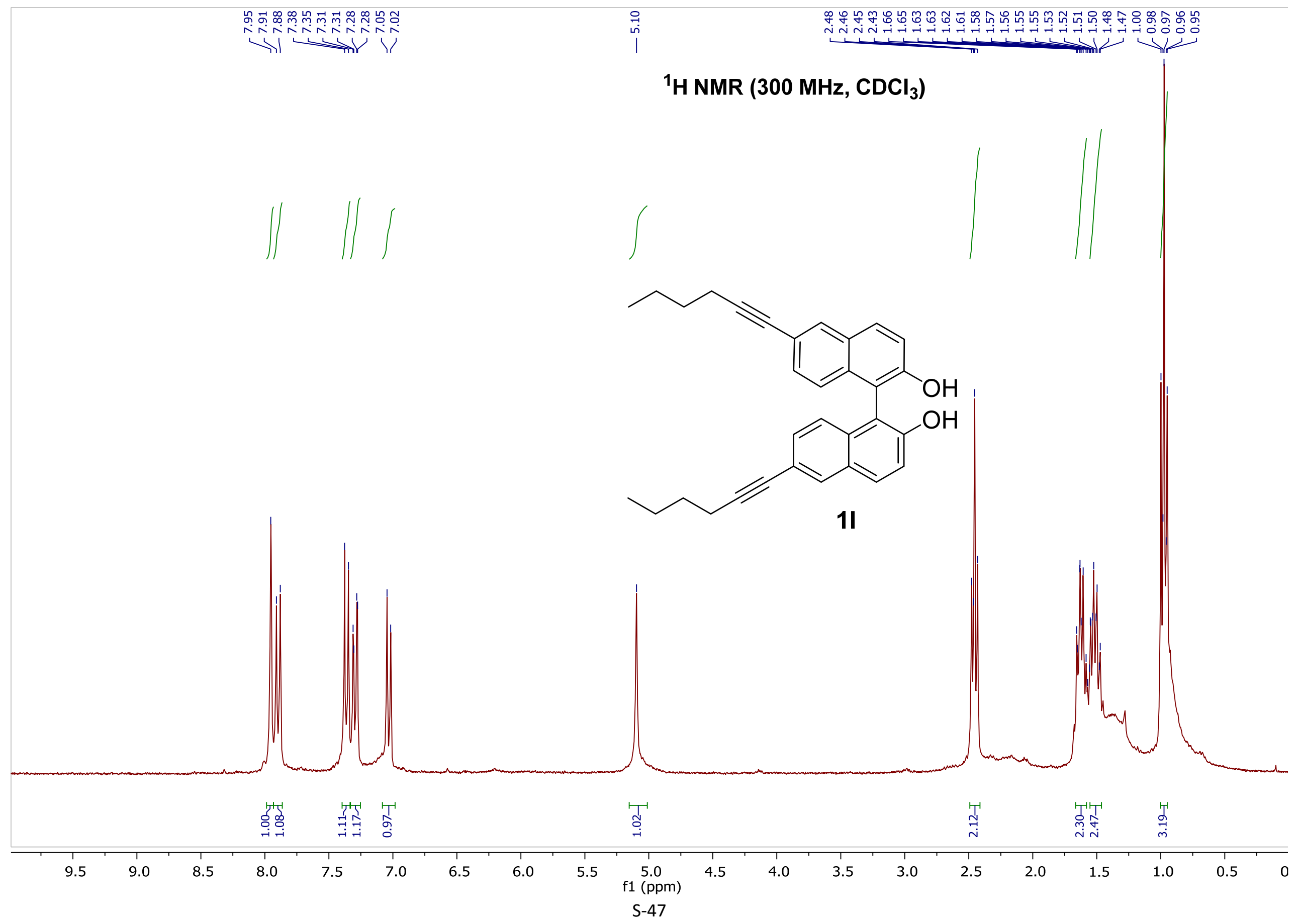




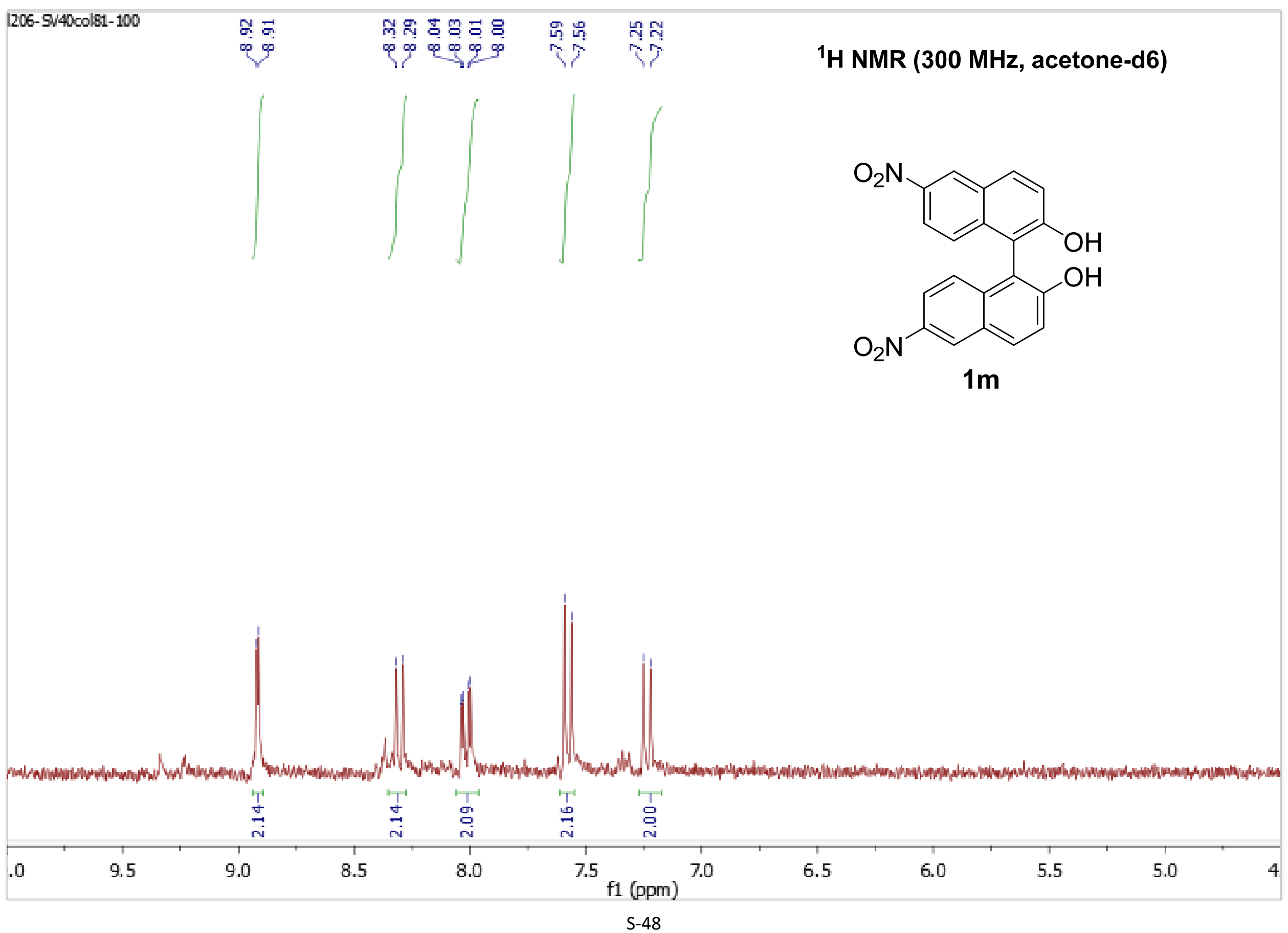




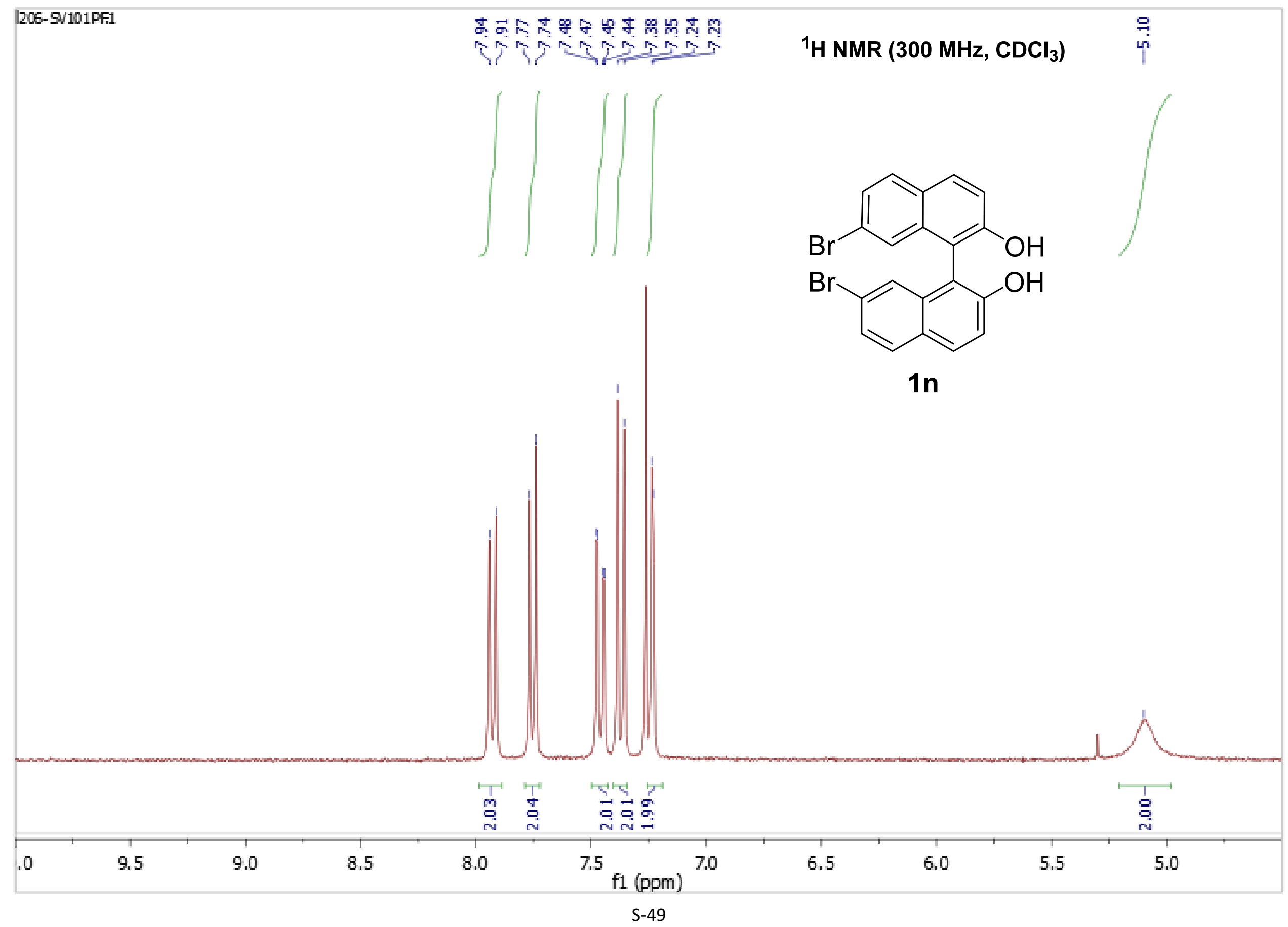




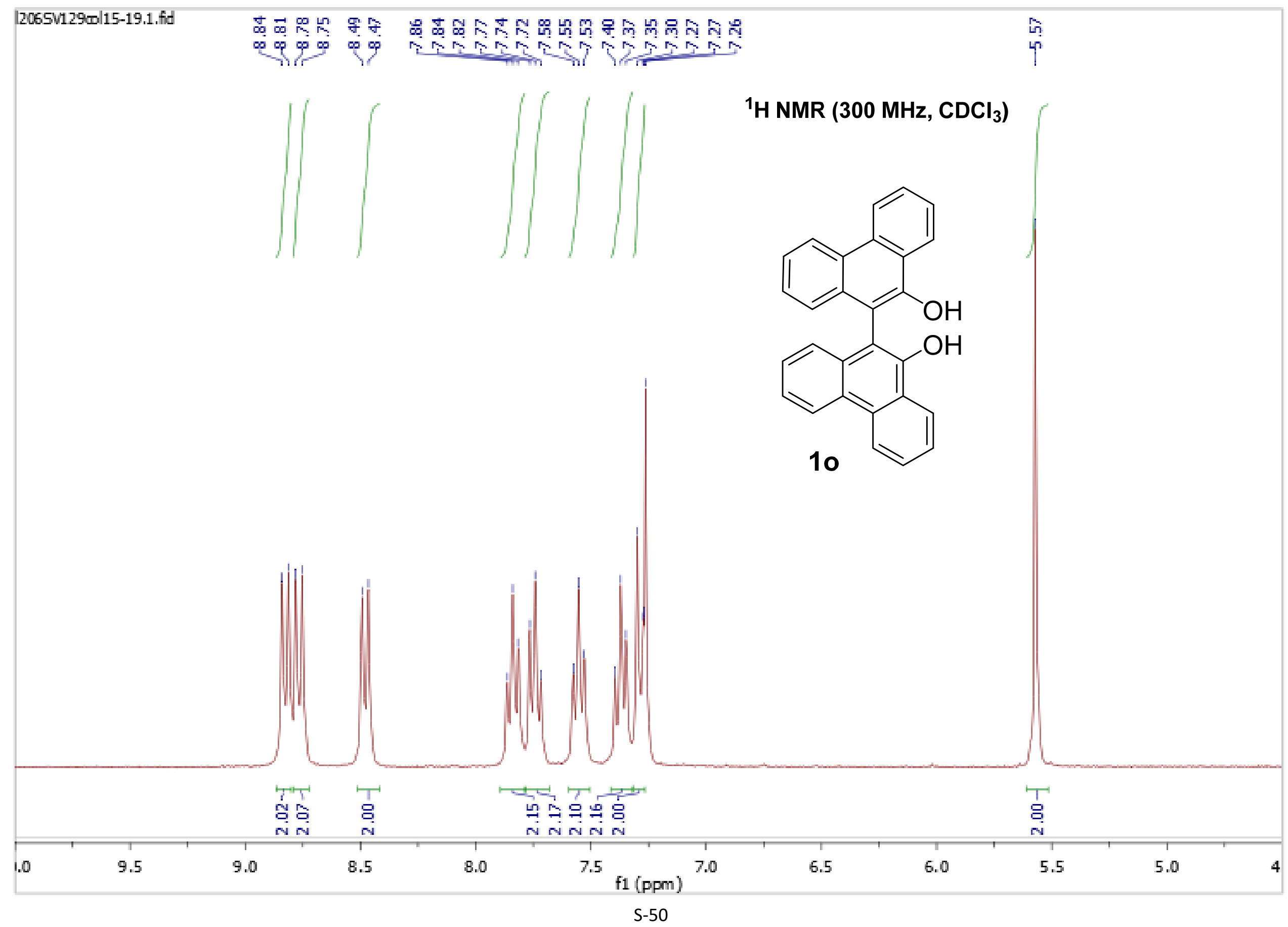




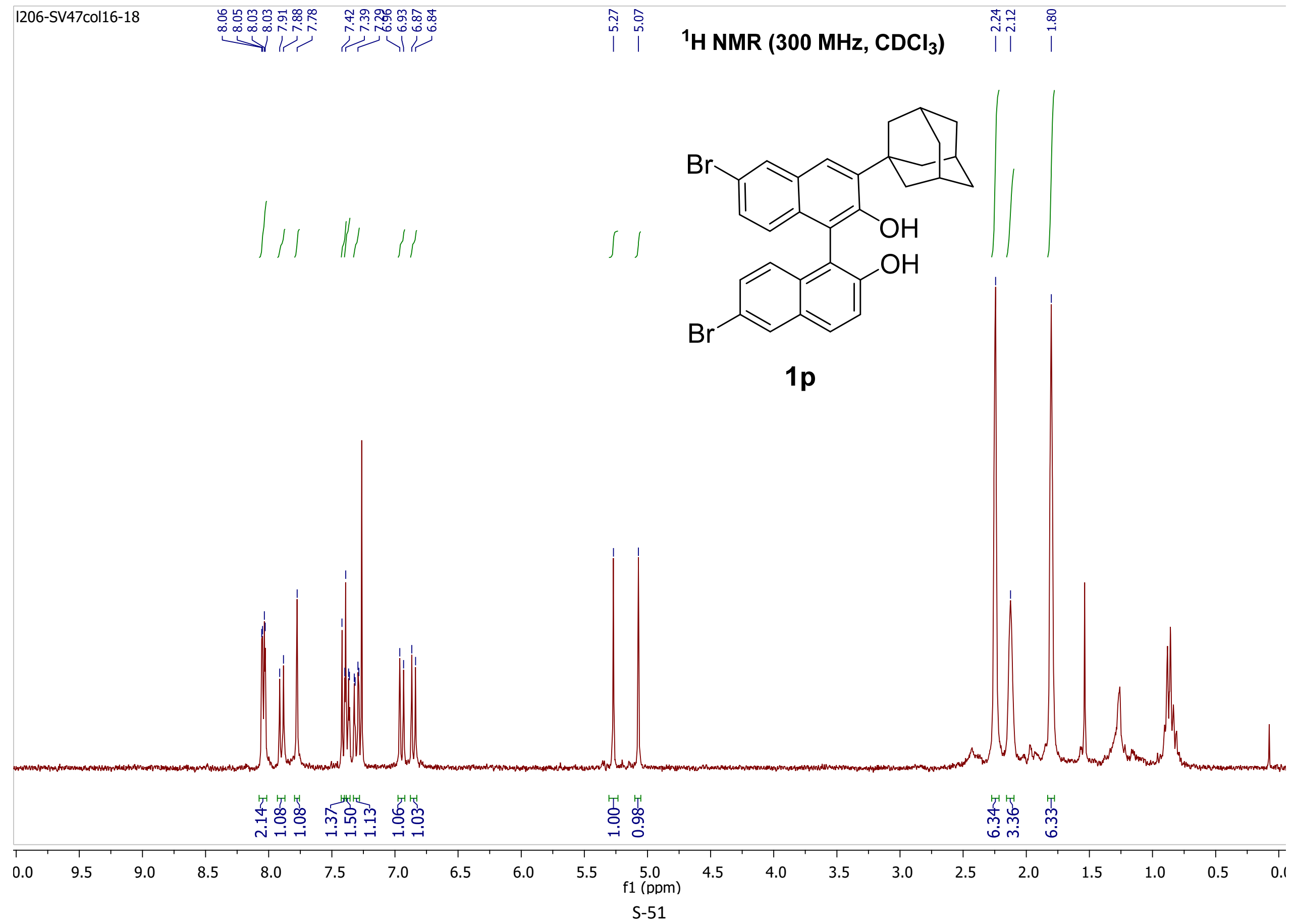




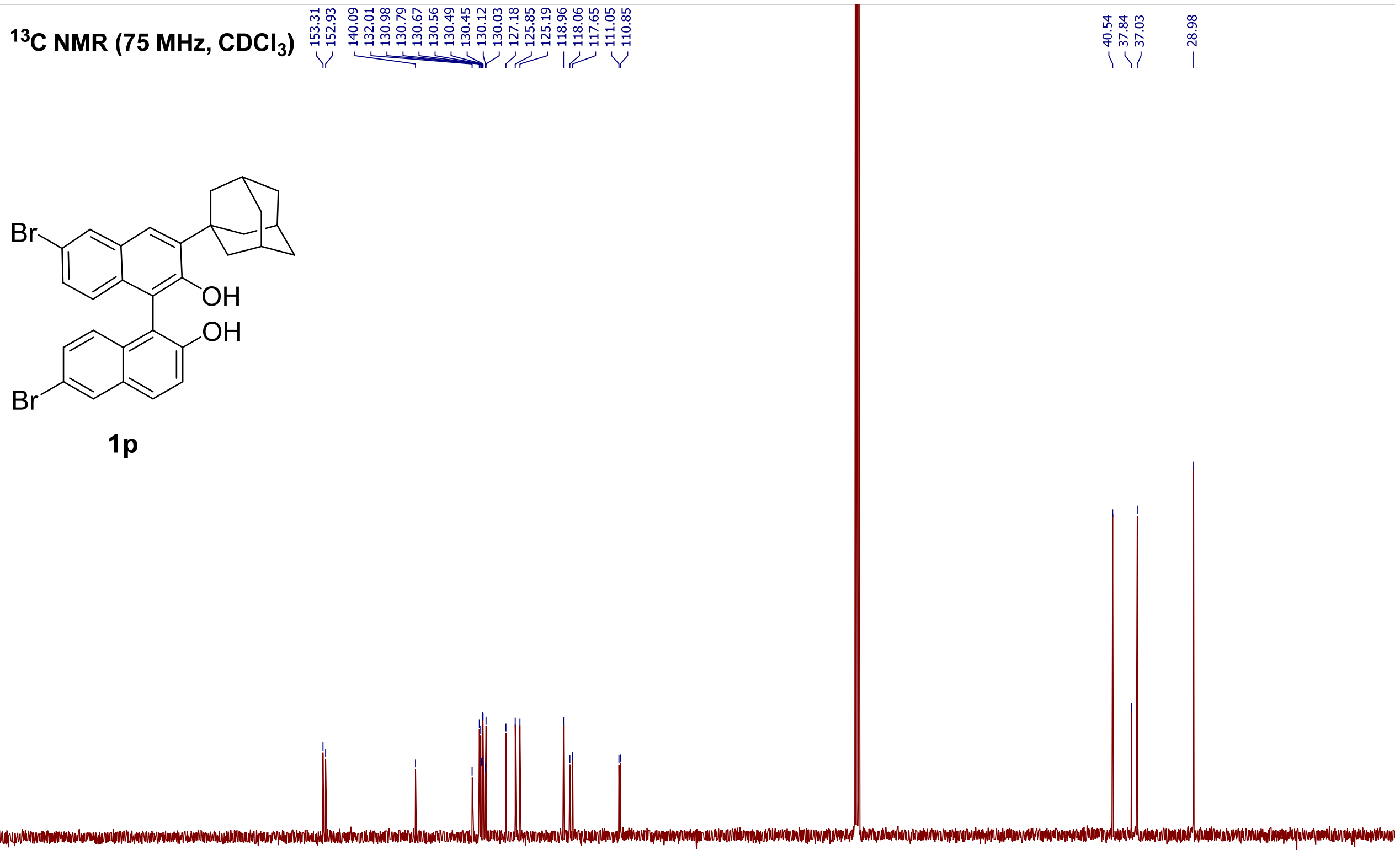

\begin{tabular}{|c|c|c|c|c|c|c|c|c|c|c|c|c|c|c|c|c|c|c|}
\hline $\begin{array}{l}1 \\
190\end{array}$ & $\begin{array}{l} \\
180\end{array}$ & 170 & 160 & $\begin{array}{l}1 \\
150\end{array}$ & 140 & 130 & 120 & 110 & $\begin{array}{c}100 \\
\mathrm{f} 1(\mathrm{ppm})\end{array}$ & 90 & 80 & 70 & 60 & 50 & 40 & 30 & 20 & 10 \\
\hline
\end{tabular}




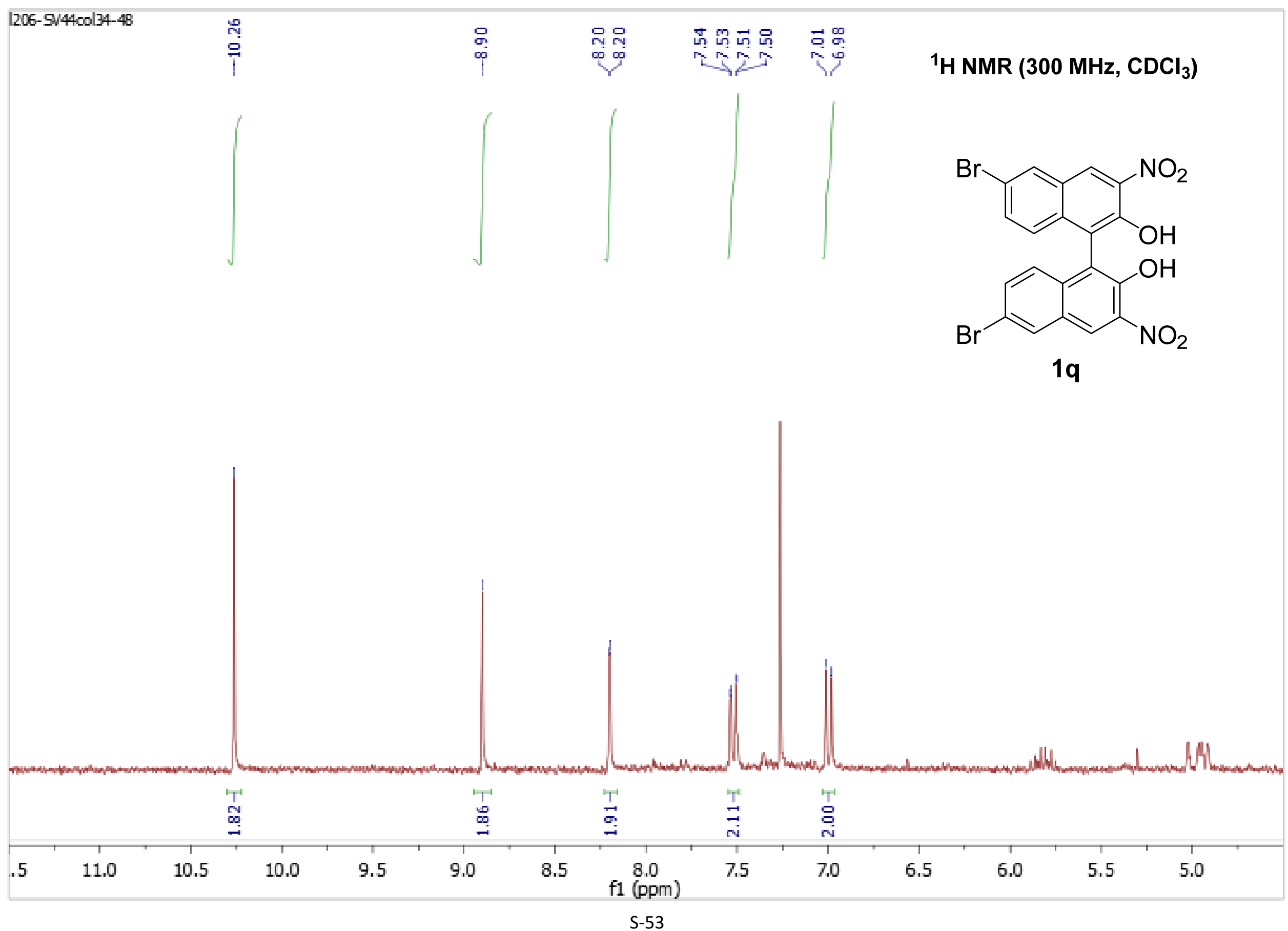




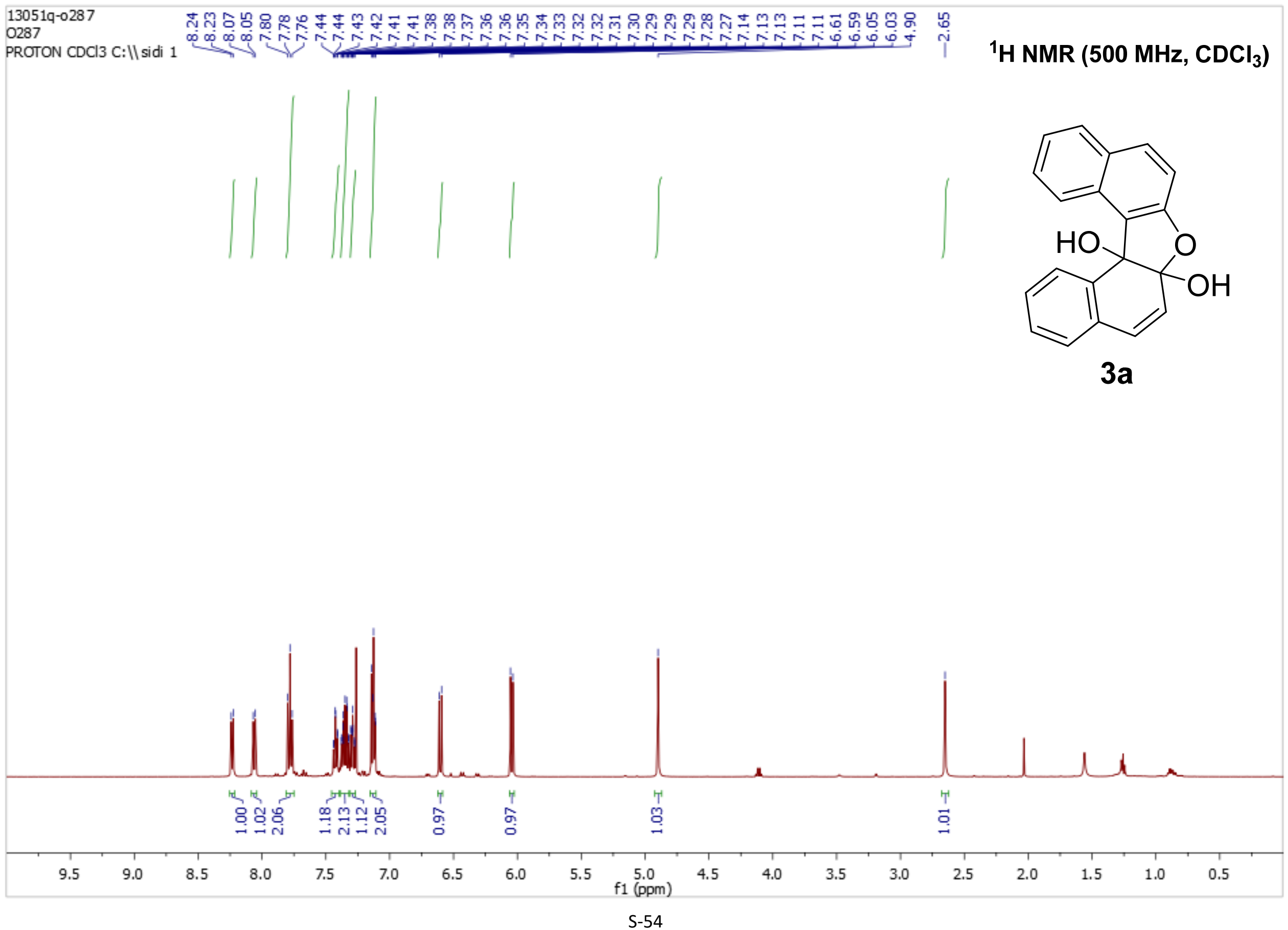




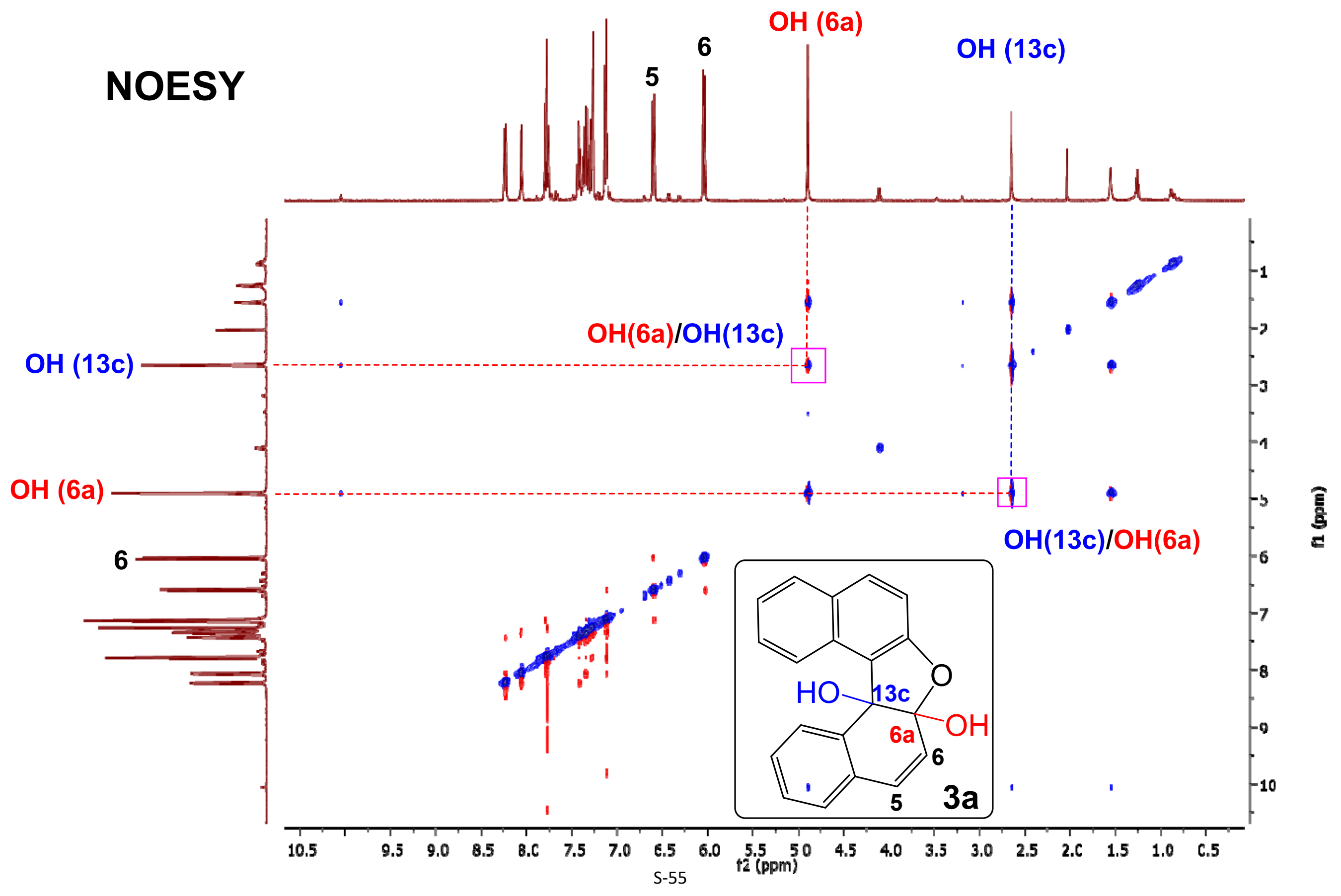




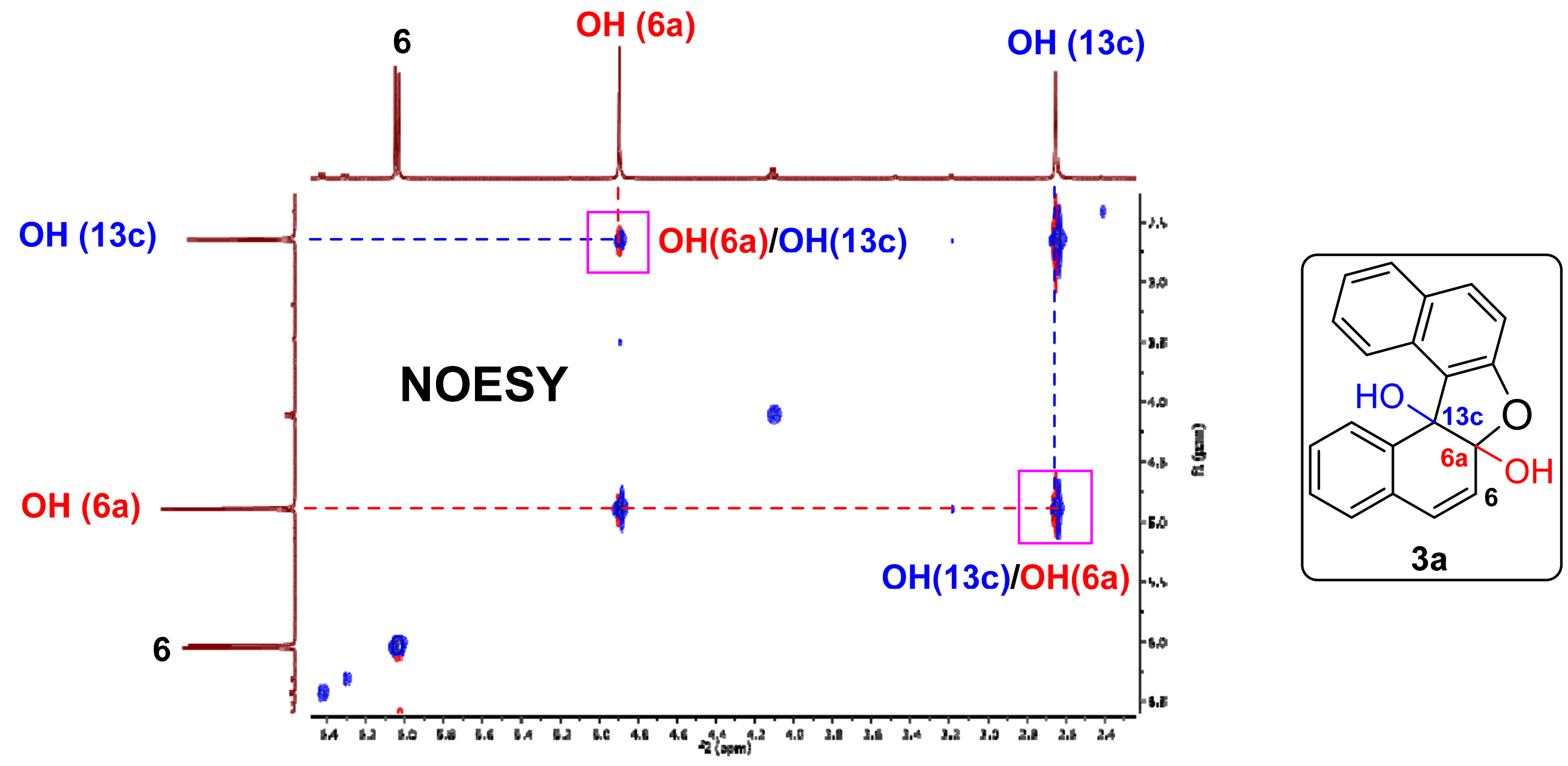


${ }^{13} \mathrm{C}$ NMR (75 MHz, $\mathrm{CDCl}_{3}$ )

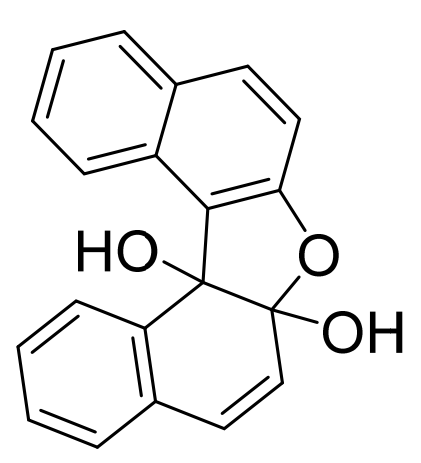

$3 a$

\section{$\underset{\substack{7 \\ i}}{\sim}$}

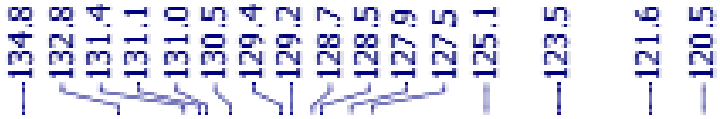

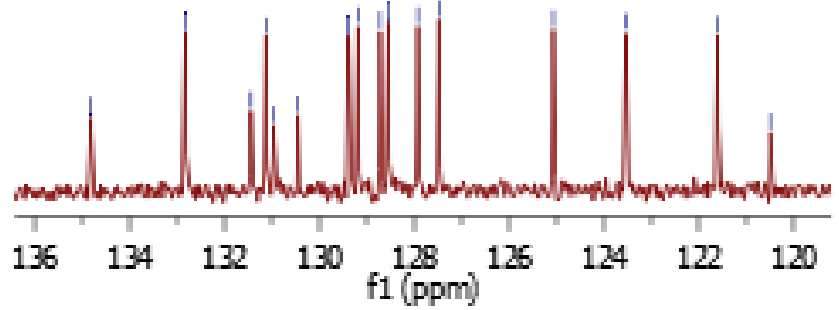

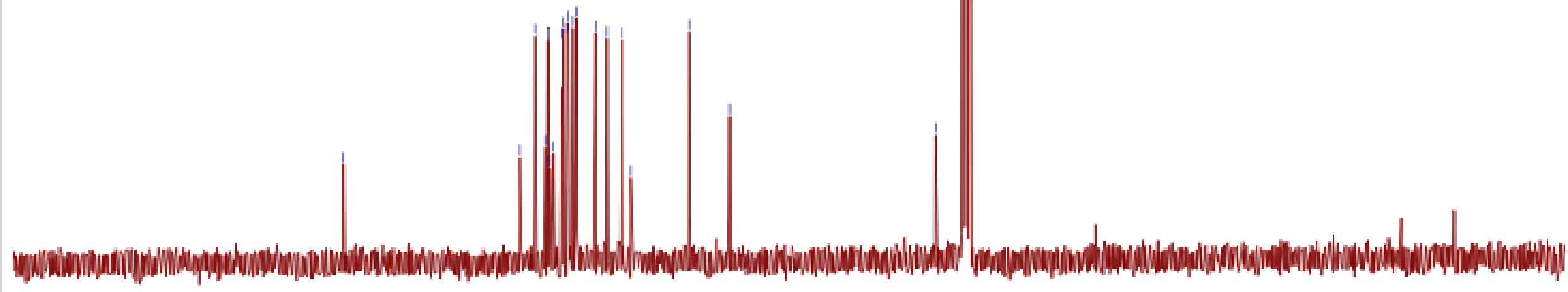

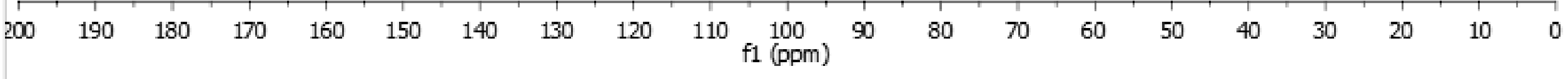




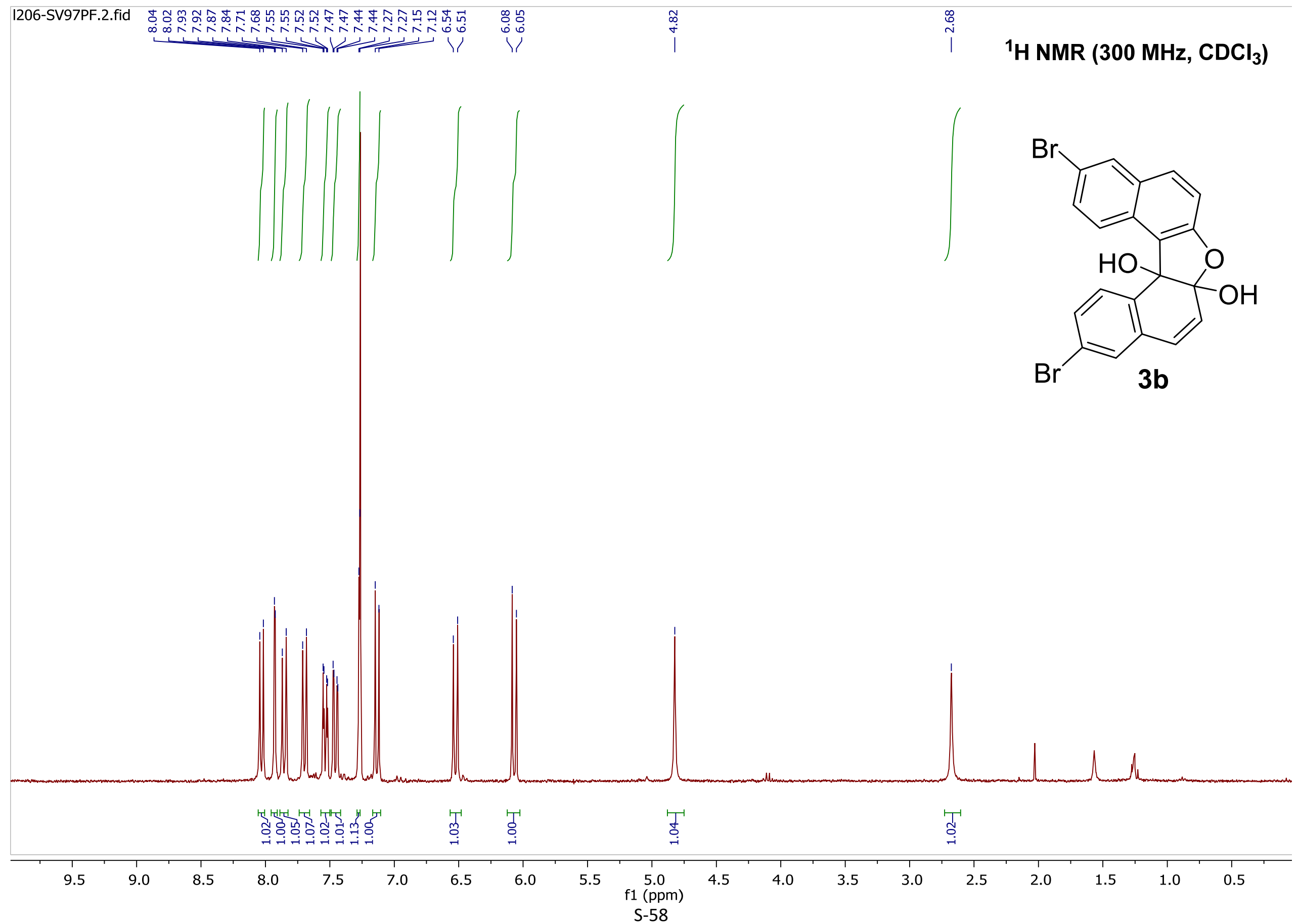


${ }^{13} \mathrm{C}$ NMR $\left(75 \mathrm{MHz}, \mathrm{CDCl}_{3}\right)$

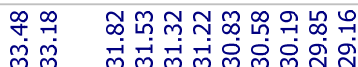

임

11 iोlitit?<smiles>OC12C=Cc3cc(Br)ccc3C1(O)c1c(ccc3cc(Br)ccc13)O2</smiles>

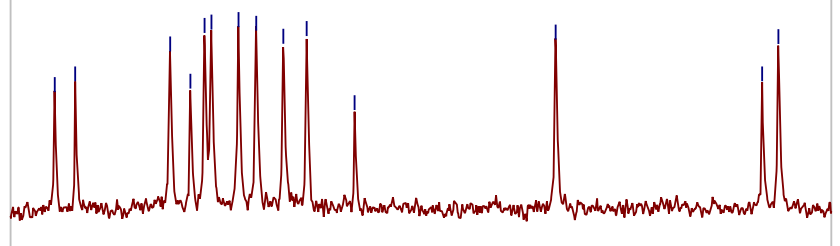

$\begin{array}{lllllllllllll}134 & 133 & 132 & 131 & 130 & 129 & 128 & 122 & 126 & 125 & 124 & 123\end{array}$ $1(\mathrm{ppm})$

$\mathrm{Br} \quad 3 \mathbf{b}$

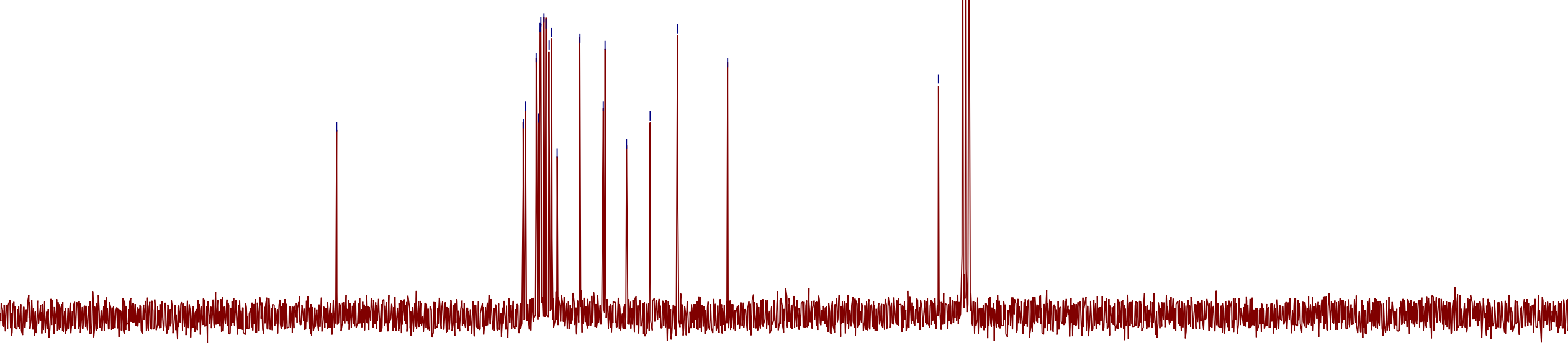




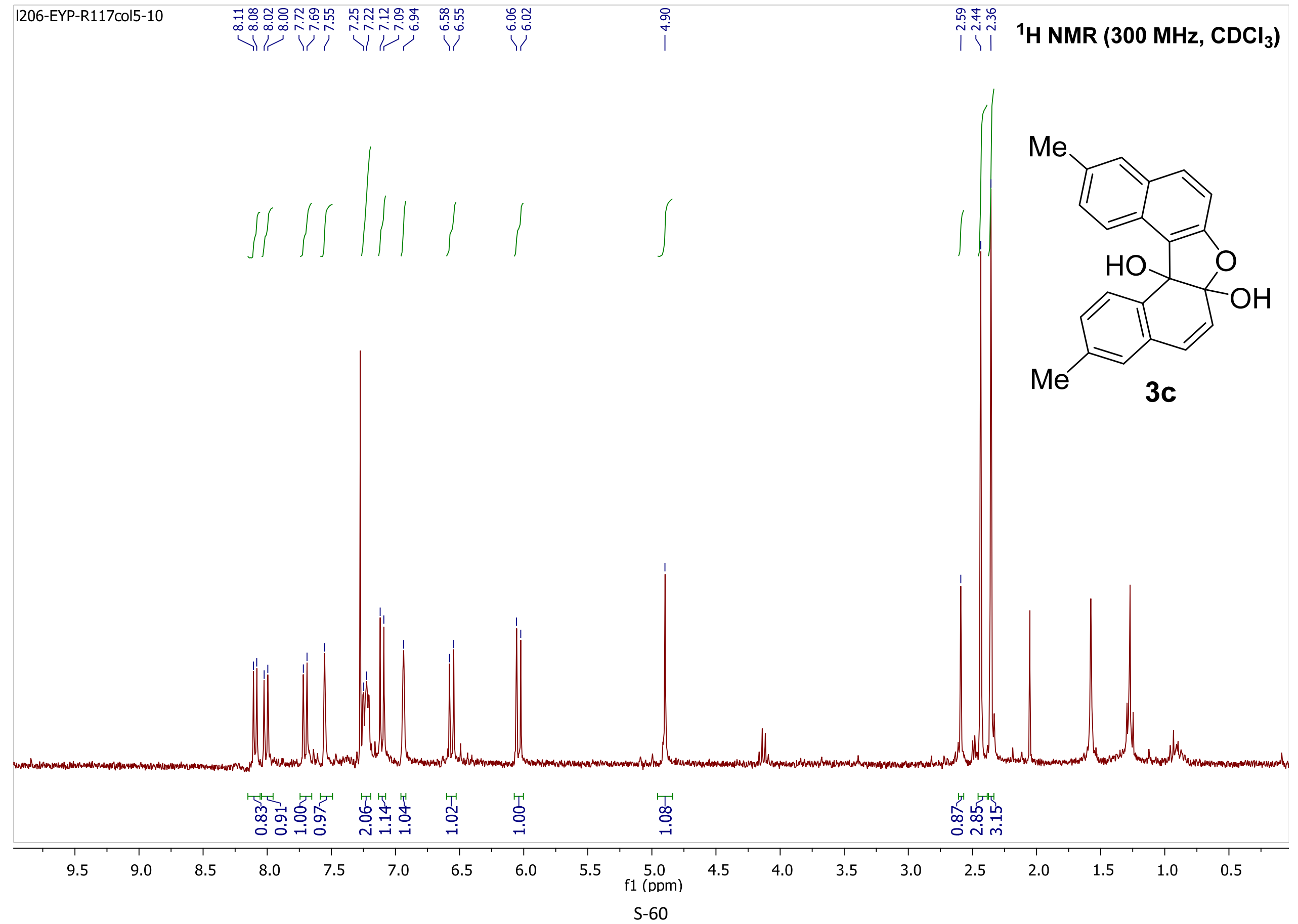




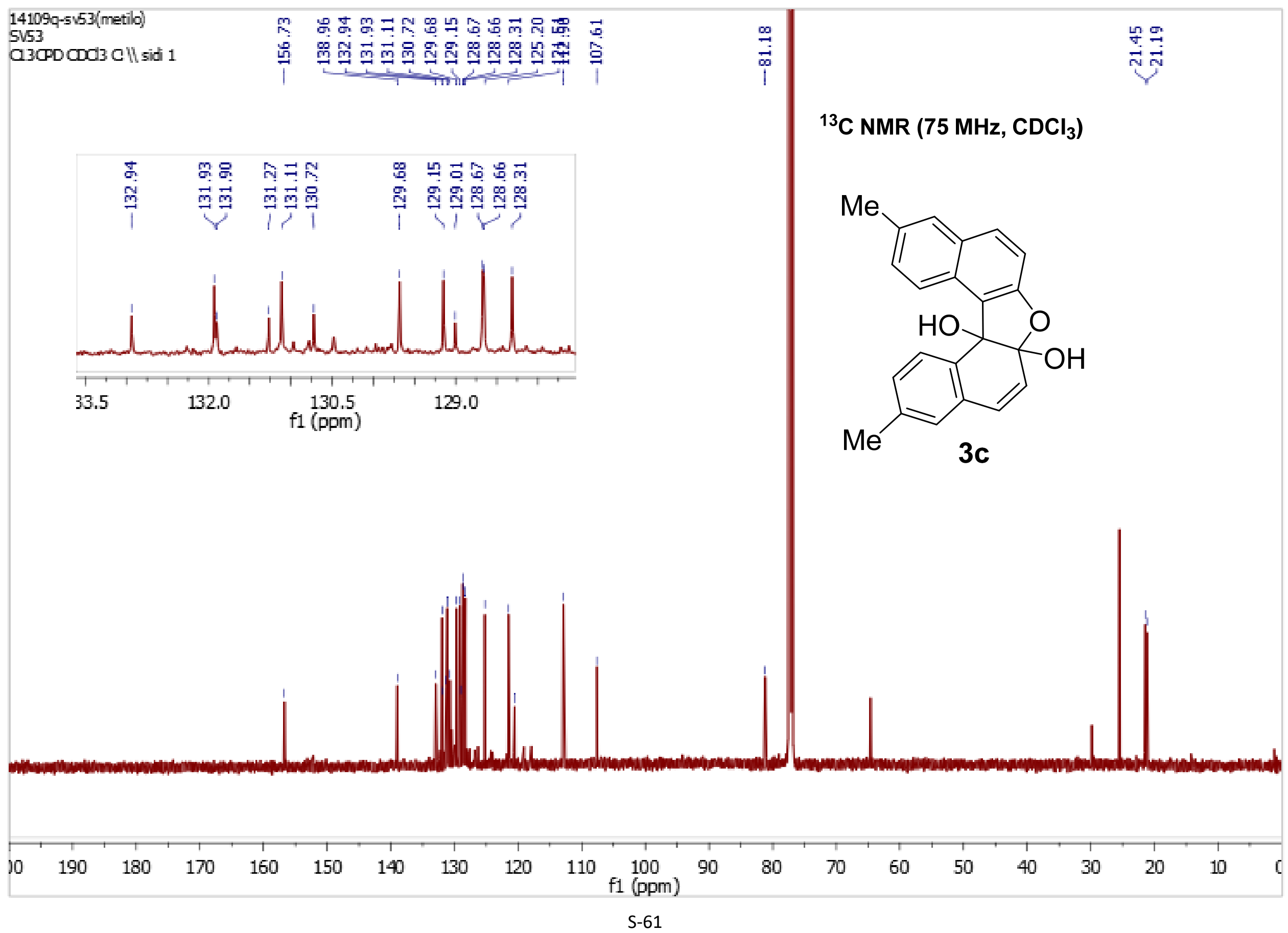




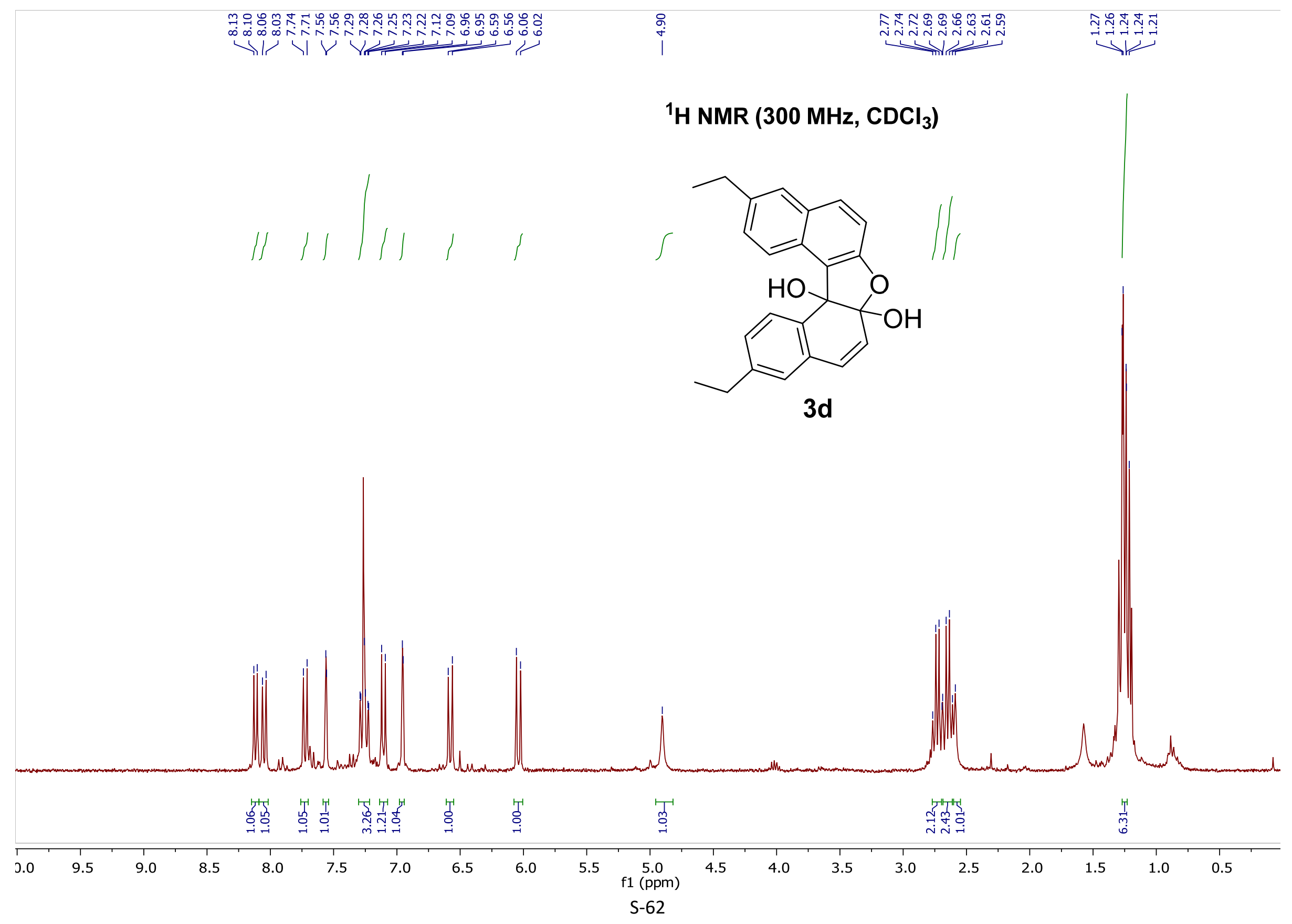




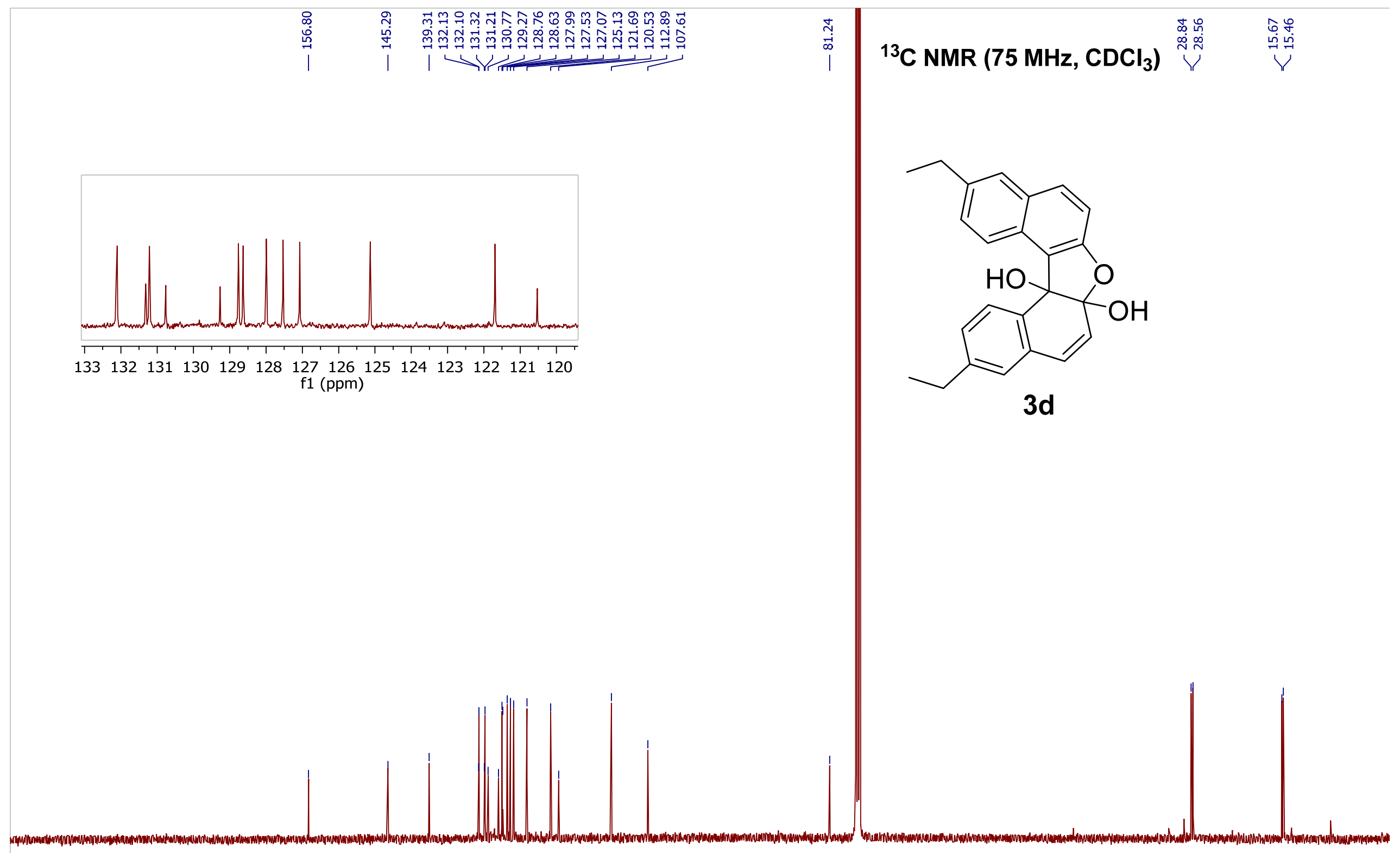

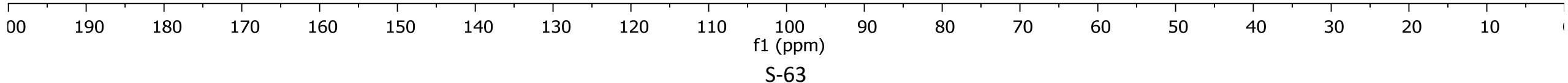




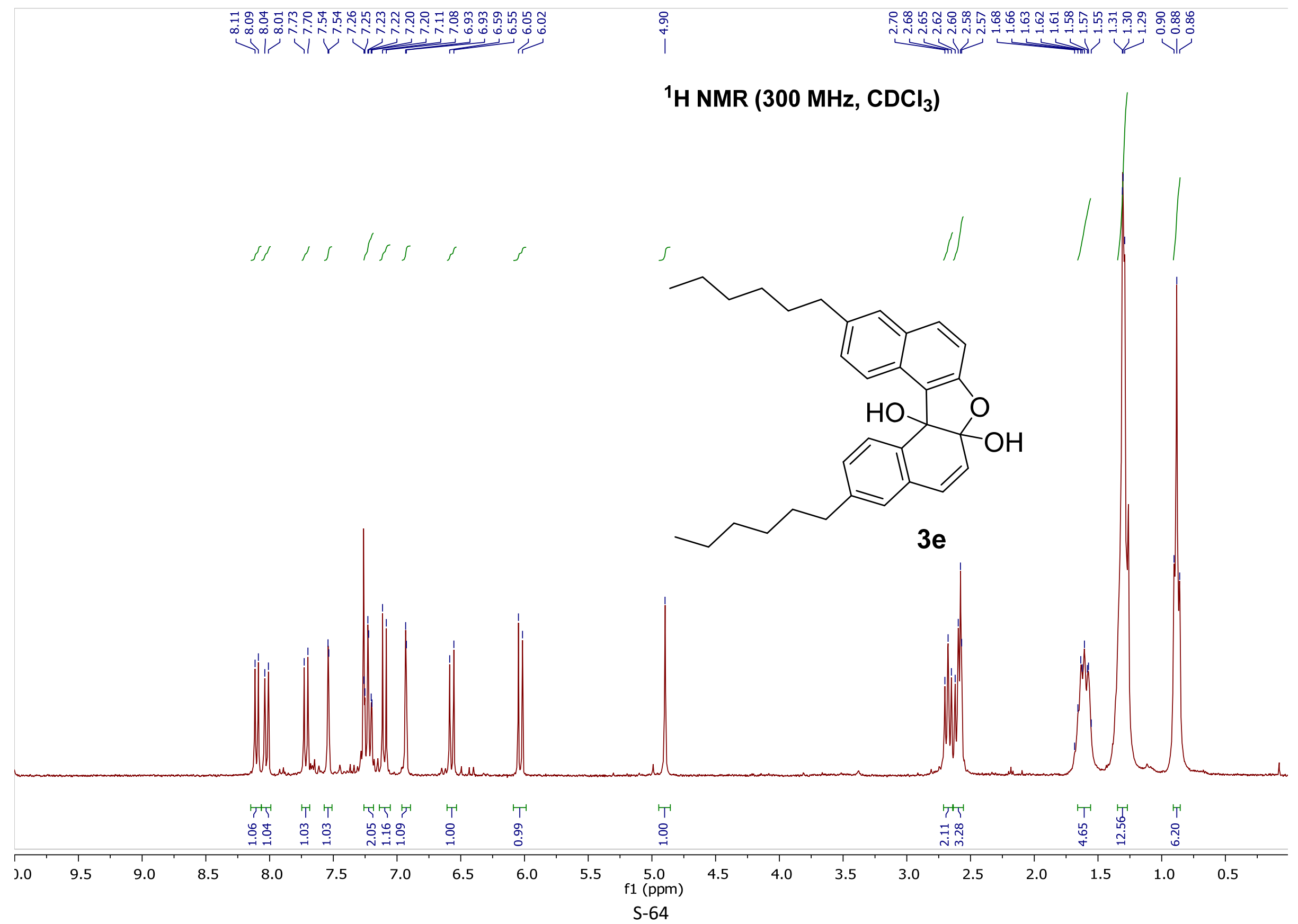




\section{$\begin{array}{lll}0 & \infty & 0\end{array}$ \\ 1}

${ }^{13} \mathrm{C}$ NMR (75 MHz, $\mathrm{CDCl}_{3}$ )

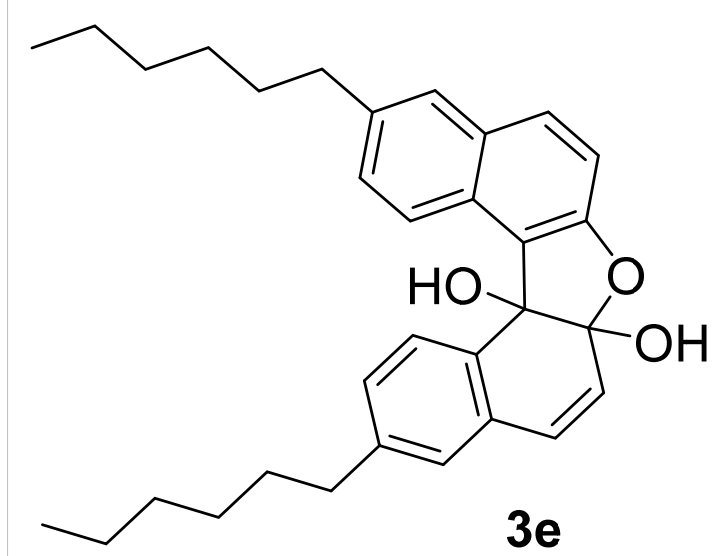

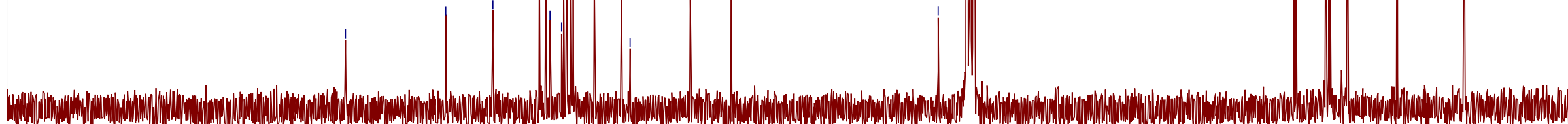

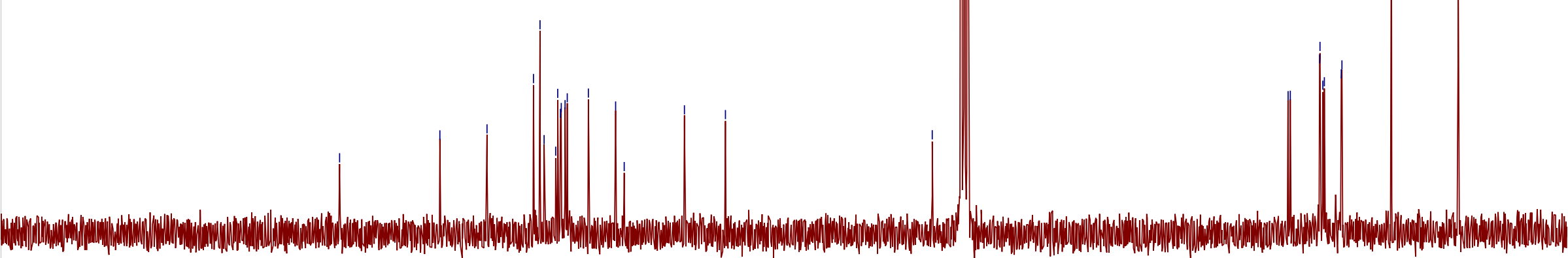




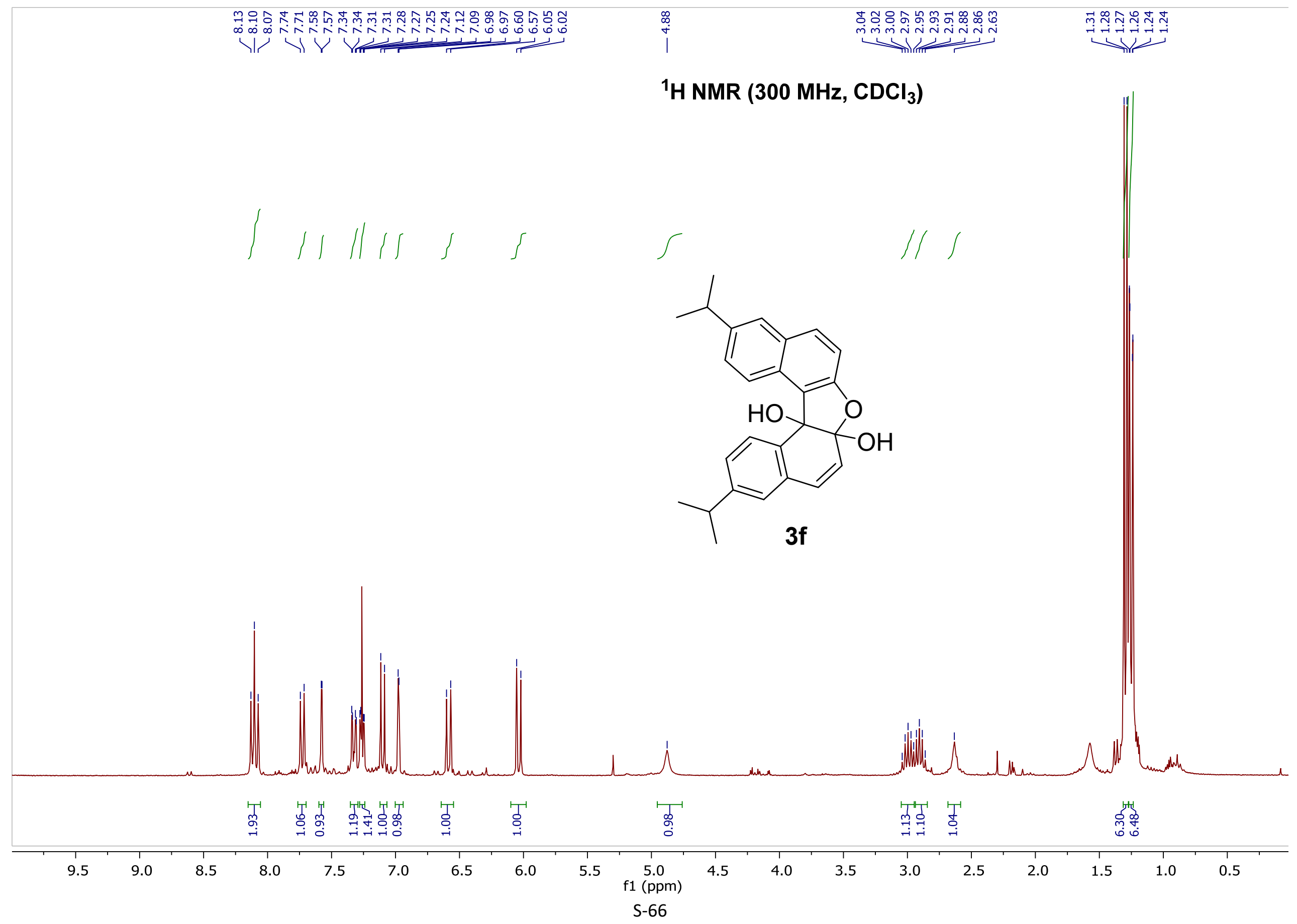




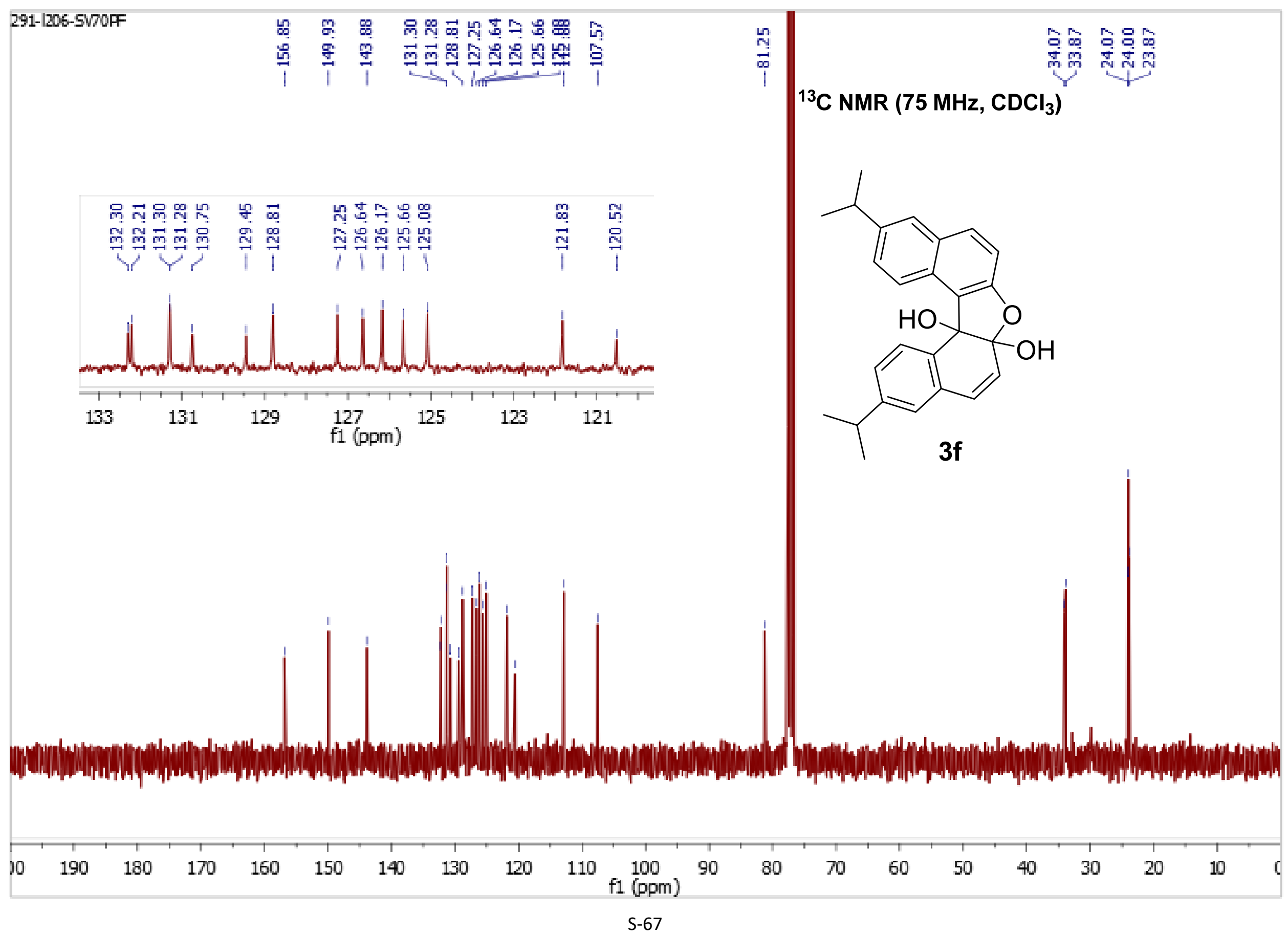




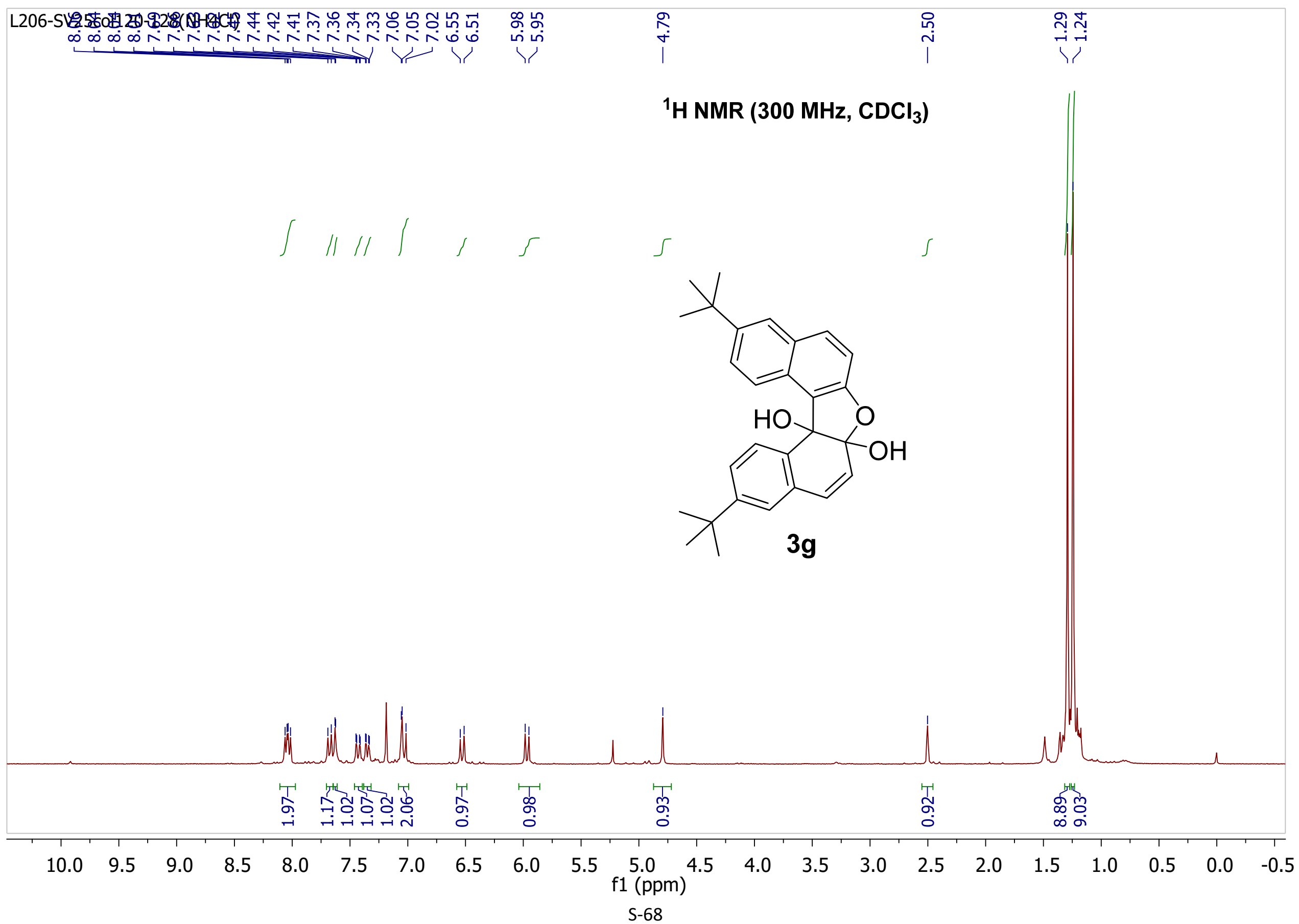




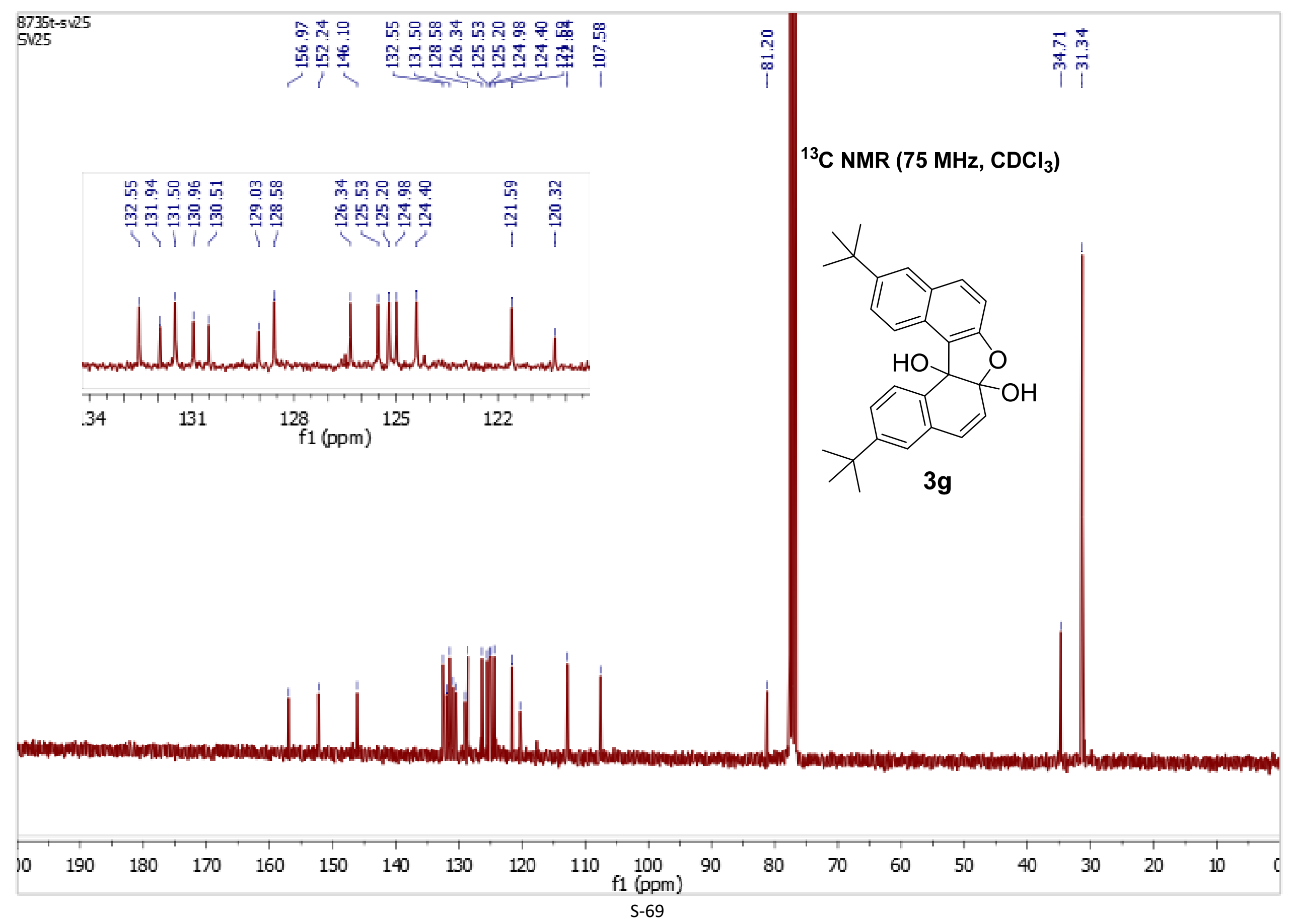




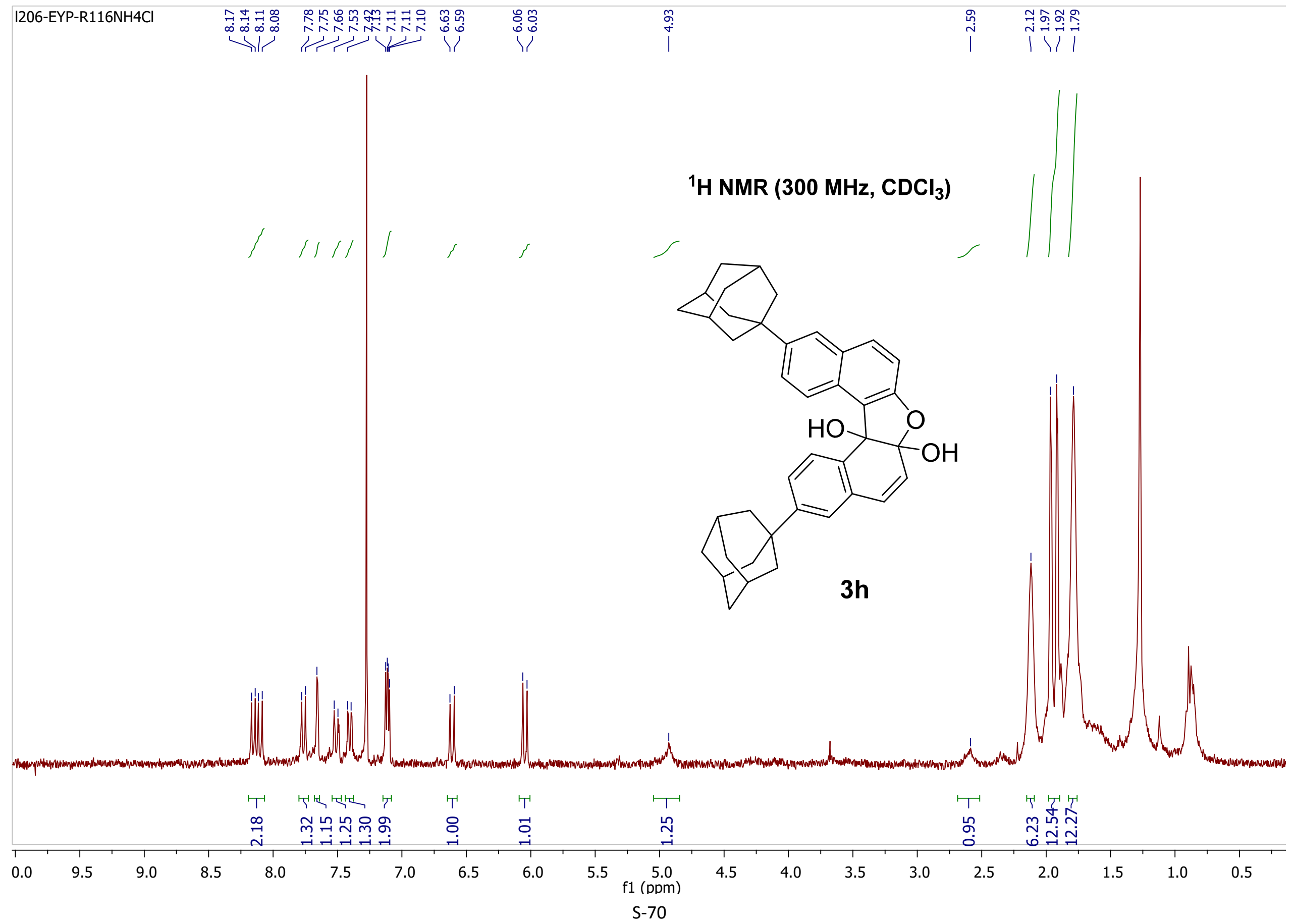


${ }^{13} \mathrm{C}$ NMR $\left(75 \mathrm{MHz}, \mathrm{CDCl}_{3}\right)$

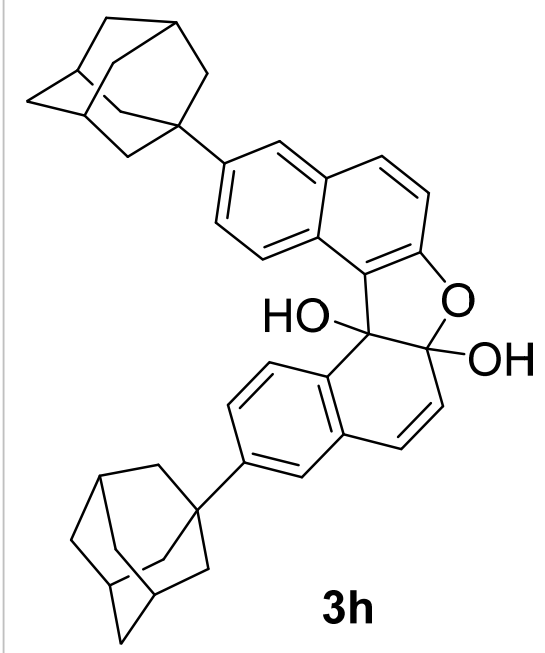

$00 \quad 190 \quad 180$

170

150

$140 \quad 130$

120

110

f1 (ppm)




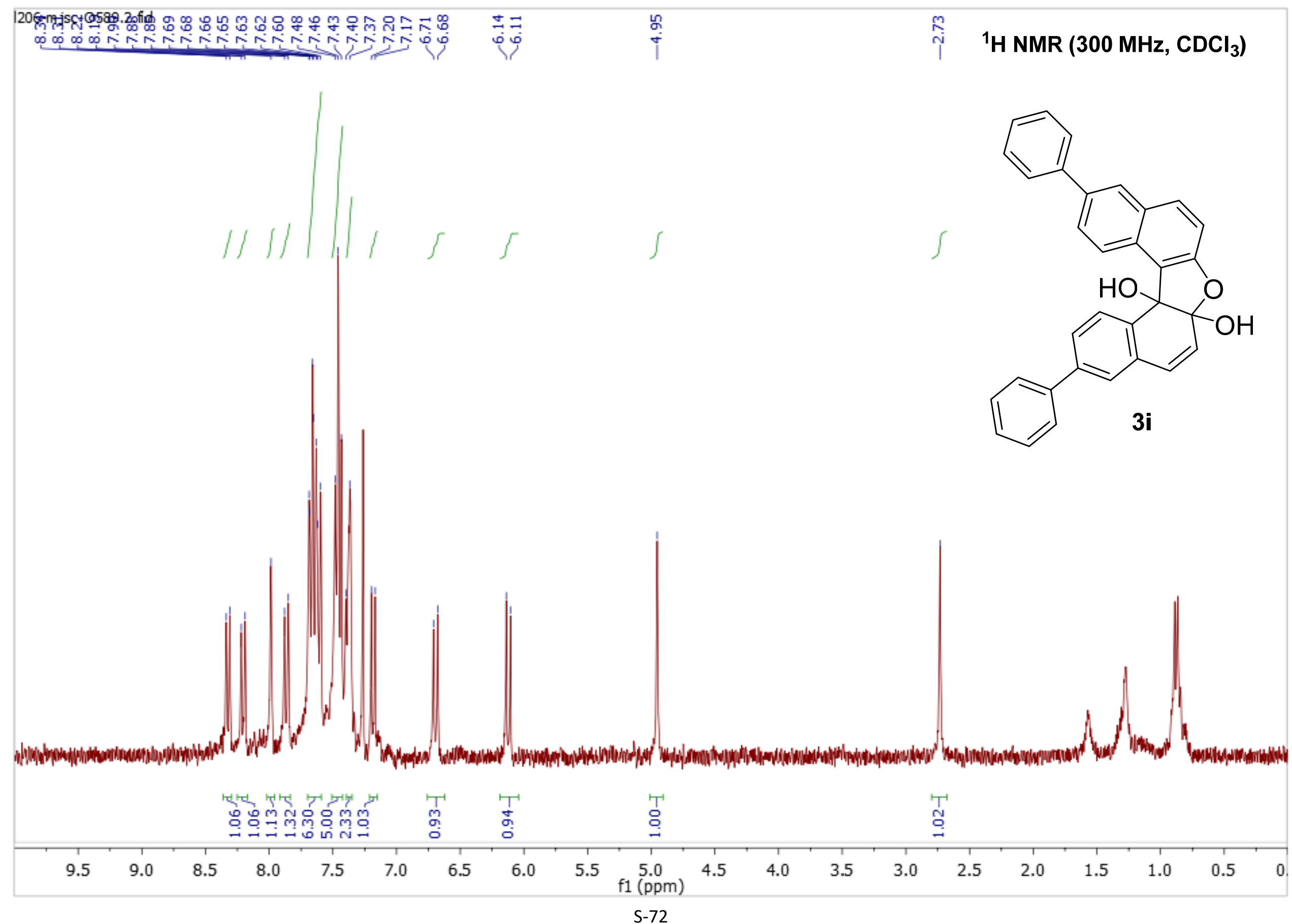




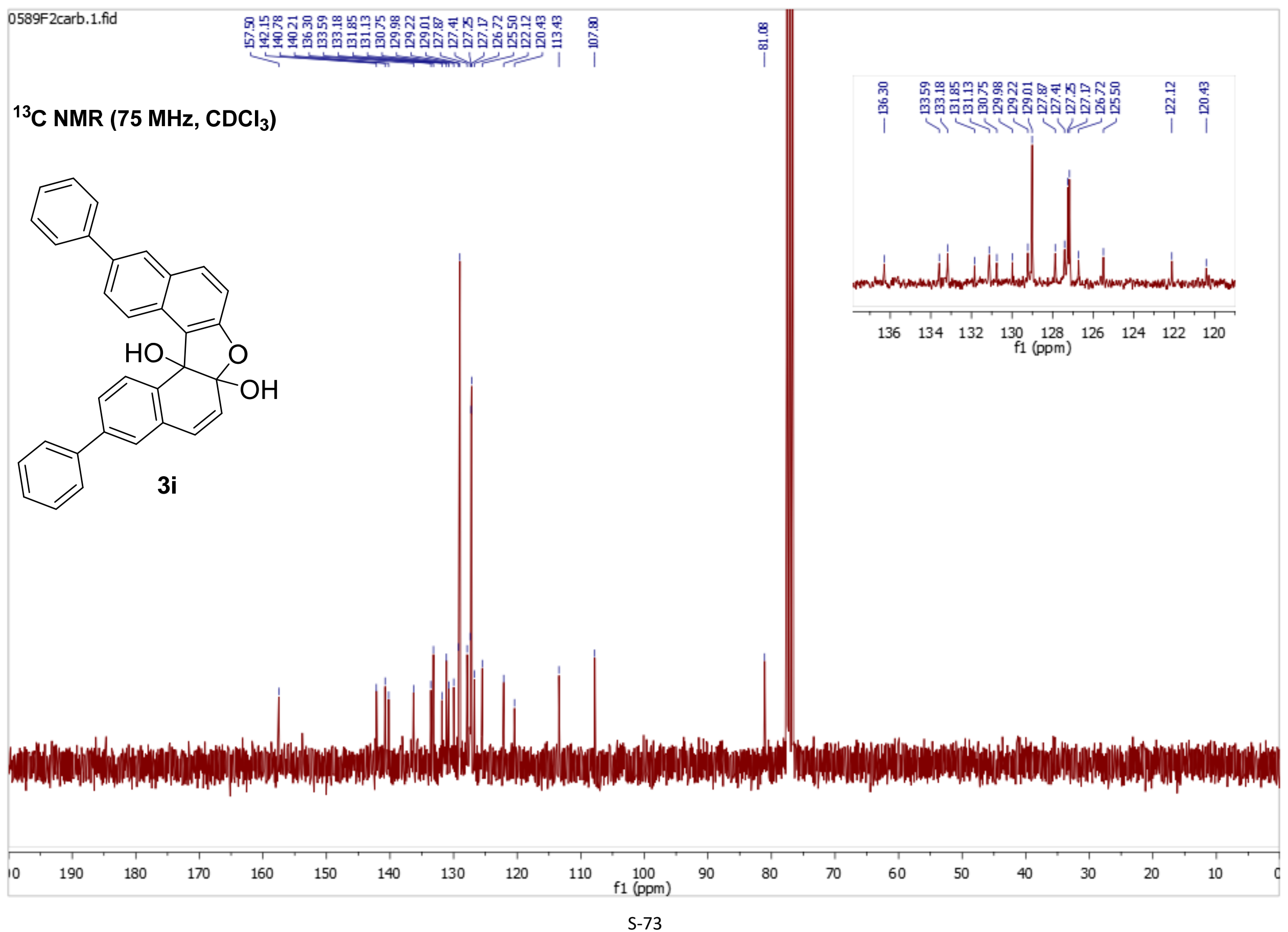




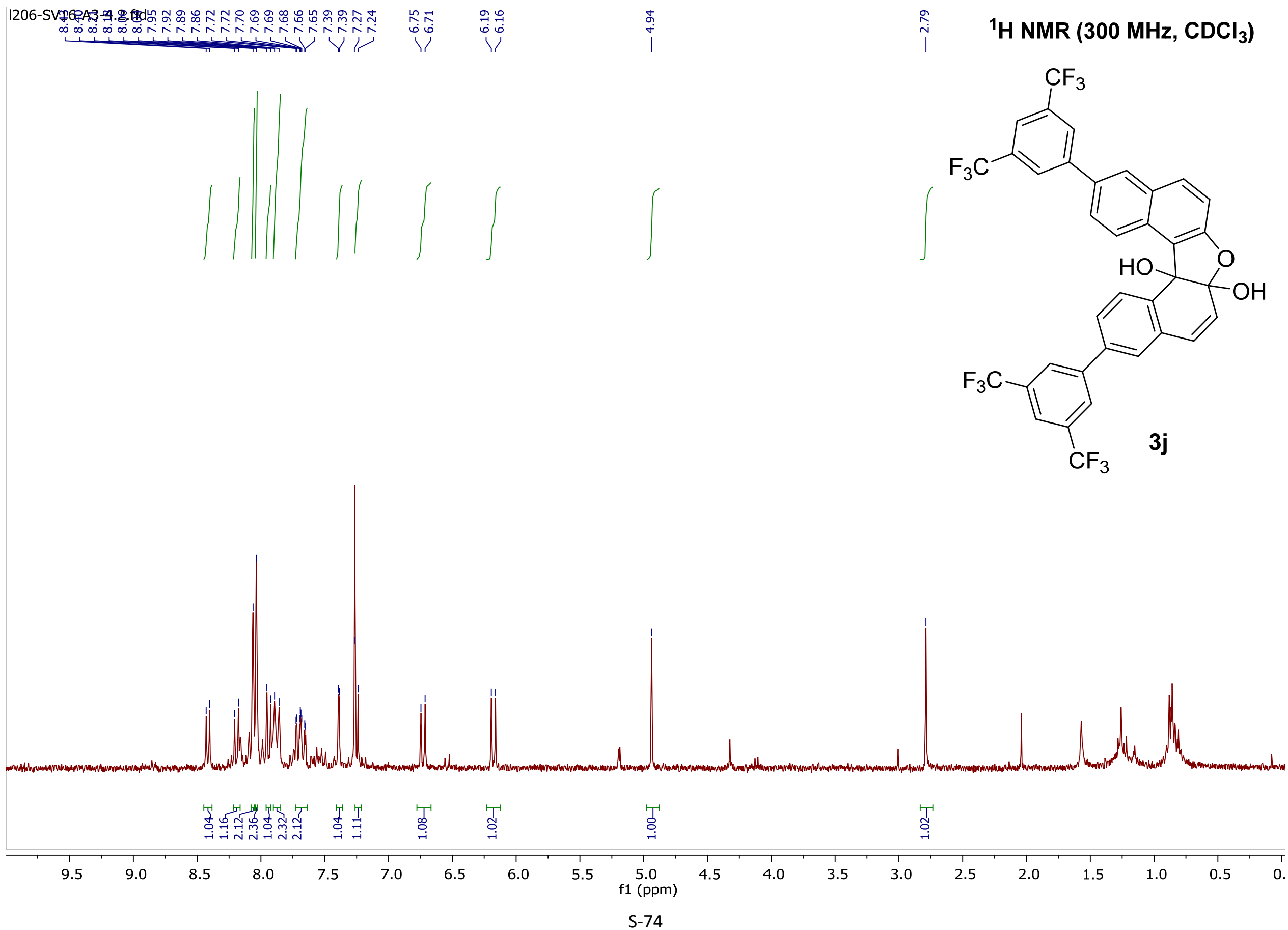




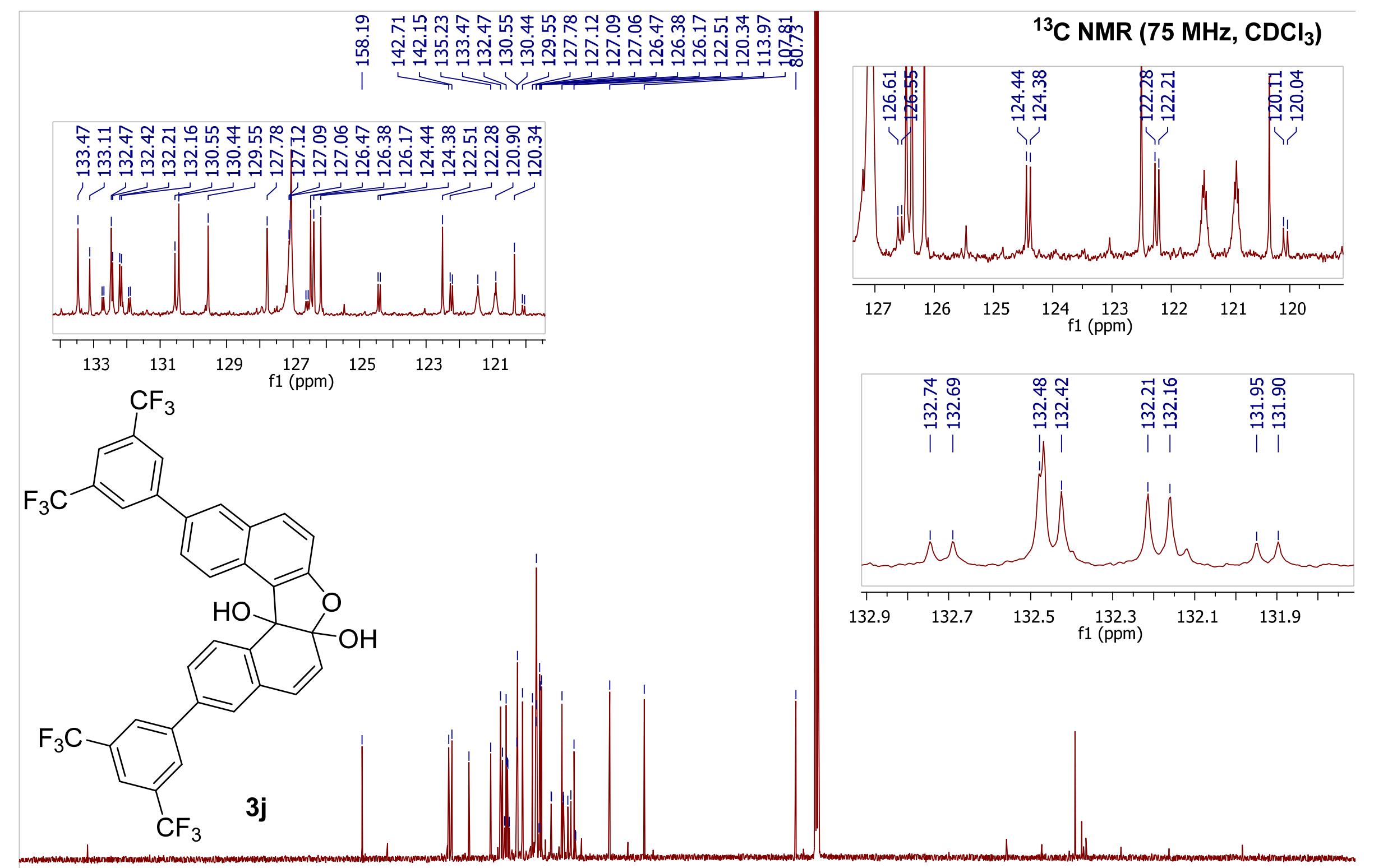

$\begin{array}{llllllllllllllllllllllllllllllll}210 & 200 & 190 & 180 & 170 & 160 & 150 & 140 & 130 & 120 & 110 & \begin{array}{l}100 \\ \mathrm{f} 1(\mathrm{ppm})\end{array} & 90 & 80 & 70 & 60 & 50 & 40 & 30 & 20 & 10 & 0 & -10 & \end{array}$ 
${ }^{19} \mathrm{~F}$ NMR $\left(282 \mathrm{MHz}, \mathrm{CDCl}_{3}\right)$

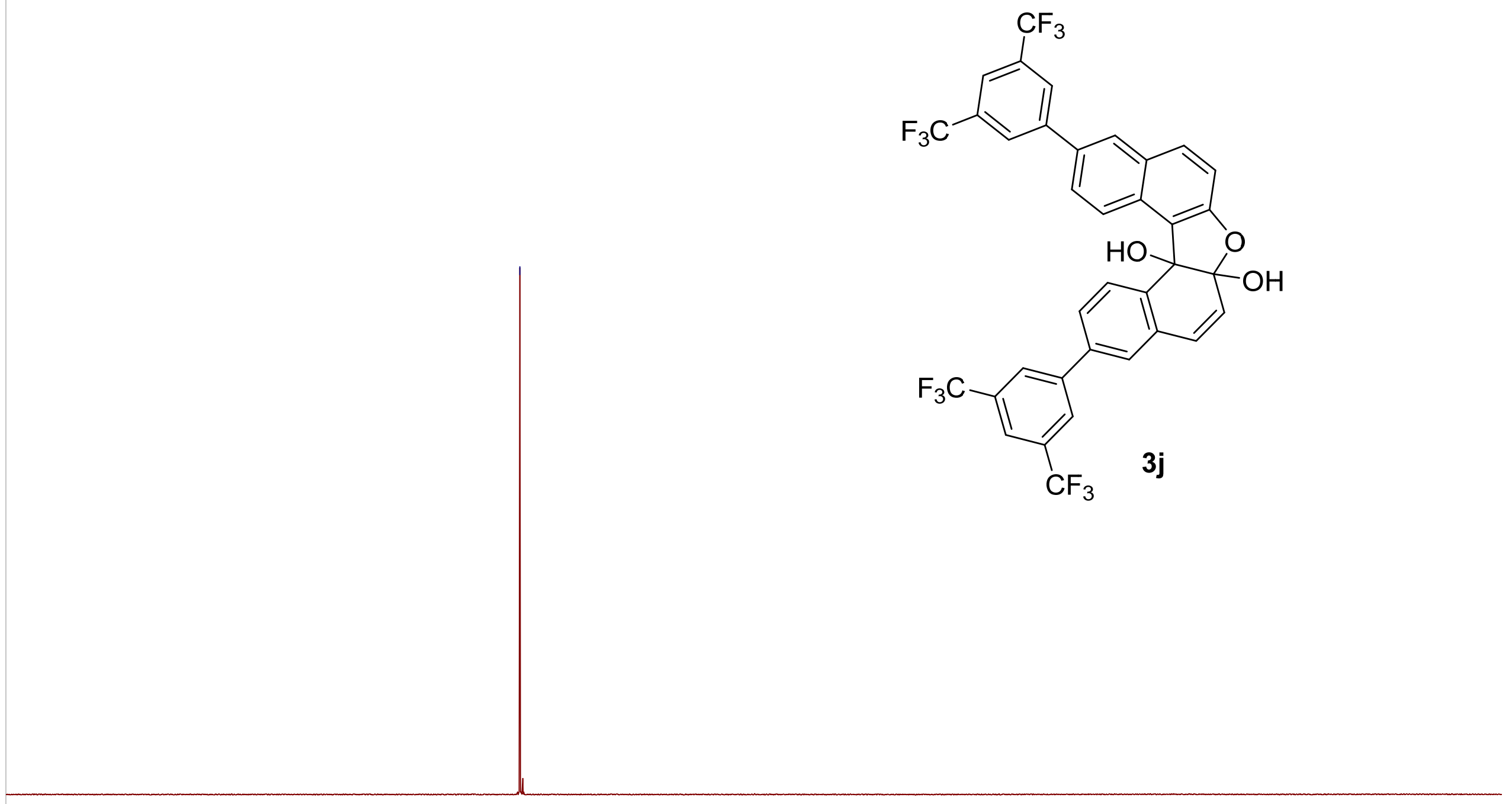

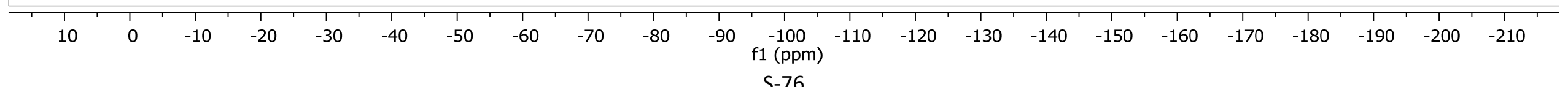




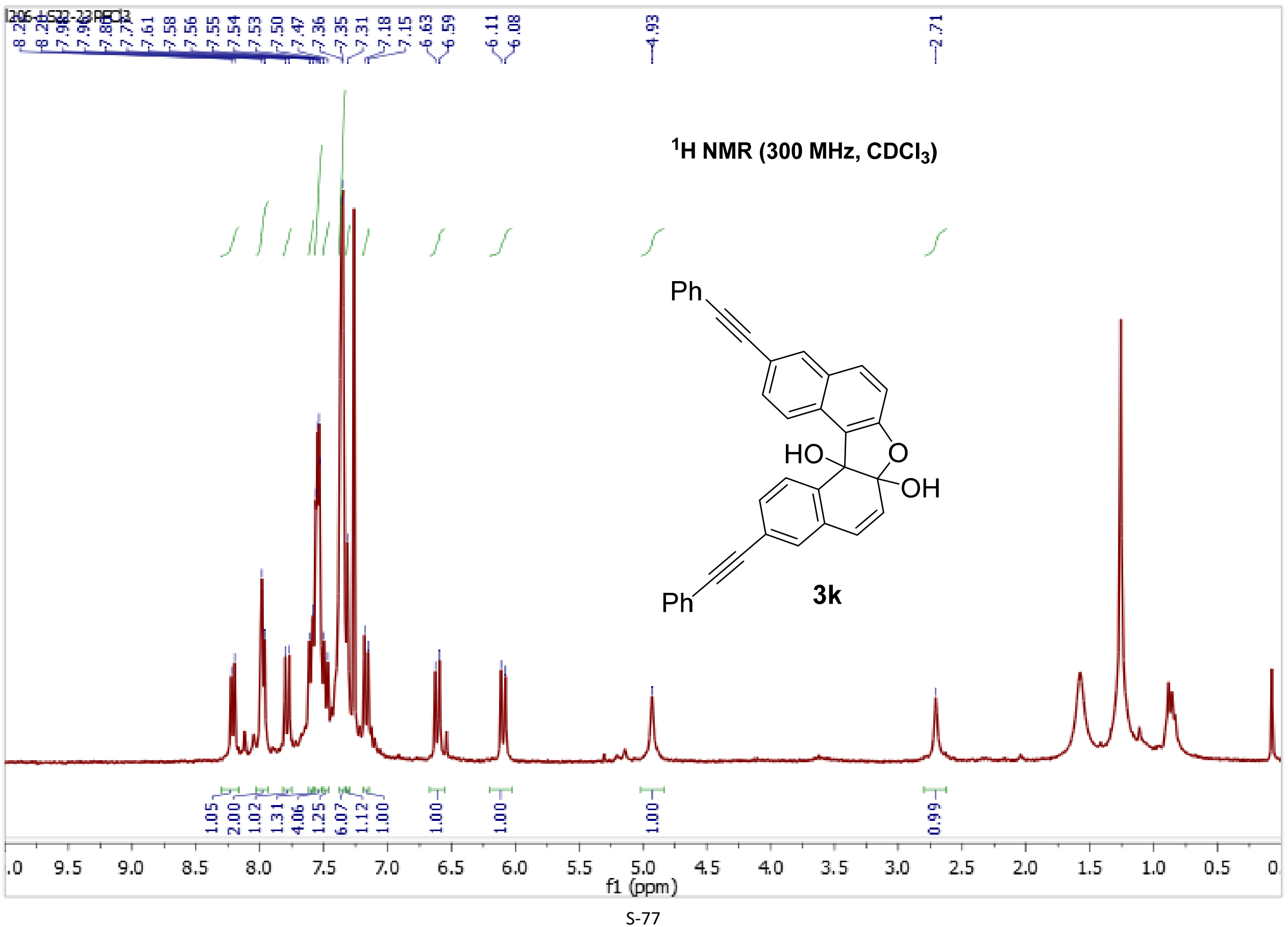




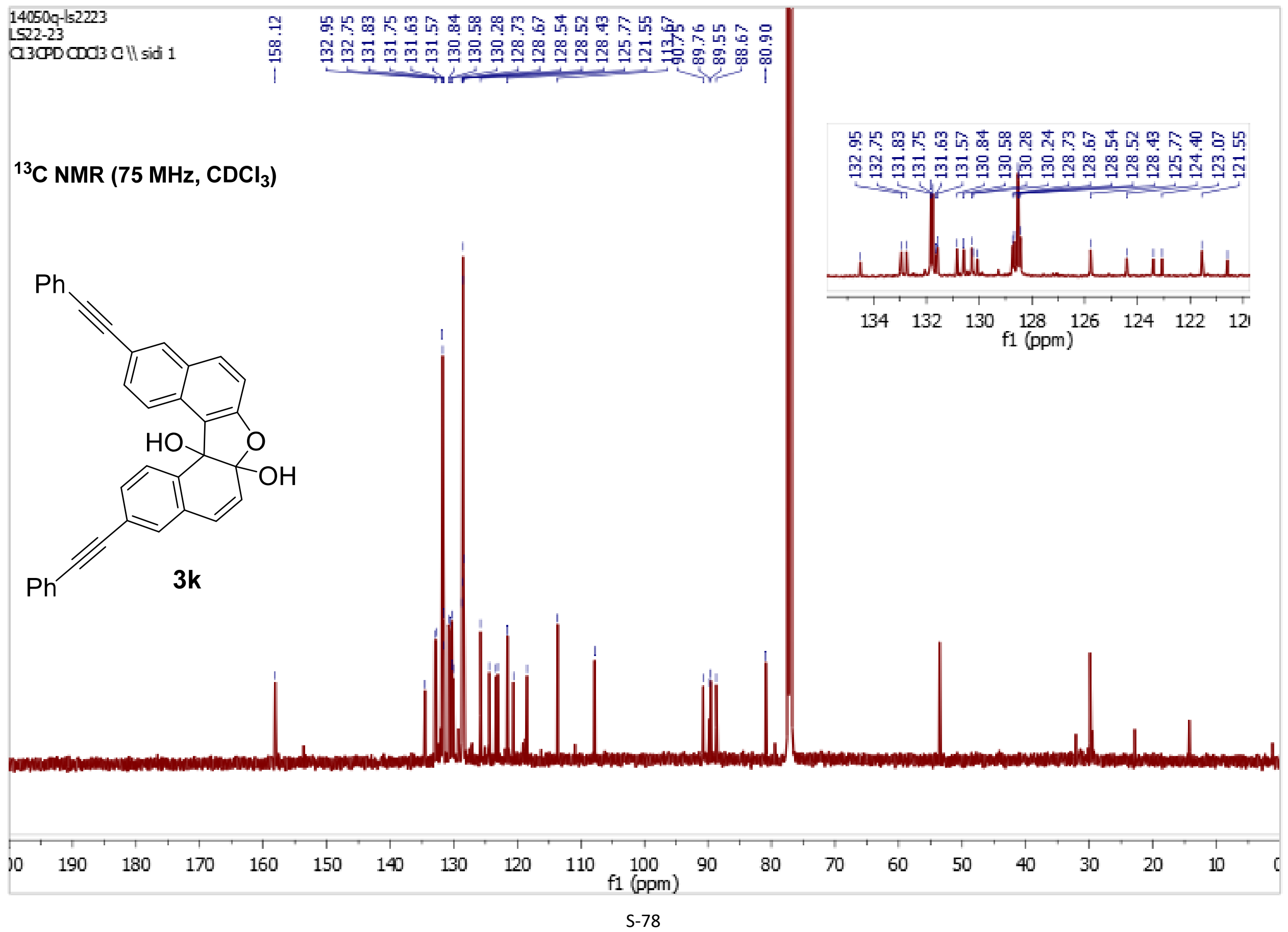




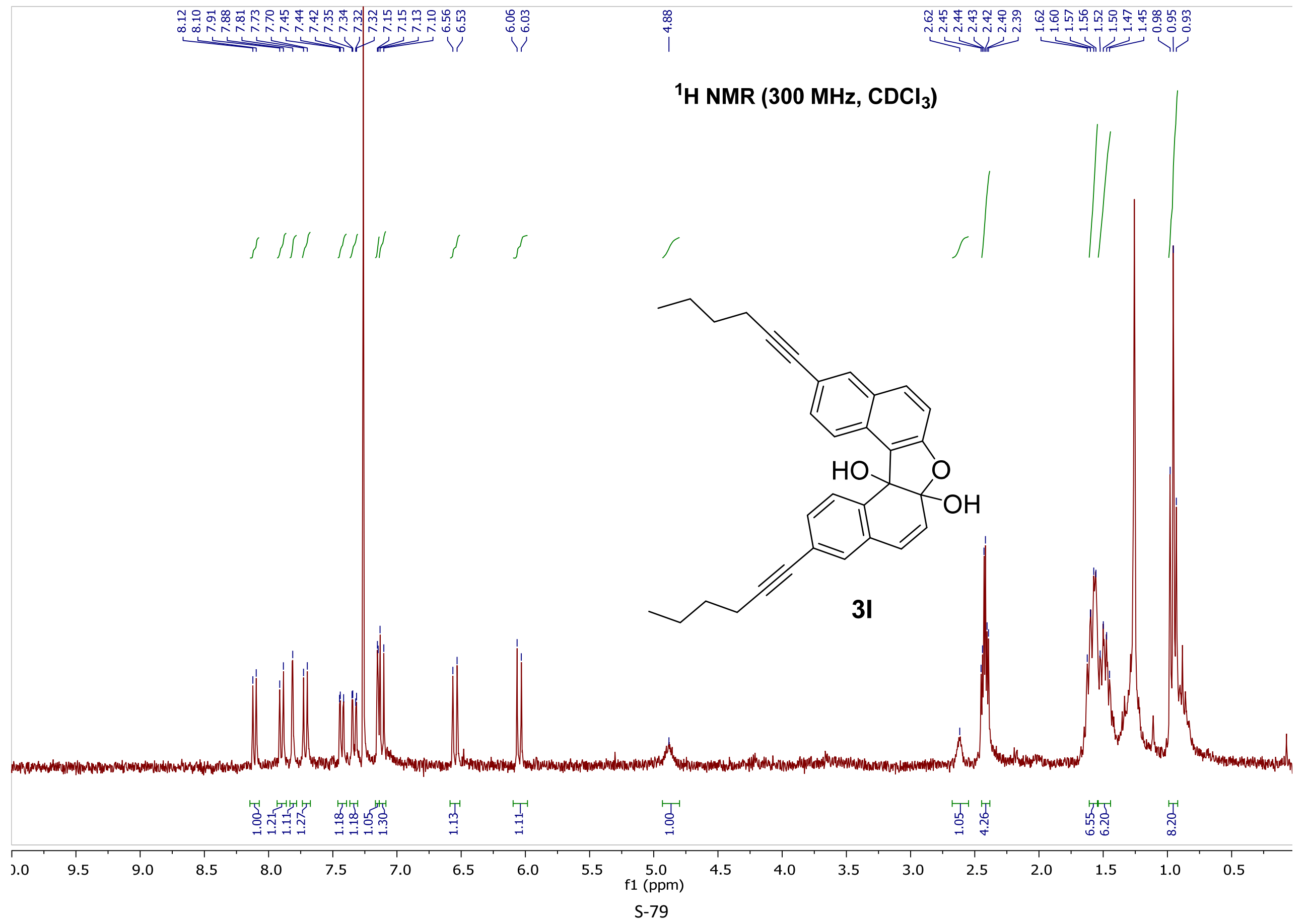




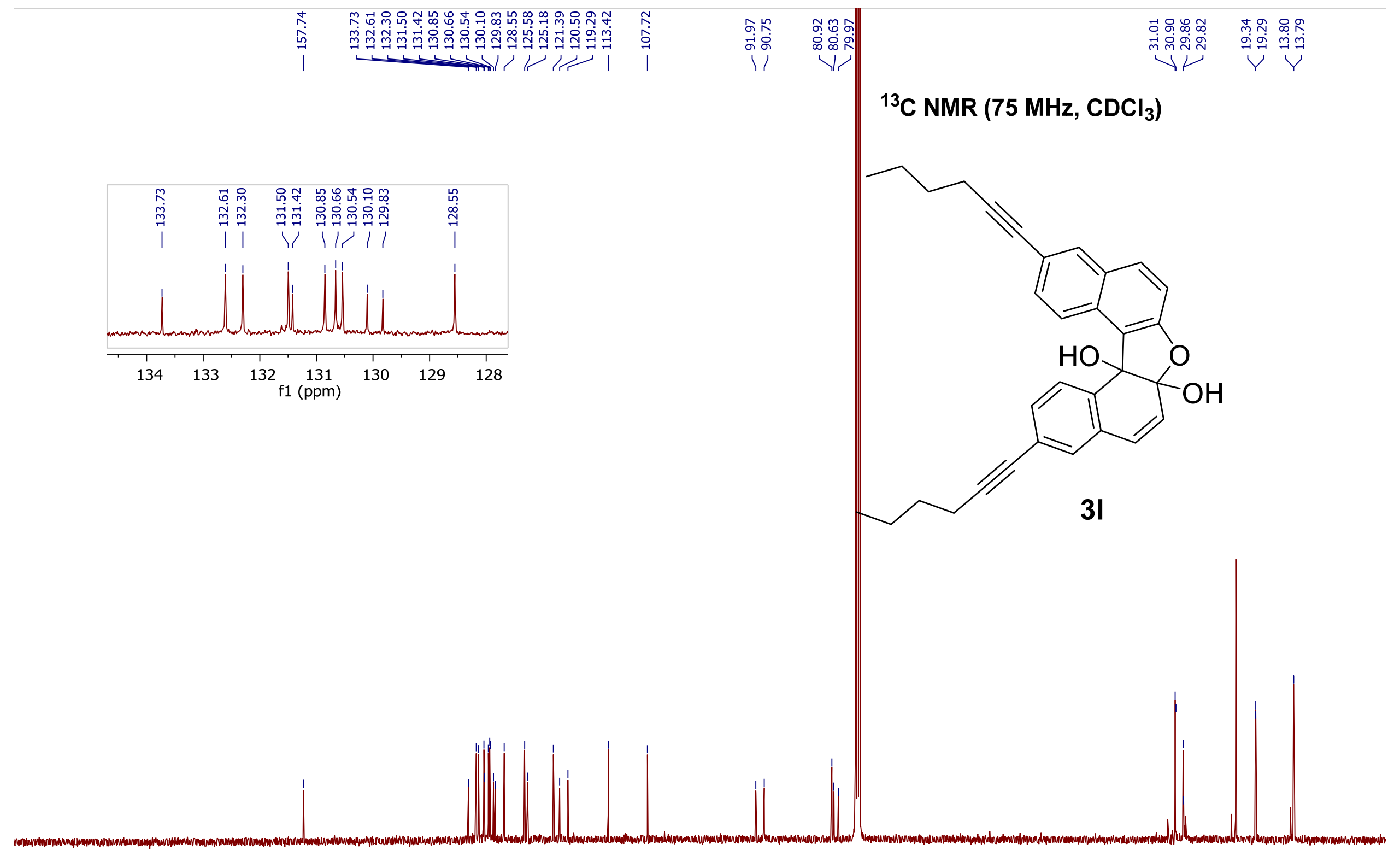

$\begin{array}{llll}190 & 180 & 170 & 160\end{array}$

150

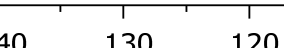

$10 \quad 100$

$90 \quad 80$ $70 \quad 60$

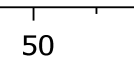

S-80 


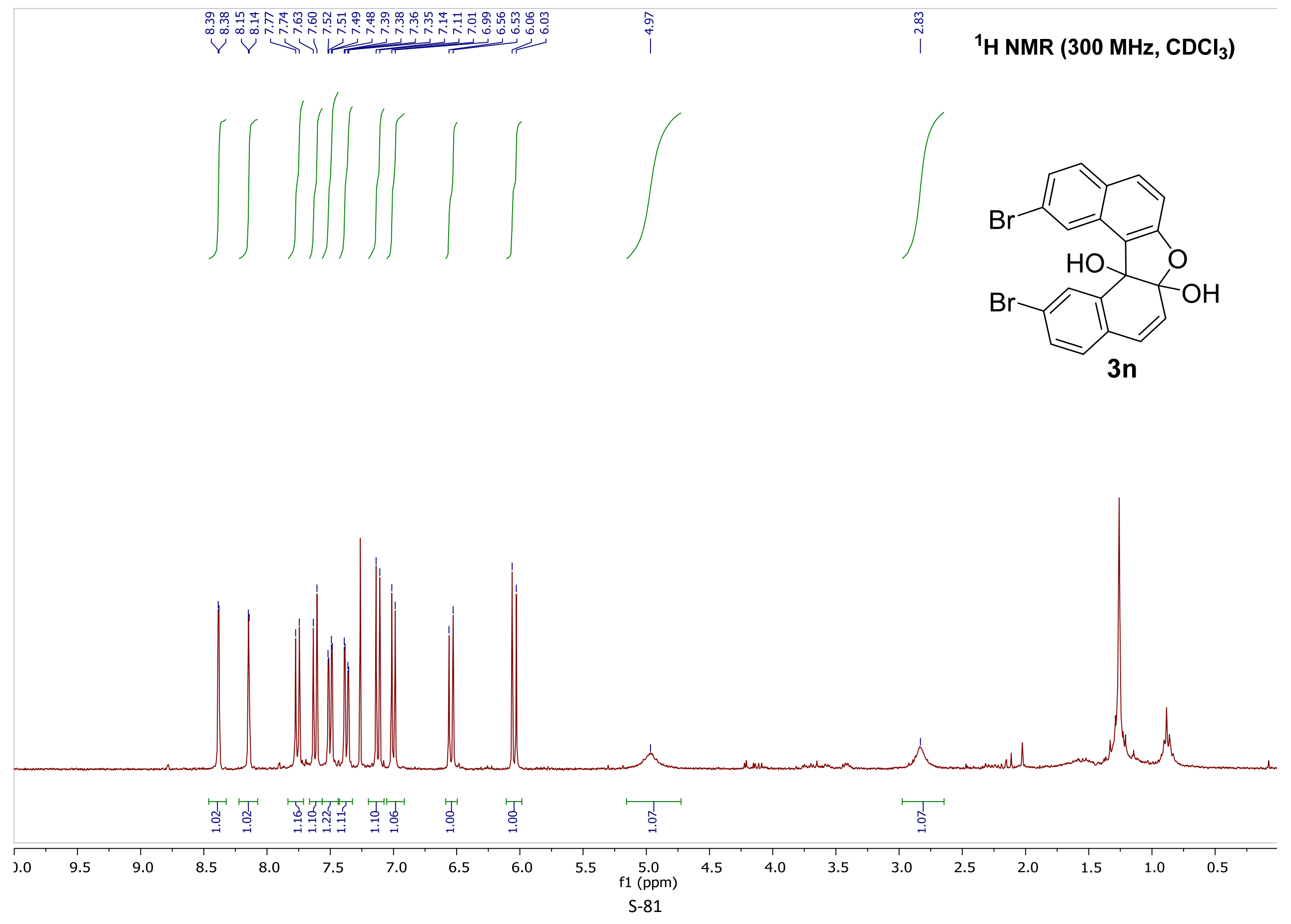



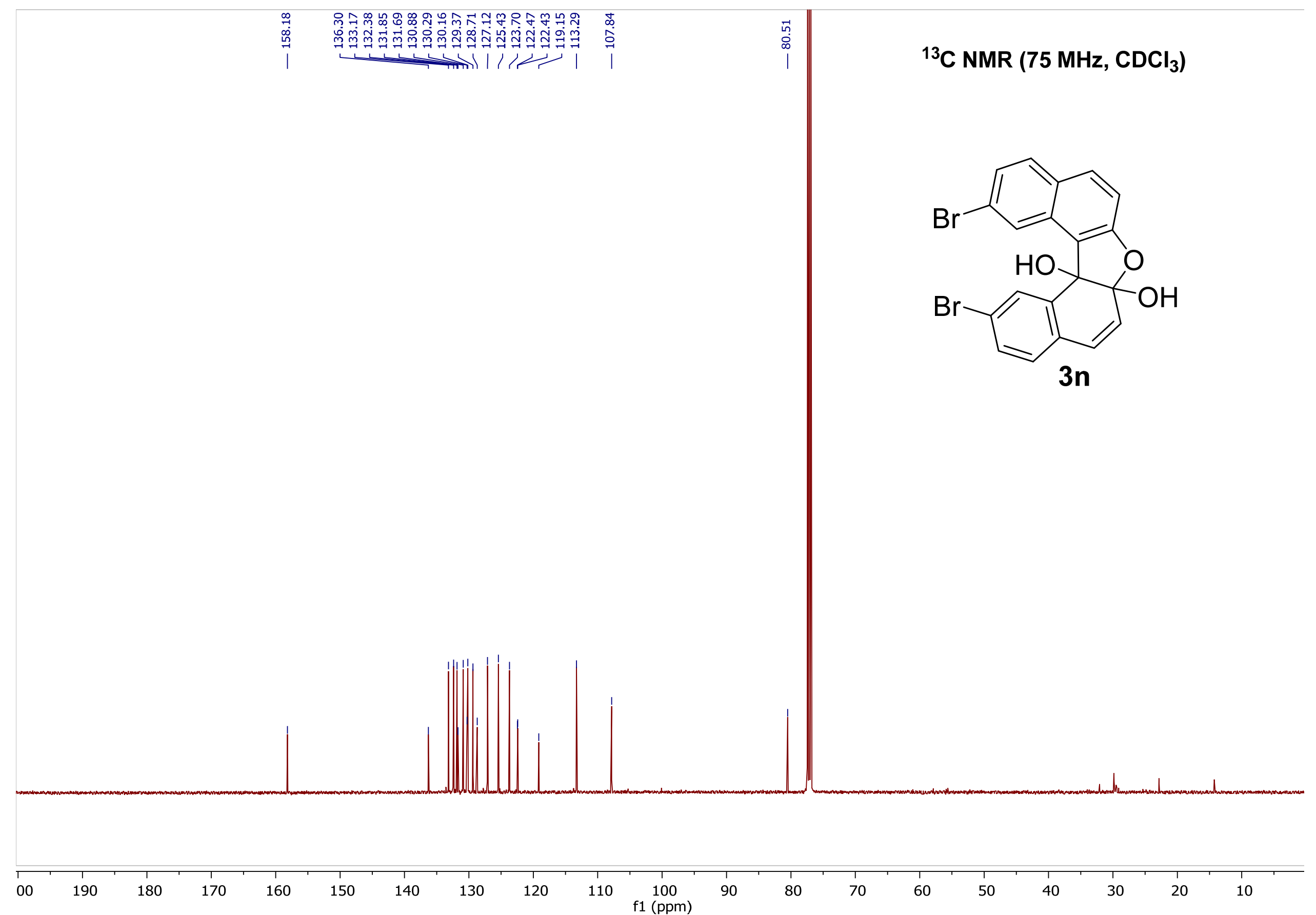


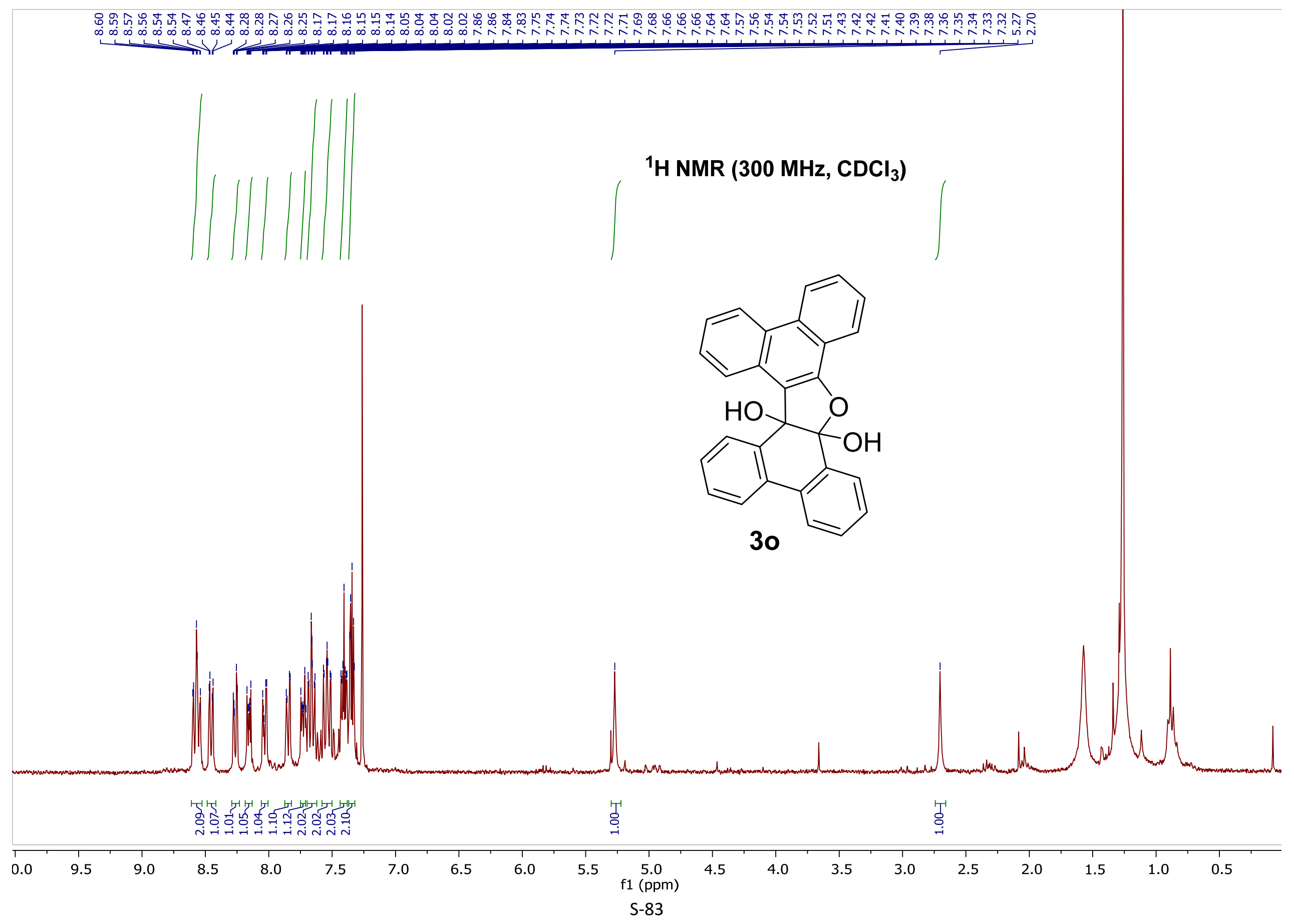




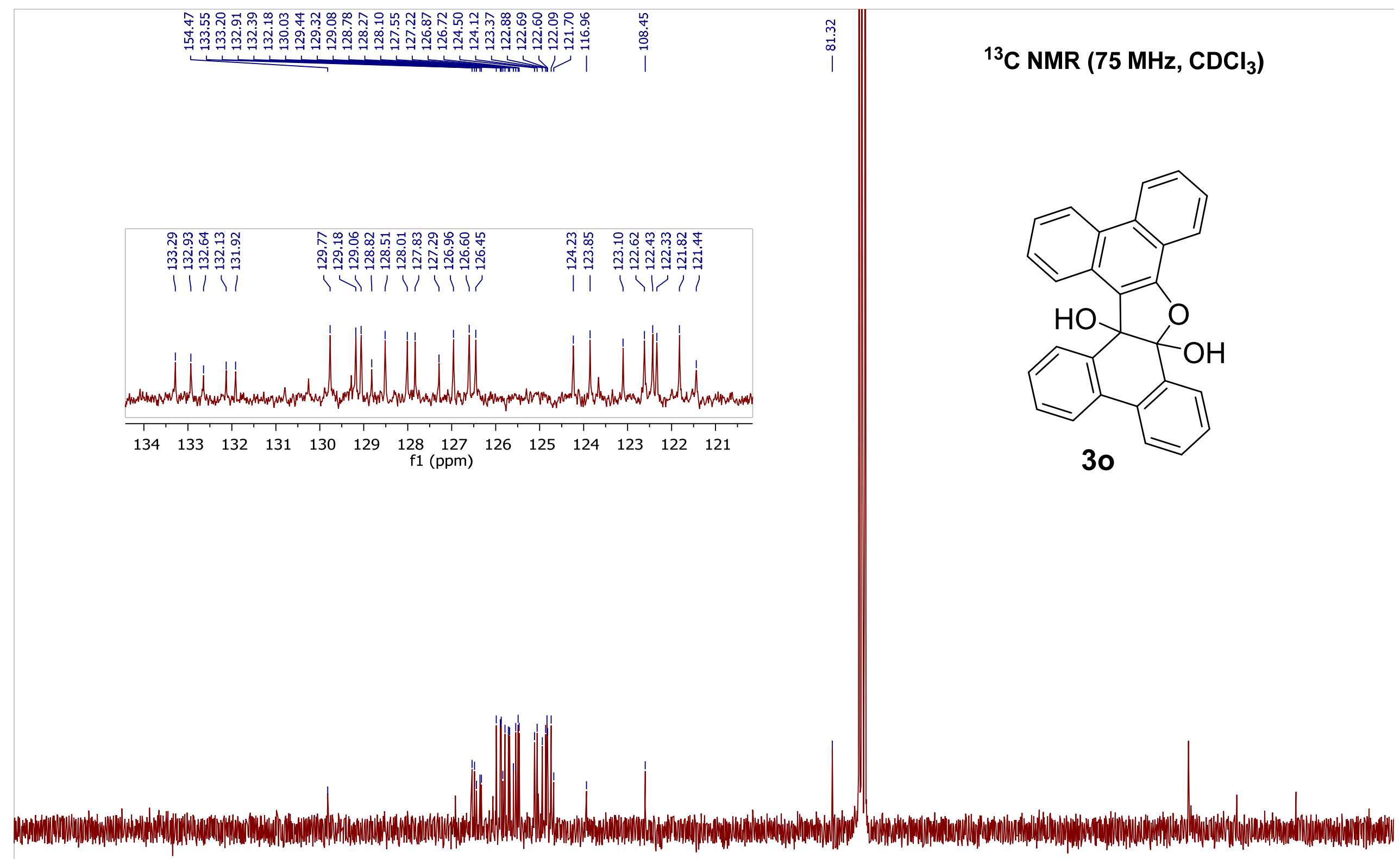

$190 \quad 180$

$170 \quad 160$

150

140

$130 \quad 120$

110

100
$1(\mathrm{ppm})$

$90 \quad 80$

70

60

50

S-84 


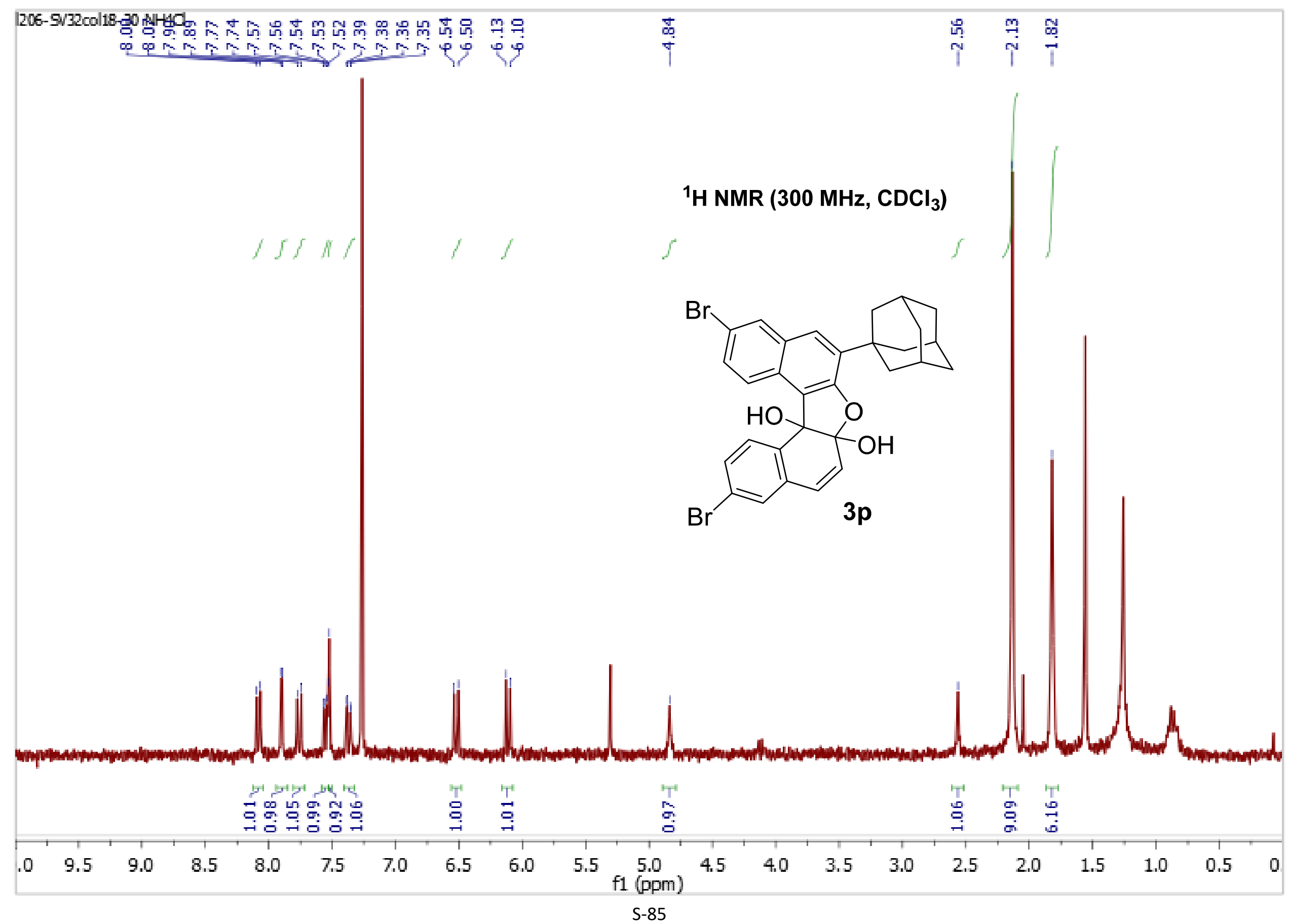




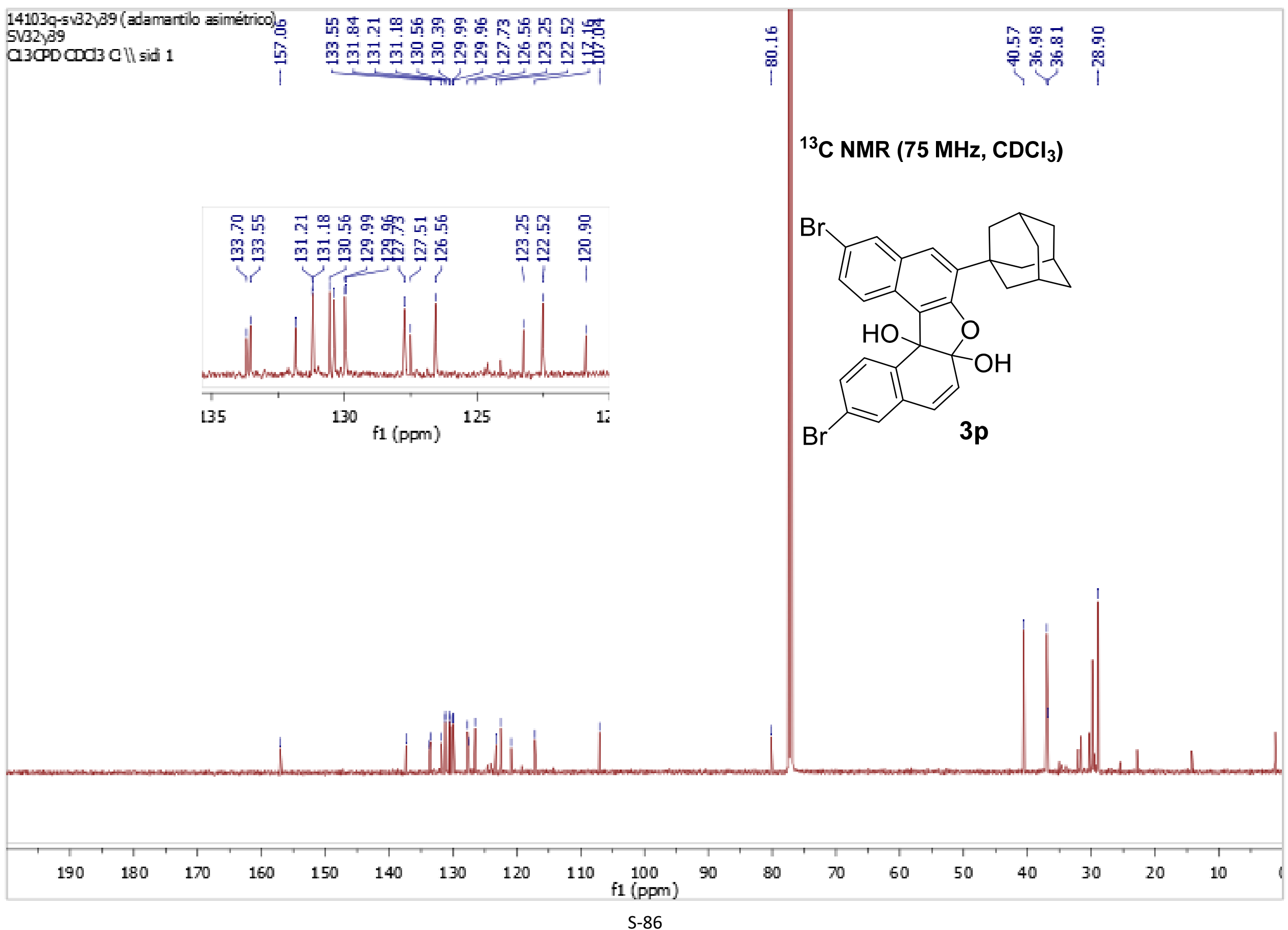

